\title{
Detumbling Space Debris Using Tethers
}

by

Kirk Hovell

A thesis submitted to the Faculty of Graduate and Postdoctoral Affairs in partial fulfillment of the requirements for the degree of

\section{Master of Applied Science in \\ Aerospace Engineering}

\author{
Carleton University \\ Ottawa, Ontario
}

(C) 2017

Kirk Hovell 
This work is dedicated to my late grandmother, Helen Zwicker. 


\begin{abstract}
The population of debris in Earth orbit has exponential growth due to cascaded collisions between orbiting debris. It is critical that high-risk pieces of debris are actively removed to limit the natural growth of the space debris population. A popular strategy to capture space debris is with tethered nets. Detumbling the debris after capture represents one of the outstanding challenges associated with tethered capture of debris.

This thesis presents a new tether configuration that passively enhances the detumbling ability of the tethered net system, and compares it to the presently-accepted tether configuration. A new laboratory facility was developed to allow for an experimental comparison. Both simulation and experimental results agree that the novel tether configuration outperforms the presently-accepted configuration at detumbling debris. The experimental results are the first, to the best of the author's knowledge, to demonstrate the detumbling of debris using tethers.
\end{abstract}




\section{Acknowledgements}

I sincerely thank my supervisor, Steve Ulrich. Without him, I would have never considered pursuing graduate studies, nor would I have fallen in love with research. I also would have missed out on a lot of great squash games! He has encouraged me to step outside my boundaries by presenting my work at several conferences, which has helped me grow as a person. I could not have asked for a better supervisor.

I would also like to thank my family. My mom, Narda, my dad, Jeff, and my sister, Kelly, have all been incredibly supportive and curious throughout my studies. My girlfriend, Molly, has helped me through this incredible journey, with her love, support, and patience.

This research was financially supported in part by the Natural Sciences and Engineering Research Council of Canada under the Canada Graduate Scholarship CGS M-302098-2015 and the Discovery Grant program. In addition, the Ontario Graduate Scholarship, entrance scholarships to the Mechanical and Aerospace Engineering department, and Teaching Assistantships helped fund my research. 


\section{Table of Contents}

Abstract $\quad$ iii

Acknowledgements $\quad$ iv

List of Tables $\quad$ vi

List of Figures $\quad$ vii

List of Symbols viii

Chapter 1 Introduction 1

1.1 Motivation . . . . . . . . . . . . . . . . . . . 1

1.2 Problem Statement . . . . . . . . . . . . . . . . . 1

1.3 Previous Work . . . . . . . . . . . . . . . . . . . 2

1.4 Thesis Objectives . . . . . . . . . . . . . . 5

1.5 Contributions ....................... 6

1.6 Organization ......................... 7

$\begin{array}{lll}\text { Chapter } 2 & \text { On-Orbit Detumbling } & 8\end{array}$

2.1 Introduction . . . . . . . . . . . . . . . . 8

2.2 On-orbit Dynamics . . . . . . . . . . . . . . . . 8

2.2.1 Dynamics Formulation - Sub-Tether Configuration . . . . . . . 9

2.2.2 Attitude Motion . . . . . . . . . . . . . . . 10

2.2.3 Translational Motion . . . . . . . . . . . . . . . 11

2.2.4 Equations of Motion . . . . . . . . . . . . . . 13

2.2.5 Dynamics Formulation - Single Tether Configuration . . . . . 14

2.3 On-Orbit Numerical Simulations . . . . . . . . . . . . . . . . . 14

2.3.1 Case $1 \ldots \ldots \ldots \ldots$. . . . . . . . . . . . . . 18

2.3 .2 Case $2 \ldots \ldots . \ldots \ldots 20 . \ldots \ldots$ 
2.3 .3 Case $3 \ldots \ldots \ldots \ldots \ldots \ldots$

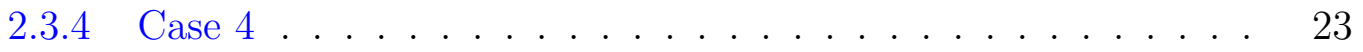

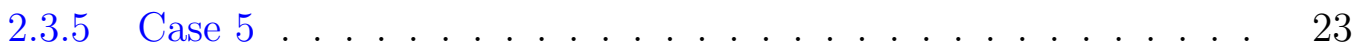

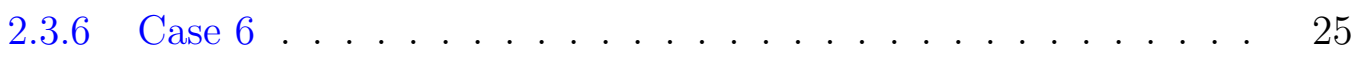

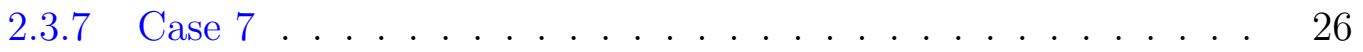



$\begin{array}{lll}\text { Chapter } 3 & \text { Experimental Facility } & 30\end{array}$

3.1 Introduction . . . . . . . . . . . . . . . . . . . 30

3.2 Laboratory Contributions . . . . . . . . . . . . . . . 33

3.2 .1 Floatation System _ . . . . . . . . . . . . . . . 33

3.2 .2 Propulsion System . . . . . . . . . . . . . . . . . . 35

3.2 .3 Air Refill Station . . . . . . . . . . . . . . . . . . . 39

3.2 .4 Reaction Wheel . . . . . . . . . . . . . . . . . . . . . 39

3.2.5 Power and Computer System _ . . . . . . . . . . . 42

3.2 .6 Emergency Stop System . . . . . . . . . . . . . . . 42

3.2 .7 Ground Truth System . . . . . . . . . . . . . . . . 45

3.2 .8 Software . . . . . . . . . . . . . . . . . 45

3.3 Experimental Setup . . . . . . . . . . . . . . . . . . . . . 47

3.3 .1 Tether Model . . . . . . . . . . . . . . . . . . . . . . . 47

3.3 .2 Controller Design . . . . . . . . . . . . . . . . . . . . 48

Chapter $4 \quad$ Planar Detumbling $\quad 52$

4.1 Introduction . . . . . . . . . . . . . . . . . . . 52

4.2 Planar Dynamics . . . . . . . . . . . . . . . . . . . . 53

4.2.1 Translational Motion . . . . . . . . . . . . . . 55

4.2 .2 Rotational Motion . . . . . . . . . . . . . . . 55

4.2.3 Single Tether Equations of Motion _. . . . . . . . . . 56

4.2.4 Sub-Tether Equations of Motion . . . . . . . . . . . 58

4.3 Numerical Simulations . . . . . . . . . . . . . . . . . . . . . 61

4.3 .1 Thrust Stabilization _. . . . . . . . . . . . . . 63 
4.3.2 TSS Spin Stabilization . . . . . . . . . . . . . . 66

$\begin{array}{lll}\text { Chapter } 5 & \text { Experimental Validation } & 72\end{array}$

5.1 Introduction . . . . . . . . . . . . . . . . . . . . . . . . . 72

5.2 Single Tether Results . . . . . . . . . . . . . . . . . . . 73

5.3 Sub-tether Results . . . . . . . . . . . . . . . . . . . 75

$\begin{array}{lll}\text { Chapter } 6 & \text { Conclusion } & 78\end{array}$

6.1 Thesis Summary . . . . . . . . . . . . . . . . . . . . 78

6.2 Significance of Work . . . . . . . . . . . . . . . . . . . 80

6.3 A Note on Practicality . . . . . . . . . . . . . . . . . 80

6.4 Recommendations for Future Work . . . . . . . . . . . . . . 81

$\begin{array}{ll}\text { Bibliography } & 83\end{array}$

$\begin{array}{lll}\text { Appendix A } & \text { Laboratory Contributions } & 88\end{array}$

A.1 Floatation System . . . . . . . . . . . . . . . . . . 88

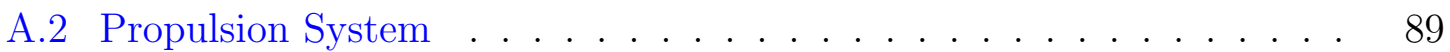

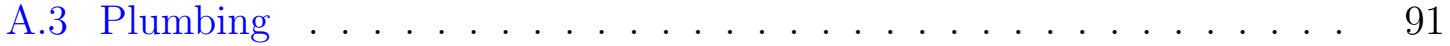

A.3.1 Using PVC Fittings . . . . . . . . . . . . . . . . . . . . . 93

A.4 Solenoid Valve Actuation . . . . . . . . . . . . . . . . 95

A.5 Air Refill System . . . . . . . . . . . . . . . . . 98

A.6 Reaction Wheel . . . . . . . . . . . . . . . . . . . 100

A.7 Power System . . . . . . . . . . . . . . . . . . . . . . . . 103

A.8 Emergency Stop System _ . . . . . . . . . . . . . . . . . 107

A.9 Ground Truth System . . . . . . . . . . . . . . . . . . . . . . 109

A.9.1 Calibration . . . . . . . . . . . . . . . . . 112

A.9.2 Alignment . . . . . . . . . . . . . . . . . . 113

A.10 Software . . . . . . . . . . . . . . . . . . 115 


\section{List of Tables}

$2.1 \quad$ Orbital elements used to define initial orbit of TSS. . . . . . . 16

2.2 Initial conditions and TSS parameters for simulations. . . . . . 17

4.1 Initial conditions and TSS parameters for planar simulations and experiments. .................... 62

4.2 Initial conditions for planar thrust stabilization simulations and experiments. .................. 63

4.3 Initial conditions for TSS spin planar simulations and experiments. 68

A.1 Part list for the floatation system. . . . . . . . . . 88

A.2 Part list for the propulsion system. . . . . . . . . . . . 90

A.3 Part list for the plumbing system. . . . . . . . . . . . . . 92

A.4 Part list for the transistor board. . . . . . . . . . . . . . 97

A.5 Desired and achieved air quality. . . . . . . . . . . . 99

A.6 Part list for the air refill system. . . . . . . . . . . . . . 99

A.7 Part list for the reaction wheel. . . . . . . . . . . . . 100

A.8 Part list for the power system. . . . . . . . . . . . 105

A.9 Part list for the emergency stop system. . . . . . . . . . . . 107

A.10 Phasespace platform software ID numbers. . . . . . . . . . . 111 


\section{List of Figures}

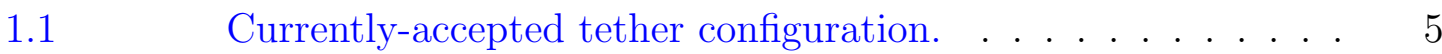

$2.1 \quad$ Reference frame and vector definition for dynamics modeling. . 9

2.2 Simple spring-damper system, where two masses are attached via a spring and damper in parallel. . . . . . . . . . . . . . 12

2.3 Angle $\phi$ represents the current angle between the tether and its equilibrium point. Reference frame $\mathcal{F}_{B}$ defines the body-fixed reference frame on the target debris. . . . . . . . . . . 18

2.4 Resulting on-orbit TSS motion for Case 1. Dashed lines represent the angular rate about the $\vec{B}_{y}$ axis, and the dotted and solid lines represent the angular rate about the $\vec{B}_{x}$ and $\vec{B}_{z}$ axes, respectively, as shown in Fig. 2.3. . . . . . . . . . . . . 19

$2.5 \quad$ Resulting on-orbit TSS motion for Case $2 \ldots \ldots \ldots . \ldots 21$

$2.6 \quad$ Resulting on-orbit TSS motion for Case $3 \ldots \ldots \ldots$

$2.7 \quad$ Resulting on-orbit TSS motion for Case $4 \ldots \ldots \ldots$

$2.8 \quad$ Resulting on-orbit TSS motion for Case $5 \ldots \ldots \ldots$

$2.9 \quad$ Resulting on-orbit TSS motion for Case $6 \ldots \ldots \ldots 27$

$2.10 \quad$ Resulting on-orbit TSS motion for Case $7 \ldots \ldots \ldots$

3.1 Overview of the Spacecraft Proximity Operations Testbed. . . 32

$3.2 \quad$ Spacecraft platforms built for the SPOT. . . . . . . 33

3.3 Bottom of air bearing is shown on the left. The top of the bearing and mounting holes are shown on the right. . . . . . .

3.4 Air flow diagram responsible for the on board propulsion and floatation systems. Only two thrusters are shown for clarity. . 34

3.5 Air flow on the top side of the bus deck. Here, the air passes through regulators before being divided to the thrusters and the air bearings. . . . . . . . . . . . . . . . 36 
3.6 Air flow on the underside of the bus deck. Here, the air is delivered to the 8 thrusters and 3 air bearings. . . . . . . . 37

3.7 Thruster numbers and body-fixed reference frame on each spacecraft platform. . . . . . . . . . . . . . . 38

3.8 Air refill station. . . . . . . . . . . . . . . . . 40

3.9 Reaction wheel. . . . . . . . . . . . . . . 41

3.10 Electrical schematic present on the avionics deck of the spacecraft platforms. . . . . . . . . . . . . . . . . 43

3.11 Avionics deck on board the spacecraft platforms. This deck contains the power subsystem, the on board computer, the reaction wheel, and the control panel. . . . . . . . . . 44

3.12 Experimental non-linear tether stiffness model, generated by hanging known masses from each tether and measuring the resulting stretch. . . . . . . . . . . . . . . . 48

3.13 RED chaser tethered to the uncooperative BLACK debris on the granite table in Carleton's SPOT. . . . . . . . . . . . 49

3.14 Block diagram of the PD controller for translational control, where $\mathbf{r}_{\text {des }}(t)$ is the desired position and $\mathbf{r}_{c}(t)$ is the actual position. 49

3.15 Block diagram of the PD controller for rotational control, where $\theta_{\text {des }}(t)$ is the desired angle and $\theta_{c}(t)$ is the actual angle. . . . 50

4.1 Reference frame and vector definition for planar dynamics modeling. . . . . . . . . . . . . . . . 53

4.2 Simple spring-damper system, where two masses are attached via a spring and damper in parallel. . . . . . . . . . . 56

4.3 Positive angle $\phi$ represents the current angle between $\vec{T}_{x}$ and the tether. The sub-tether configuration uses an imaginary single tether to calculate the tether angle. . . . . . . . . .

4.4 Resulting simulated TSS motion for planar thrust stabilization, comparing the two tether configurations. . . . . . . . . 64 
4.5 Snapshots of an animation of the resulting motion for the thrust stabilization simulation. The red spacecraft is the chaser and the black is the target. The single tether is shown in gray. . .

4.6 Snapshots of an animation of the resulting motion for the thrust stabilization simulation. The red spacecraft is the chaser and the black is the target. The sub-tethers are shown in gray. . .

4.7 Resulting simulated motion for TSS spin stabilization, comparing the two tether configurations. . . . . . . . . . . . . 69

4.8 Snapshots of an animation of the resulting motion for the TSS spin stabilization simulation. The red spacecraft is the chaser and the black is the target. The single tether is shown in gray.

4.9 Snapshots of an animation of the resulting motion for the TSS spin stabilization simulation. The red spacecraft is the chaser and the black is the target. The sub-tethers are shown in gray.

$5.1 \quad$ Two spacecraft platforms tethered together on the planar gravityoffset testbed at Carleton's SPOT. . . . . . . . . . . . 73

5.2 Comparing experimental and simulated response for the planar TSS spin stabilization technique with a single tether. . . . . . 74

5.3 Comparing experimental and simulated response for the planar TSS spin stabilization technique with sub-tethers. . . . . . . 76

5.4 Comparing planar experimental results of the two tether configurations when a TSS spin is used to regulate a tumbling target. 77

A.1 Air bearing mounted to the spacecraft platform. The blue air bearing is fed air through the flexible clear hose, which connects to the bearing through the Elbow Air Fitting. A mounting screw attaches the bearing to the base of the platform. The mounting clip secures the mounting screw to the bearing. . . . 
A.2 Bottom of air bearing is shown on the left. The top and mounting clip holes are shown on the right. The spherical groove is where the mounting screw sits. . . . . . . . . . . . . 89

A.3 Solenoid valve attached to air nozzle. . . . . . . . . . 90

A.4 Air nozzle holes in the spacecraft structure. . . . . . . . . 91

A.5 Air flow on the top side of the bus deck. . . . . . . . 93

A.6 Air flow on the underside of the bus deck. . . . . . . . . 94

A.7 A barbed fitting for use with the PVC tubing. . . . . . . . 94

A.8 The process of attaching PVC tubing to a fitting. . . . . 95

A.9 Air flow diagram responsible for the on board propulsion and floatation systems. . . . . . . . . . . . . . 96

A.10 The board of MOSFETs used to control the solenoid valves. . 97

A.11 Motor speed vs available torque for the reaction wheel motor when the supply voltage is capped at 12 V . . . . . . . . . . 103

A.12 Avionics deck on board the spacecraft platforms. This deck contains the power subsystem, the on board computer, the reaction wheel, and the control panel. . . . . . . . . . . . . 104

A.13 Electrical schematic present on the spacecraft platforms. . . 106

A.14 Control panel on the avionics deck of the spacecraft platform. It includes the main power switch, a charging port, the Emergency Stop LED, and the battery monitoring LED. . . . . . . . . 108

A.15 Camera positioning to ensure even coverage across the table. The black circles represent the cameras, and the red cones represent the field of view of each camera. . . . . . . . . . . . 109

A.16 Overview of the Spacecraft Robotics and Control Laboratory. The black PhaseSpace cameras can be seen surrounding the lab. 110 A.17 PhaseSpace Microdriver controlling the tracking LEDs. . . . 110 A.18 Wand used for calibrating and aligning the PhaseSpace system. 113 


\section{List of Symbols}

\begin{tabular}{|c|c|}
\hline A & Attitude matrix \\
\hline $\overrightarrow{\mathcal{F}}_{x}$ & Vectrix of reference frame $x$ \\
\hline$\tau$ & Torque, $\mathrm{N} \cdot \mathrm{m}$ \\
\hline$F$ & Force magnitude, $\mathrm{N}$ \\
\hline $\mathbf{F}$ & Force vector components, $\mathrm{N}$ \\
\hline$m$ & Mass, kg \\
\hline$J$ & Moment of inertia, $\mathrm{kg} \cdot \mathrm{m}^{2}$ \\
\hline$\mu$ & Gravitational parameter of the Earth, $\mathrm{m}^{3} / \mathrm{s}^{2}$ \\
\hline $\mathbf{L}$ & Tether vector components, m \\
\hline$L_{0}$ & Tether unstretched length, m \\
\hline$\Delta L$ & Tether stretch magnitude, m \\
\hline $\mathbf{a}_{x}$ & Tether attachment point on body $x$ with respect to centre of mass, $\mathrm{m}$ \\
\hline $\mathbf{a}_{i}$ & $i^{\text {th }}$ sub-tether attachment point with respect to centre of mass, $\mathrm{m}$ \\
\hline$\theta$ & Angle, deg \\
\hline$\omega$ & Angular rate, $\mathrm{deg} / \mathrm{s}$ \\
\hline $\mathbf{q}$ & Quaternion \\
\hline $\mathbf{r}$ & Position vector components, $\mathrm{m}$ \\
\hline $\mathbf{v}$ & Velocity vector components, m/s \\
\hline $\mathbf{a}$ & Acceleration vector components, $\mathrm{m} / \mathrm{s}^{2}$ \\
\hline$k$ & Stiffness, N/m \\
\hline$c$ & Damping coefficient, $\mathrm{N} \cdot \mathrm{s} / \mathrm{m}$ \\
\hline $\mathbf{K}_{P}$ & Proportional gain matrix \\
\hline $\mathbf{K}_{D}$ & Derivative gain matrix \\
\hline $\mathbf{I}_{x \times x}$ & Identity matrix of dimension $x$ \\
\hline$\phi$ & Tether angle, deg \\
\hline $\mathbf{H}$ & Thrust distribution matrix \\
\hline e & Error signal \\
\hline
\end{tabular}




$\begin{array}{ll}\hat{u} & \text { Thruster duty cycle } \\ \dot{x} & \text { Time derivative of } x \\ \ddot{x} & \text { Second time derivative of } x \\ \vec{x} & \text { Physical vector } x \\ \|\mathbf{x}\| & \text { Euclidean norm of } \mathbf{x} \\ \mathbf{x} & \text { Three dimensional components of physical vector } \vec{x}\end{array}$

$\begin{array}{ll}\text { Subscript } & \\ I & \text { Inertial reference frame } \\ t, j, c & \text { Body-fixed reference frame } \\ i & \text { Refers to target, junction, and chaser, respectively } \\ s 1 & i^{\text {th }} \text { sub-tether } \\ s 2 & \text { Due to first sub-tether } \\ m & \text { Due to second sub-tether } \\ s & \text { Due to the main tether } \\ g & \text { Due to the single tether } \\ x & \text { Due to gravity } \\ y & \text { Component in the } \vec{I}_{x} \text { direction } \\ \text { single } & \text { Component in the } \vec{I}_{y} \text { direction } \\ \text { sub } & \text { Referring to the single tether }\end{array}$

Superscript

$\mathrm{T}$

t

$c \quad$ Chaser body-fixed frame

$b \quad$ Body-fixed frame

$\times \quad$ Skew-symmetric matrix
Transpose
Target body-fixed frame 


\section{Chapter 1}

\section{Introduction}

\subsection{Motivation}

In early 2015, there were more than 17,000 objects larger than $10 \mathrm{~cm}$ in orbit around the Earth, but only $23 \%$ of them were operational spacecraft [1]. The defunct spacecraft that remain in orbit are named space debris. Due to their extremely high speeds, a collision between space debris, or between a space debris and a functional satellite, will cause both objects to break into many more debris. Leaving non-operational spacecraft in orbit was not considered a problem until 1978 when Kessler studied the collision frequency of spacecraft and found that while the density of debris in low Earth orbit is small, the collision frequency is non-zero and the debris population indeed has exponential growth [2]. This phenomenon was later called the Kessler Syndrome, which describes how collisions between spacecraft significantly increase the number of space debris, further increasing the chance of a collision. More recent studies by the European Space Agency have revealed that 5 to 10 properly selected pieces of space debris must be actively removed per year for the current number of space debris to remain stable [3]. Active removal of debris requires an active chaser spacecraft to rendezvous and remove the problematic debris. The technology to ac-

tively capture and remove a debris is currently being researched and developed, and is the motivation behind this work.

\subsection{Problem Statement}

One of the most significant technical challenges related to capturing and removing space debris arises when a chaser spacecraft attempts to capture a tumbling space debris. If a space debris were spinning about a single axis, the chaser spacecraft could simply match the angular rate of the target such that there is no relative motion 
between the chaser and the debris. Once matched, the chaser could safely dock with the debris and subsequently use its thrusters to detumble the system. However, when the target is tumbling with angular rates about all three axes, it is no longer trivial to match the angular rate of the target and perform a simple docking.

Instead, a more aggressive approach must be taken. Such an approach consists of the chaser quickly attaching to the target at one instant, and then immediately attempting to detumble the target using the chaser's actuators. The goal of the stabilization phase is to detumble the spinning target quickly, to allow for safe transportation of the debris to its disposal orbit or into the atmosphere.

This thesis is concerned with the stabilization phase of a space debris capture mission.

\subsection{Previous Work}

There has been significant previous work on the subject of space debris disposal. This section presents relevant previous work on the subject of space debris removal in order to position the contributions of this thesis in the context of the work that has already been done on the subject.

Reports have outlined various techniques and associated challenges for actively removing space debris $[1,3,4]$. Most techniques involve a chaser spacecraft that performs a rendezvous with the target debris. After rendezvous, either a rigid or flexible connection is made with the debris. This connection is used to stabilize the likely spinning debris [4], and then tow or push the debris into a lower orbit where it is released to decay into the atmosphere.

The research is highly specific to the technique used to capture the debris. Therefore, this literature review will be divided into sections depending on the capture technique used. Connecting a chaser to a target can be broken into two categories, namely rigid connections and flexible connections.

Rigid connections often use a mechanical arm [5], a combination of mechanical arms [6], or a grapple [7]. Difficulties with rigid connections occur when the debris has angular rates about all three axes. However, due to the major axis rule [8], which states that the minimum energy state of a rotating body is a spin solely about its 
major axis of inertia, combined with continual energy dissipation due to the flexible nature of all real structures, eventually all debris will have a single axis spin about their major axis of inertia [4]. For this reason, a significant amount of research is being performed on using robotic manipulators to capture debris [5-7,9]. However, in the short term it is possible for debris to be tumbling about all three axes. Therefore, in order to capture a general piece of debris, one must be able to capture a tumbling object. Along with rigid connections between the chaser and target, flexible connections are also possible.

There are three main techniques for deorbiting debris using flexible connections, namely: harpoons, electrodynamic tethers, or a net and tether system. Excellent overviews of flexible connections can be found in [10-12]. First, a harpoon can be used to establish a tethered connection between the chaser and the debris. This method involves launching a harpoon from the chaser that penetrates the insulation of the target and becomes embedded in the target $[3,13]$. A flexible tether attaches the harpoon to the chaser. Once the harpoon is embedded in the target, the chaser can then use its thrusters to tow the target to its disposal orbit. An on-orbit harpoon demonstration mission is planned for 2017 [14].

Electrodynamic tethers are another approach and have the benefit of being purely passive once initiated. A chaser attaches a multi-kilometer conductive tether to the target debris, that hangs in the nadir direction. The electrodynamic tether captures and conducts ions through its length. This current produces an electromagnetic field around the tether that interacts with the Earth's magnetic field, producing a deorbiting thrust [15-18]. The chaser is not connected to the electrodynamic tether, so it is free to pursue other debris once it attaches the tether to the target.

The third, and perhaps most universal capture technique, is to use a net and tether system. A net is launched from a chaser spacecraft and envelops a target. A tether is attached to both the net and the chaser such that the chaser can use its thrusters to have some control over the target. Advantages to the net and tether technique are that it does not require a specific attachment point on the target, it can capture various sizes of targets, the targets can have angular rates about all three axes, and an extremely close approach between the chaser and the target is not required. 
Net capturing research is highly active at present. Simulations [12,19-23] and experiments $[14,24-26]$ have been performed to understand how a net wraps around a target and how it remains secured. This thesis does not consider how the Tethered Spacecraft System (TSS) was established, but assumes that a tethered connection between the chaser and target was created either using a net or harpoon. All the following references have used this assumption as well. Aslanov and Yudintsev have investigated the dynamics of the TSS [27,28], the contribution of flexible appendages [29,30], and the contribution of fuel sloshing on the target [31]. Aslanov and Ledkov [32] studied atmospheric effects, and Aslanov [33] investigated the chaotic behaviour of the TSS. Recently, Yudintsev and Aslanov [34] proposed a target detumbling approach using tethered, liquid filled yo-yo masses. On-line techniques to estimate of the mass properties of a target debris when tethered have also been proposed [35], and adaptive backstepping control to detumble a debris is presented in [36] (with the assumption of an inelasic tether). Experiments have been performed that show the ability to perform formation flying for clusters of tethered spacecraft [37], while other experiments have tested debris towing strategies using rudimentary tethered hovercraft [38]. The experiments most relevant to this thesis have shown the resulting motion of two tethered spacecraft under static initial conditions in an air-bearing facility. One experiment towed a pre-stabilized debris, and another experiment towed a pre-stabilized debris with an initial offset in its attitude. Detumbling a spinning debris was not attempted. Also, significant discrepancies between the experimental and simulated results were reported [39]. Experiments concerning the stabilization of a spinning debris using flexible tethers have not been performed. The assumption of the tethers being rigidly attached to the target is also widely used to investigate the control aspects of towing a debris out of orbit using a TSS [38,40-44]. The towing phase of the mission begins after the target has been both securely captured and stabilized. The transient portion of the debris capture mission between when the net or harpoon attaches to the target and when the towing phase begins, i.e., the target detumbling phase, is often neglected.

As outlined above, throughout the considerable past research on active debris removal, it is often assumed that the debris is initially not spinning. The research 




Figure 1.1: Currently-accepted tether configuration ${ }^{1}$.

addressing the detumbling of debris has been complex and entirely theoretical thus far.

\subsection{Thesis Objectives}

This work examines the target detumbling phase of a space debris removal mission. Specifically, this work investigates the stabilization of a tumbling target with flexible tethers immediately after the target has been captured. In contrast to previous works that are complex and theoretical, this work seeks to drastically simplify the debris detumbling phase of the debris capture mission by altering the tether configuration used.

To accomplish this, a novel tether configuration is presented and compared to the presently accepted tether configuration. The currently accepted tether configuration consists of a single flexible tether joining a chaser spacecraft to an uncooperative debris, as shown in Fig. 1.1. The novel tether configuration consists of a single tether that attaches to the chaser but is split into smaller segments that are attached to the target at multiple locations. This configuration better exploits the damping properties of the tether which increases the rate at which angular momentum can be dissipated from the debris.

On-orbit numerical simulations are presented to demonstrate the improved detumbling ability of the proposed tether configuration. Experimental validation of the work is desired, so a new laboratory facility at Carleton University was developed for this purpose. The dynamics of the TSS in the laboratory environment are simulated, and are then validated through experiments.

\footnotetext{
${ }^{1}$ ESA, Space debris story, 2013 (accessed February 23, 2017) http://www.esa.int/ spaceinvideos/Videos/2013/04/Space_debris_story
} 


\subsection{Contributions}

The contributions of this work to the field of tethered space debris removal are:

- A novel tether configuration that simplifies the target detumbling phase of the debris capture mission.

- A new technique for detumbling debris is demonstrated. That is, spinning the TSS such that the centripetal force maintains tension in the tether necessary to detumble the debris.

- The development of a new laboratory facility that allows for experimentation of debris capture technologies.

- Using the above facility, the first experimental detumbling of a debris using tethers.

- Incorporation of a nonlinear tether stiffness model in simulation. 


\subsection{Organization}

This thesis is organized as follows:

To compare a novel tether configuration to the presently-accepted configuration, Chapter 2: On-Orbit Detumbling presents the equations of motion and tether models for a chaser tethered to a debris in Low Earth Orbit. It then presents seven numerical simulations that were performed comparing the two tether configurations, and comments on the results.

The development of an experimental facility, the Spacecraft Proximity Operations Testbed, is presented in Chapter 3: Experimental Facility, and was developed to allow for planar experimental validation of space dynamics, guidance, navigation, and control theories. In the context of this research, the facility was used to test the detumbling of space debris. This chapter provides a high-level overview of all the systems present, the author's contribution to these systems, and how they were set up in order to perform the space debris detumbling experimental validation. Full details of the laboratory work performed is available in Appendix A, and is intended as a reference for future students.

Chapter 4: Planar Detumbling presents the planar equations of motion for a tethered spacecraft system, similar to the environment present in the laboratory facility. Numerical simulations are presented that compare the two tether configurations, and the results are discussed.

Experimental methodologies and results are shown in Chapter 5: Experimental Validation. The scenarios that were numerically simulated in Chapter 4 are reproduced in experiments. Results are discussed and compared to the simulated results.

Chapter 6: Conclusion ends this thesis with a conclusion, summary of the contributions of this work, a list of publications that have resulted from this research, a comment on the practicality of the proposed debris removal strategy, and suggestions on future work on the topic. 


\section{Chapter 2}

\section{On-Orbit Detumbling}

This chapter presents the dynamics modeling of a tethered spacecraft system (TSS) in Low Earth Orbit, which includes gravity-gradient torques and orbital motion, for both a single tether and the proposed sub-tether configuration. Following this, numerical simulations are presented to demonstrate possible detumbling techniques and the benefits of the sub-tether configuration.

\section{$2.1 \quad$ Introduction}

High-fidelity numerical simulations are useful for understanding how a tethered spacecraft system behaves immediately after a tumbling debris is captured. Therefore, it is desirable to accurately model the dynamics of spacecraft in orbit and any perturbations that may be present on the spacecraft such that accurate capture scenarios can be investigated. This section presents the dynamics of two tethered spacecraft in orbit. Effects included, described in the following subsections, are: tether forces, gravity-gradient torques, and two-body orbital motion.

\subsection{On-orbit Dynamics}

The currently-accepted tethered spacecraft system consists of an active satellite (chaser) connected to a debris (target) via a visco-elastic tether. Figure 2.1(a) shows a typical, previously-studied $[12,14,19-44]$, configuration. A tether connects the chaser to an attachment point on the target. The proposed configuration is shown in Fig. 2.1(b), and has a tether attached to the chaser which branches into four smaller tethers that are attached to the target at various locations. The tether leaving the chaser will be denoted as the main tether and the four tethers connecting the main tether to the 


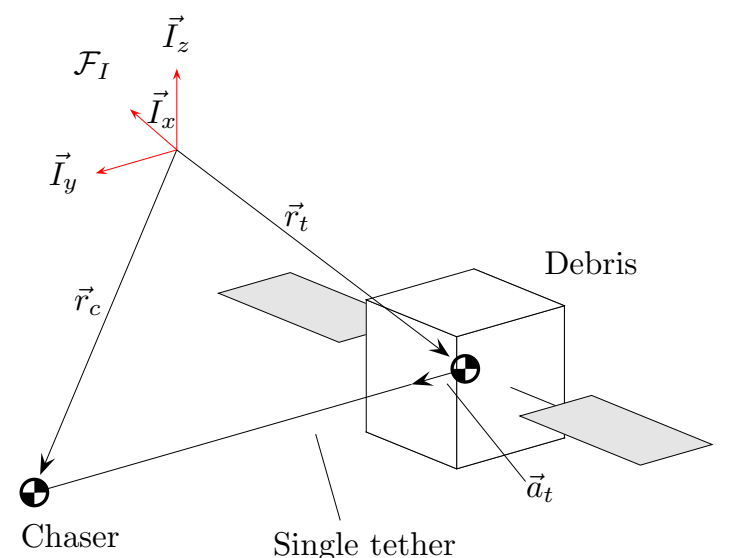

Chaser
Single tether



(a) Single tether configuration

(b) Sub-tether configuration

Figure 2.1: Reference frame and vector definition for dynamics modeling.

target are denoted as sub-tethers. The point where the main tether splits into subtethers is denoted the junction. The tethers are modeled as massless spring-dampers with torsion and bending effects ignored. The single tether is assumed to be perfectly attached to the debris at the centre of the front panel, as shown in Fig. 2.1(a), and the sub-tethers are assumed to be perfectly attached to the extremities of the front panel on the debris, as shown in Fig. 2.1(b). The approach taken herein is to use the spring-damper properties of the main tether and sub-tethers to regulate the angular momentum of an uncontrolled target, in a Low Earth Orbit (LEO) environment. The purpose of this chapter is to demonstrate how the proposed novel TSS provides an improvement in the stabilizing ability compared to the currently-accepted TSS configuration.

\subsubsection{Dynamics Formulation - Sub-Tether Configuration}

Referring to Fig. 2.1(b), the reference frame $\mathcal{F}_{I}$ represents an inertially-fixed reference frame. The position of the target is described by position vector $\vec{r}_{t}$, such that:

$$
\vec{r}_{t}=\overrightarrow{\mathcal{F}}_{I}^{\mathrm{T}} \mathbf{r}_{t}
$$


where, $\mathbf{r}_{t}$ represents the three-dimensional components of the target centre of mass position vector in the inertially fixed reference frame $\mathcal{F}_{I}$. The junction and chaser are represented as point masses, their locations described by position vectors $\vec{r}_{c}$ and $\vec{r}_{j}$, respectively, such that:

$$
\begin{aligned}
& \vec{r}_{c}=\overrightarrow{\mathcal{F}}_{I}^{\mathrm{T}} \mathbf{r}_{c} \\
& \vec{r}_{j}=\overrightarrow{\mathcal{F}}_{I}^{\mathrm{T}} \mathbf{r}_{j}
\end{aligned}
$$

where $\mathbf{r}_{c}$ and $\mathbf{r}_{j}$ are the three-dimensional components of the chaser and junction position vectors in the inertially fixed reference frame $\mathcal{F}_{I}$, respectively.

\subsubsection{Attitude Motion}

Euler's equations of motion are used to describe the target angular rates in the target body-fixed reference frame $\mathcal{F}_{B}$, given by [8]

$$
\mathbf{J} \dot{\omega}+\omega^{\times}(\mathbf{J} \boldsymbol{\omega})=\tau
$$

where $\boldsymbol{\omega}$ are the components of the angular rate vector in $\mathcal{F}_{B}$, and $\boldsymbol{\tau}$ is the external torque applied to the target.

Assuming that the target body-fixed frame, $\mathcal{F}_{B}$, is aligned with the principal axes of the body, the target inertia matrix, denoted as $\mathbf{J}$, is represented by:

$$
\mathbf{J}=\left[\begin{array}{ccc}
J_{x x} & 0 & 0 \\
0 & J_{y y} & 0 \\
0 & 0 & J_{z z}
\end{array}\right]
$$

where $J_{x x}, J_{y y}$, and $J_{z z}$ are the principal moments of inertia of the target debris.

The attitude of the target is described by quaternion kinematics, given by:

$$
\dot{\mathbf{q}}=\frac{1}{2}\left[\begin{array}{c}
\left(\boldsymbol{\epsilon}^{\times}+\eta \mathbf{I}_{3 \times 3}\right) \boldsymbol{\omega} \\
-\boldsymbol{\epsilon}^{\mathrm{T}} \boldsymbol{\omega}
\end{array}\right]
$$

where, $\mathbf{q}$ represents the quaternion describing the attitude of $\mathcal{F}_{B}$ with respect to $\mathcal{F}_{I}$ and is defined as: 


$$
\mathbf{q}=\left[\begin{array}{c}
q_{1} \\
q_{2} \\
q_{3} \\
q_{4}
\end{array}\right]=\left[\begin{array}{l}
\boldsymbol{\epsilon} \\
\eta
\end{array}\right]=\left[\begin{array}{c}
\mathbf{b} \cos \left(\frac{\psi}{2}\right) \\
\sin \left(\frac{\psi}{2}\right)
\end{array}\right]
$$

where

$$
\boldsymbol{\epsilon}=\left[\begin{array}{l}
q_{1} \\
q_{2} \\
q_{3}
\end{array}\right]
$$

where $\mathbf{b}$ is the axis of rotation and $\phi$ is the angle of rotation [8].

The inverse square law of the gravitational field of the Earth creates perturbation torques on a body, that are significant in LEO. The unequal gravitational force acting across the body leads to restoring torques on the spacecraft [8]. The effect is quantified through:

$$
\boldsymbol{\tau}_{g}=\frac{3 \mu}{\left\|\mathbf{r}_{t}^{b}\right\|^{5}} \mathbf{r}_{t}^{b \times} \mathbf{J r}_{t}^{b}
$$

where $\boldsymbol{\tau}_{g}$ is the resulting gravity-gradient torque components in $\mathcal{F}_{B}, \mu$ is the gravitational parameter of Earth, and $\mathbf{r}_{t}^{b}$ are the components of the target position vector expressed in $\mathcal{F}_{B}$, via

$$
\mathbf{r}_{t}^{b}=\mathbf{A}(\mathbf{q}) \mathbf{r}_{t}
$$

The rotation matrix $\mathbf{A}(\mathbf{q})$ represents a rotation from $\mathcal{F}_{I}$ to $\mathcal{F}_{B}$, which is obtained from the quaternion through:

$$
\mathbf{A}(\mathbf{q})=\left[\begin{array}{ccc}
1-2 q_{2}^{2}-2 q_{3}^{2} & 2\left(q_{1} q_{2}+q_{4} q_{3}\right) & 2\left(q_{1} q_{3}-q_{4} q_{2}\right) \\
2\left(q_{1} q_{2}-q_{4} q_{3}\right) & 1-2 q_{1}^{2}-2 q_{3}^{2} & 2\left(q_{2} q_{3}+q_{4} q_{1}\right) \\
2\left(q_{1} q_{3}+q_{4} q_{2}\right) & 2\left(q_{2} q_{3}-q_{4} q_{1}\right) & 1-2 q_{1}^{2}-2 q_{2}^{2}
\end{array}\right]
$$

\subsubsection{Translational Motion}

To model the target, junction, and chaser linear motion, Newton's second law is used:

$$
\mathbf{F}=m \mathbf{a}
$$


where $\mathbf{F}$ is the net inertial external force applied to the body, $m$ is the mass of the body, and $\mathbf{a}$ is the inertial linear acceleration.

In the TSS shown in Fig. 2.1(b), the main tether and sub-tethers are generating external forces that are applied to each mass. The tether is modeled as a simple springdamper system, as shown in Fig. 2.2. Defining the $i^{\text {th }}$ sub-tether under analysis by

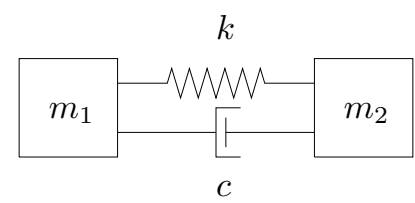

Figure 2.2: Simple spring-damper system, where two masses are attached via a spring and damper in parallel.

the vector $\vec{L}_{i}$, in $\mathcal{F}_{I}$ has components of:

$$
\mathbf{L}_{i}=\mathbf{r}_{j}-\mathbf{r}_{t}-\mathbf{A}(\mathbf{q})^{\mathrm{T}} \mathbf{a}_{i}, \quad \forall i=1, \ldots, 4
$$

where $\mathbf{a}_{i}$ is the attachment point of the $i^{t h}$ sub-tether to the target, with respect the the centre of the target, in $\mathcal{F}_{B}$. Treating the linear spring and damper in parallel, the resultant tensile force magnitude developed by the $i^{\text {th }}$ sub-tether is:

$$
F_{i}= \begin{cases}k\left(\left\|\mathbf{L}_{i}\right\|-L_{0}\right)+c\left[\mathbf{v}_{j}-\left(\mathbf{v}_{t}+\mathbf{A}(\mathbf{q})^{\mathrm{T}} \boldsymbol{\omega}^{\times} \mathbf{a}_{i}\right)\right]^{\mathrm{T}} \frac{\mathbf{L}_{i}}{\left\|\mathbf{L}_{i}\right\|}, & \text { for }\left\|\mathbf{L}_{i}\right\|-L_{0}>0 \\ 0, & \text { otherwise }\end{cases}
$$

where $\mathbf{v}_{j}$ and $\mathbf{v}_{t}$ are velocity components of the junction and target in $\mathcal{F}_{I}$, respectively. The tether material is assumed to have a linear spring constant, $k$, damping coefficient, $c$, and unstretched length $L_{0}$.

The gravitational force on an orbiting satellite is described by Newton's law of gravitation:

$$
\mathbf{F}_{g}=-\frac{\mu m}{\|\mathbf{r}\|^{3}} \mathbf{r}
$$

where $\mu$ is the gravitational parameter of the Earth, $m$ is the mass of the orbiting satellite, $\mathbf{r}$ is the components of the position vector of the orbiting satellite in $\mathcal{F}_{I}$, and 
$\mathbf{F}_{g}$ is the resulting force vector components. Newton's law of gravitation applies to the chaser, junction, and target components of the TSS.

\subsubsection{Equations of Motion}

To properly model the motion, all forces contributing to translational motion are included in Eq. (2.11) for each body. The chaser and junction are treated as point masses, so the effects of external torques are modeled using Eq. (2.4) for the target only.

The resulting net force on the target debris in $\mathcal{F}_{I}$ is due to the four sub-tethers and the gravitational effects, and is given by:

$$
\mathbf{F}_{t}=\mathbf{F}_{s}+\mathbf{F}_{g}=\sum_{i=1}^{4} F_{i} \frac{\mathbf{L}_{i}}{\left\|\mathbf{L}_{i}\right\|}-\frac{\mu m_{t}}{\left\|\mathbf{r}_{t}\right\|^{3}} \mathbf{r}_{t}
$$

Similarly, for the chaser spacecraft, with the addition of its own thrust force, $\mathbf{F}_{\text {thrust }}$

$$
\mathbf{F}_{c}=-\mathbf{F}_{m}+\mathbf{F}_{g}+\mathbf{F}_{\text {thrust }}=-F_{m} \frac{\mathbf{L}_{m}}{\left\|\mathbf{L}_{m}\right\|}-\frac{\mu m_{c}}{\left\|\mathbf{r}_{c}\right\|^{3}} \mathbf{r}_{c}+\mathbf{F}_{\text {thrust }}
$$

where, $F_{m}$ denotes the force magnitude present in the main tether and $\mathbf{L}_{m}$ denotes its vector components. The junction experiences similar forces:

$$
\mathbf{F}_{j}=\mathbf{F}_{m}-\mathbf{F}_{s}+\mathbf{F}_{g}=F_{m} \frac{\mathbf{L}_{m}}{\left\|\mathbf{L}_{m}\right\|}-\sum_{i=1}^{4} F_{i} \frac{\mathbf{L}_{i}}{\left\|\mathbf{L}_{i}\right\|}-\frac{\mu m_{j}}{\left\|\mathbf{r}_{j}\right\|^{3}} \mathbf{r}_{j}
$$

The torque imparted on the target is the sum of the four sub-tether contributions and the gravity-gradient effects, given by:

$$
\boldsymbol{\tau}_{t}=\boldsymbol{\tau}_{s}+\boldsymbol{\tau}_{g}=\mathbf{A}(\mathbf{q}) \sum_{i=1}^{4} F_{i} \frac{\mathbf{a}_{i}^{\times} \mathbf{L}_{i}}{\left\|\mathbf{L}_{i}\right\|}+\frac{3 \mu}{\left\|\mathbf{r}_{t}^{b}\right\|^{5}} \mathbf{r}_{t}^{b \times} \mathbf{J r}_{t}^{b}
$$

which is defined in $\mathcal{F}_{B}$. To simulate the dynamics, Eqs. (2.15) to (2.18) are solved numerically with Eqs. (2.4) and (2.11). 


\subsubsection{Dynamics Formulation - Single Tether Configuration}

The dynamics equations for the single tether configuration are very similar to the sub-tether configuration. The major difference is the absence of the junction point, as shown in Fig. 2.1. Therefore, instead of Eq. (2.12), the tether vector components in $\mathcal{F}_{I}$ becomes

$$
\mathbf{L}=\mathbf{r}_{c}-\mathbf{r}_{t}-\mathbf{A}(\mathbf{q})^{\mathrm{T}} \mathbf{a}_{t}
$$

Here, $\mathbf{a}_{t}$ is the single tether contact point on the target with respect to the centre of the target, as shown in Fig. 2.1(a). The force in the tether is:

$$
F= \begin{cases}k\left(\|\mathbf{L}\|-L_{0}\right)+c\left[\mathbf{v}_{c}-\left(\mathbf{v}_{t}+\mathbf{A}(\mathbf{q})^{\mathrm{T}} \boldsymbol{\omega}^{\times} \mathbf{a}_{t}\right)\right]^{\mathrm{T}} \frac{\mathbf{L}}{\|\mathbf{L}\|}, & \text { for }\|\mathbf{L}\|-L_{0}>0 \\ 0, & \text { otherwise }\end{cases}
$$

The resulting forces and torques on each body are:

$$
\mathbf{F}_{t}=\mathbf{F}_{m}+\mathbf{F}_{g}=F \frac{\mathbf{L}}{\|\mathbf{L}\|}-\frac{\mu m_{t}}{\left\|\mathbf{r}_{t}\right\|^{3}} \mathbf{r}_{t}
$$

for the force on the target,

$$
\mathbf{F}_{c}=-\mathbf{F}_{m}+\mathbf{F}_{g}+\mathbf{F}_{\text {thrust }}=-F \frac{\mathbf{L}}{\|\mathbf{L}\|}-\frac{\mu m_{c}}{\left\|\mathbf{r}_{c}\right\|^{3}} \mathbf{r}_{c}+\mathbf{F}_{\text {thrust }}
$$

for the force on the chaser, and

$$
\boldsymbol{\tau}_{t}=\boldsymbol{\tau}_{m}+\boldsymbol{\tau}_{g}=\mathbf{A}(\mathbf{q}) F \frac{\mathbf{a}_{t}^{\times} \mathbf{L}}{\|\mathbf{L}\|}+\frac{3 \mu}{\left\|\mathbf{r}_{t}^{\mathrm{b}}\right\|^{5}} \mathbf{r}_{t}^{b \times} \mathbf{J r}_{t}^{b}
$$

for the torque on the target. The forces and torques in Eqs. (2.21) to (2.23) are solved numerically with Eqs. (2.4) and (2.11) in simulation.

\subsection{On-Orbit Numerical Simulations}

This section presents numerical simulations for a tethered spacecraft system in LEO immediately after a tumbling space debris has been captured. Seven simulations are presented with varying initial conditions and stabilization techniques. Those are: 
1. Chaser continually thrusts away from the target. Target has a single axis spin aligned with the chaser. Tether angle is initially non-zero. Tethers are initially tight. Results are presented in Section 2.3.1.

2. Identical to Case 1 except the tethers are initially slack. Results are presented in Section 2.3.2.

3. Chaser continually thrusts away from the target with a low thrust engine. Target has a single axis spin aligned with the chaser. The tether angle is initially zero, and the tethers are initially tight. Results are presented in Section 2.3.3.

4. Chaser continually thrusts away from the target. Target is initially tumbling. Tether angle is initially zero. Tethers are initially tight. Results are presented in Section 2.3.4.

5. Chaser continually thrusts away from the target. Target is initially tumbling. Tether angle is initially non-zero. Tethers are initially slack. This constitutes the most extreme capture scenario simulated. Results are presented in Section 2.3.5.

6. This case uses a different technique to stabilize the tumbling target. Instead of continually thrusting away from the debris, a spin of the TSS is initiated such that the centripetal force maintains tension in the tethers and stabilizes the target. Here, the target is initially tumbling, the tether angle is initially zero, and the tethers are initially tight. Results are presented in Section 2.3.6.

7. The final simulation investigates the natural tendencies of the TSS. Here, the target has no angular rates (as if it were already stabilized). The tethers are initially tight, and the tether angle is zero. The chaser does not use its thrusters. This case examines whether a stabilized system remains stable in orbit. Results are presented in Section 2.3.7.

Each case compares the target stabilization ability of the single tether configuration to the sub-tether configuration. The orbital elements selected for all simulations are listed in Table 2.1. The orbital elements are converted into the position and velocity 
Table 2.1: Orbital elements used to define initial orbit of TSS.

\begin{tabular}{cc}
\hline \hline Orbital Element & Value \\
\hline Semi-major axis, $a$ & $6871 \mathrm{~km}$ \\
Eccentricity, $e$ & 0.001 \\
Right ascension of & $20 \mathrm{deg}$ \\
the ascending node, $\Omega$ & \\
Inclination, $i$ & $60 \mathrm{deg}$ \\
Argument of perigee, $\omega$ & $90 \mathrm{deg}$ \\
True anomaly, $\theta$ & $60 \mathrm{deg}$ \\
\hline \hline
\end{tabular}

initial conditions for use in simulation. To accomplish this, first the magnitude of the radius vector, $R$, is calculated as:

$$
R=\frac{a\left(1-e^{2}\right)}{1+e \cos \theta}
$$

the position and velocity vector components, $\mathbf{R}_{p}$ and $\mathbf{V}_{p}$ respectively, in the perifocal reference frame are then calculated as:

$$
\begin{gathered}
\mathbf{R}_{p}=\left[\begin{array}{c}
R \cos \theta \\
R \sin \theta \\
0
\end{array}\right] \\
\mathbf{V}_{p}=\left[\begin{array}{c}
-\sqrt{\frac{\mu}{a\left(1-e^{2}\right)}} \sin \theta \\
\sqrt{\frac{\mu}{a\left(1-e^{2}\right)}}(e+\cos \theta) \\
0
\end{array}\right]
\end{gathered}
$$

where $\mu$ is the gravitational parameter of the Earth. The $\mathbf{R}_{p}$ and $\mathbf{V}_{p}$ vectors are then rotated into $\mathcal{F}_{I}$ (where the simulation is performed) through the rotation matrix

$\mathbf{A}_{I P}=\left[\begin{array}{ccc}\cos \Omega \cos \omega-\sin \Omega \cos i \sin \omega & -\cos \Omega \sin \omega-\sin \Omega \cos i \cos \omega & \sin \Omega \sin i \\ \sin \Omega \cos \omega+\cos \Omega \cos i \sin \omega & -\sin \Omega \sin \omega+\cos \Omega \cos i \cos \omega & -\cos \Omega \sin i \\ \sin i \sin \omega & \sin i \cos \omega & \cos i\end{array}\right]$ 
and is used to obtain the initial conditions of:

$$
\begin{gathered}
\mathbf{R}_{I}=\mathbf{A}_{I P} \mathbf{R}_{P}=\left[\begin{array}{c}
-6176 \\
-421 \\
2974
\end{array}\right] \mathrm{km} \\
\mathbf{V}_{I}=\mathbf{A}_{I P} \mathbf{V}_{P}=\left[\begin{array}{c}
-2.46 \\
-4.40 \\
-5.71
\end{array}\right] \mathrm{km} / \mathrm{s}
\end{gathered}
$$

that are used as the initial conditions in the simulations.

Each simulation has similar properties and initial conditions, shown in Table 2.2. The differences between simulation cases are presented in the following subsections. The chaser is in an identical orbit as the target, but is behind the target by 30 meters, except for Cases 2 and 5, where is it behind by 27 meters to create a slack tether. This separation distance is simulated by imparting a small difference in true anomaly for the chaser spacecraft.

Table 2.2: Initial conditions and TSS parameters for simulations.

\begin{tabular}{cccccc}
\hline \hline Parameter & Value & Parameter & Value & Parameter & Value \\
\hline$J_{x x}, \mathrm{~kg} \cdot \mathrm{m}^{2}$ & 15000 & $m_{t}, \mathrm{~kg}$ & 3000 & Debris Size, $\mathrm{m}$ & {$[1.25,1.75,1.25]$} \\
$J_{y y}, \mathrm{~kg} \cdot \mathrm{m}^{2}$ & 3000 & $m_{j}, \mathrm{~kg}$ & 10 & $L_{\text {single }}, \mathrm{m}$ & 30 \\
$J_{z z}, \mathrm{~kg} \cdot \mathrm{m}^{2}$ & 15000 & $m_{c}, \mathrm{~kg}$ & 500 & $L_{\text {main }}, \mathrm{m}$ & 15.0 \\
$k, \frac{\mathrm{N}}{\mathrm{m}}$ & 3150 & $c, \frac{\mathrm{Ns}}{\mathrm{m}}$ & 16 & $L_{\text {sub }}, \mathrm{m}$ & 15.2 \\
\hline \hline
\end{tabular}

The simulations are performed using an Adams integration scheme over 6000 seconds (approximately one orbit), using a 1 second output time step. Output graphs show the resulting angular rates of the target as a function of time, to determine whether the target is effectively being controlled. The angle between the front-facenormal of the target and the single tether as a function of time, denoted $\phi$ as represented in Fig. 2.3, is also presented to show how close the attitude of the target is to its equilibrium point. The angle is calculated as follows:

$$
\phi=\cos ^{-1}\left(\frac{-\left(\mathbf{A}(\mathbf{q})^{\mathrm{T}} \mathbf{B}_{y}\right)^{\mathrm{T}} \mathbf{L}}{\left\|\mathbf{B}_{y}\right\|\|\mathbf{L}\|}\right)
$$




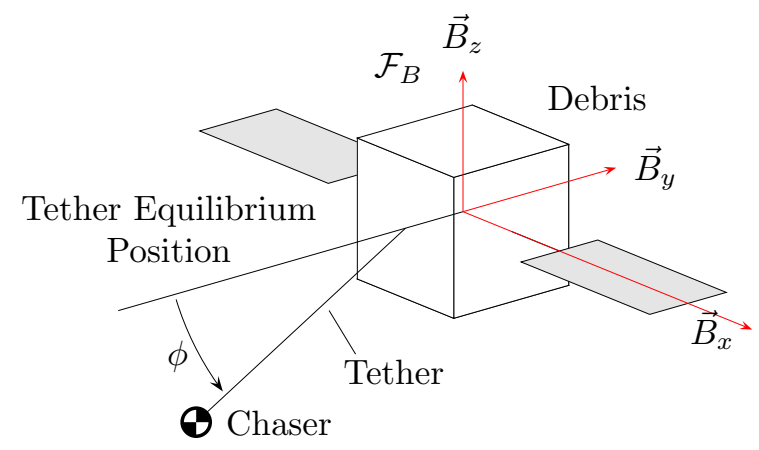

Figure 2.3: Angle $\phi$ represents the current angle between the tether and its equilibrium point. Reference frame $\mathcal{F}_{B}$ defines the body-fixed reference frame on the target debris.

where $\mathbf{B}_{y}$ is the components of the unit vector $\vec{B}_{y}$ expressed in $\mathcal{F}_{B}$, and $\mathbf{L}$ is the tether vector components in $\mathcal{F}_{I}$. The proposed sub-tether configuration uses a hypothetical single tether to calculate the angle, such that the two TSS solutions can be readily compared. The tether elongation as a function of time is presented to determine if tether oscillations are present.

\subsubsection{Case 1}

A debris is captured at $t=0$ when it has initial angular rates of:

$$
\boldsymbol{\omega}=\left[\begin{array}{c}
0 \\
0.05 \\
0
\end{array}\right] \frac{\mathrm{rad}}{\mathrm{s}}
$$

The debris is not captured perfectly, however. To simulate this, an initial tether angle of $\pi / 6$ radians is applied such that the target is not facing the chaser upon capture. The chaser thrusts $20 \mathrm{~N}$ in the retrograde direction, and is initially 30 meters behind the target in the orbit such that all tethers are initially tight.

This case shows that in the presence of a thrust force on the chaser, the angular rates of the target can be controlled. Figure 2.4(a) shows that sub-tether configuration damps the angular rates of the passive target faster than the single tether 


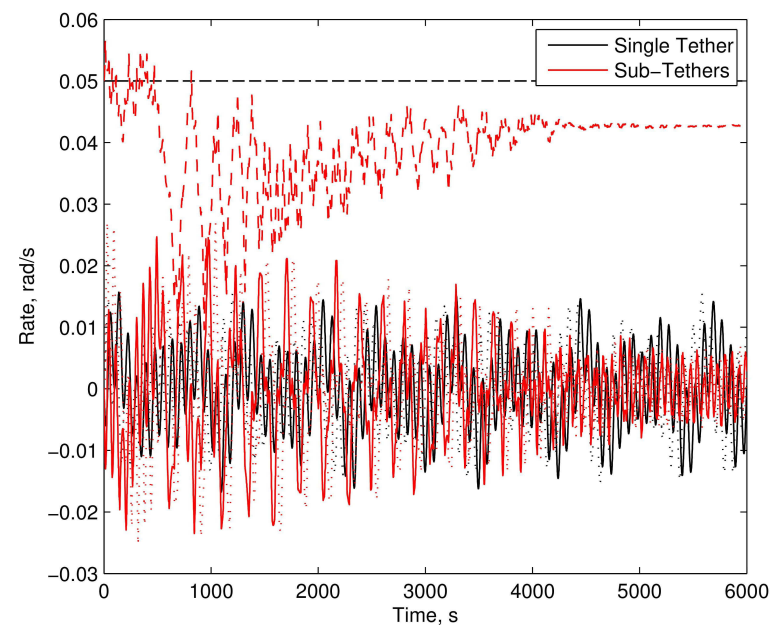

(a) Target angular rates

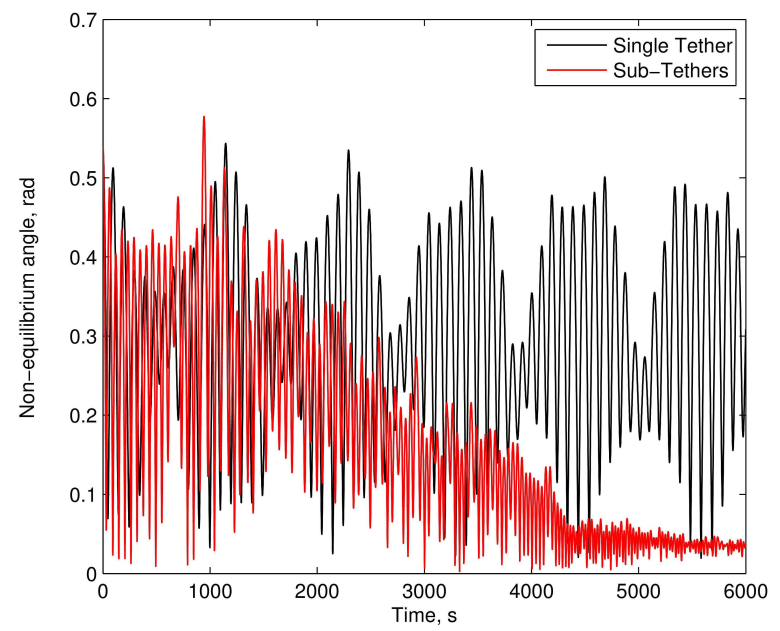

(b) Tether angle

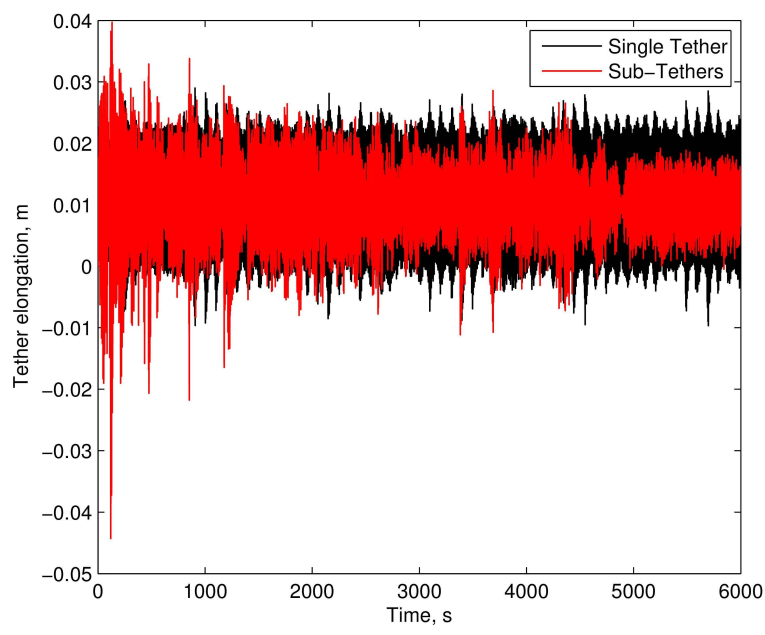

(c) Tether elongation

Figure 2.4: Resulting on-orbit TSS motion for Case 1. Dashed lines represent the angular rate about the $\vec{B}_{y}$ axis, and the dotted and solid lines represent the angular rate about the $\vec{B}_{x}$ and $\vec{B}_{z}$ axes, respectively, as shown in Fig. 2.3.

configuration. It is interesting to note that neither configuration can control the angular rates about the $\vec{B}_{y}$ axis, due to the lack of a moment arm about the $\vec{B}_{y}$ axis, as defined in Fig. 2.3. This is a limitation of the TSS debris removal solution, in that it cannot control the angular rates of the target about the axis joining the chaser and the target. The angular rates about the $\vec{B}_{x}$ and $\vec{B}_{z}$ axes approach zero, but they do not reach zero. The nature of the sub-tether configuration leads to a non-zero angular 
separation between when one sub-tether becomes tight and when an opposite subtether becomes tight. Therefore, small scale oscillations are expected to be present in steady state.

Another measure of the performance of the TSS is to show the angle between the tether and its equilibrium position, as depicted in Fig. 2.3. The sub-tether configuration uses a hypothetical single tether to calculate said angle, such that the target orientation can be compared. The angle is initially at $\pi / 6$ to simulate an imperfect capture process. Figure 2.4(b) shows that while the angle in the single tether case is decreasing over time, it does so very slowly compared to the sub-tether configuration angle. If the target is always facing the chaser (i.e., a small tether angle), there are multiple benefits. First, the target will have reduced oscillations. For a piece of debris that has been in orbit for an extended period of time, smaller oscillations may reduce the chance of break up. Second, the desired towing configuration is when the target is perfectly aligned with the chaser, so the quicker this configuration can be obtained the more efficient the TSS debris removal solution will be.

Figure 2.4(c) shows the tether elongation as a function of time. For the sub-tether scenario, the longest of the four sub-tethers (i.e., the one currently being stretched) is used in the calculation. Again, the sub-tether configuration outperforms the single tether scenario, with lower stretch amplitudes after the first few seconds. This shows that there will be lower oscillatory forces in the tether over time. The tether stretch tends towards a constant positive value which is a result of the constant thrust force provided by the chaser.

\subsubsection{Case 2}

A debris is captured at $t=0$, similar to Case 1. All initial conditions are identical except the chaser is initially 27 meters behind the target. The tethers are still 30 meters long, thereby creating an initial slackness in the tethers of 3 meters. As the chaser thrusts away from the target, there will be an initial jolt when the tethers becomes tight for the first time. Figure 2.5(a) shows how the angular rates become damped to their final values nearly as quickly as Case 1 . Therefore, Case 2 shows that capturing the target with an initially slack tether leads to a safe stabilization 
and that the sub-tether TSS configuration is still a better solution to the stabilization of a spinning target.

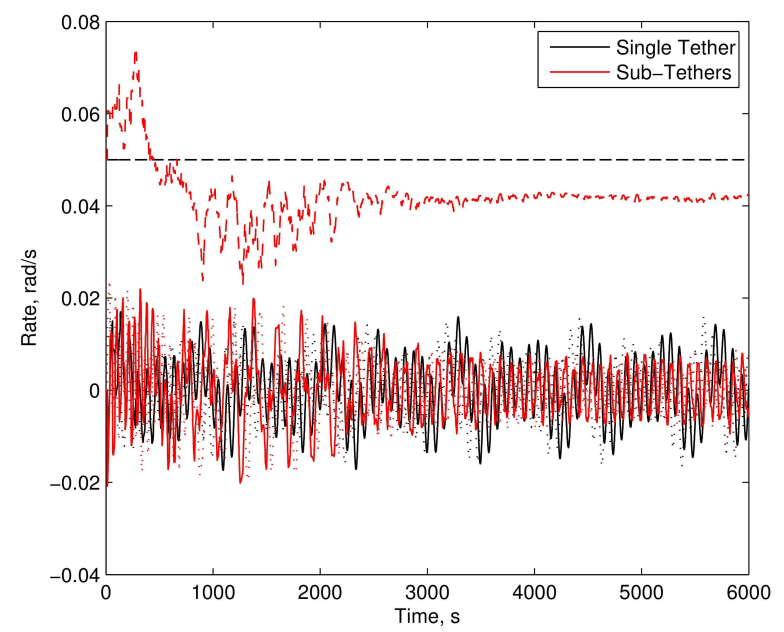

(a) Target angular rates

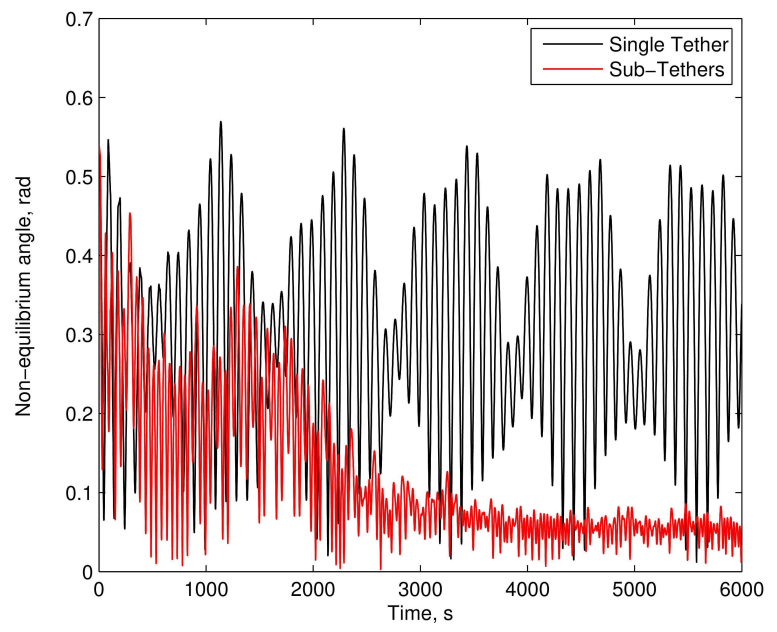

(b) Tether angle

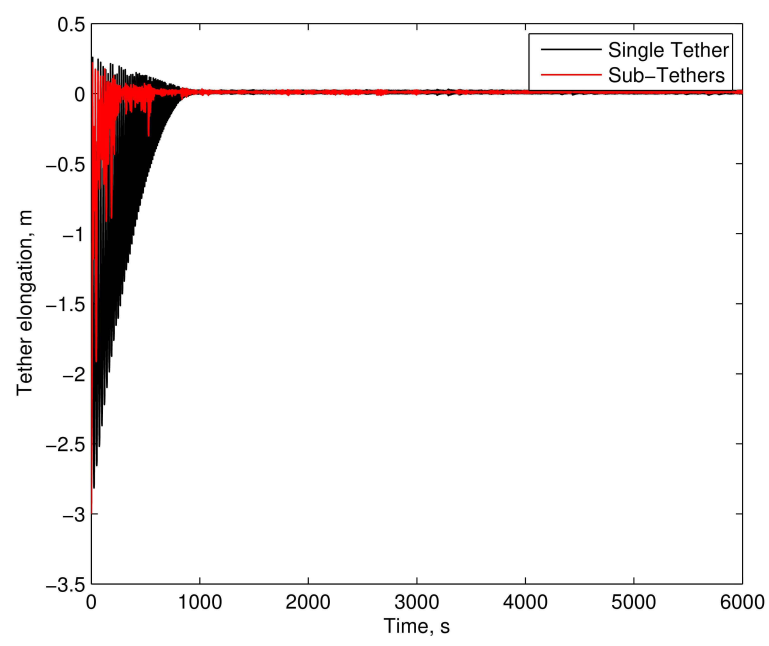

(c) Tether elongation

Figure 2.5: Resulting on-orbit TSS motion for Case 2

\subsubsection{Case 3}

Case 3 investigates the effects of a smaller $3 \mathrm{~N}$ thrust force from the chaser. This case considers a scenario where the chaser is towing the target with less thrust than was used during the stabilizing process. Here, the target is already facing the chaser, but it continues to have a naturally unstable spin about its minor (and uncontrollable) 
axis, of:



(a) Target angular rates

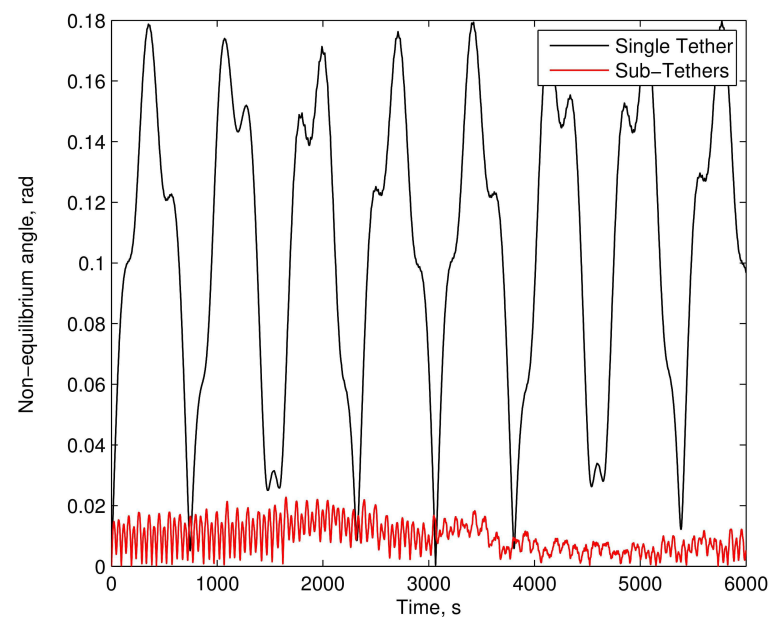

(b) Tether angle



(c) Tether elongation

Figure 2.6: Resulting on-orbit TSS motion for Case 3

$$
\boldsymbol{\omega}=\left[\begin{array}{c}
0 \\
0.05 \\
0
\end{array}\right] \frac{\mathrm{rad}}{\mathrm{s}}
$$

Figure 2.6(a) shows that as perturbations act on the target, its angular rates about the $\vec{B}_{x}$ and $\vec{B}_{z}$ axes remain small and bounded (i.e., stable) with either configuration. Figure 2.6(b) shows how the tether angle is considerably lower for the sub-tether configuration than the single tether configuration, which allows the target to remain 
nearer its equilibrium position during towing. The stretch for the sub-tether configuration, as shown in Fig. 2.6(c), is nearly constant for the sub-tether configuration. The single tether, however, develops oscillations due to the perturbations which result in slackness of the tether of up to 0.5 meters. Therefore, if the towing phase of the debris removal mission is planning on using a low-thrust engine, the sub-tether configuration is a better option as it keeps the target near its equilibrium position to ensure safe towing.

\subsubsection{Case 4}

Cases 4 - 6 consider the likely possibility of target angular rates about all three axes. Initial angular rates of:

$$
\boldsymbol{\omega}=\left[\begin{array}{l}
0.025 \\
0.025 \\
0.025
\end{array}\right] \frac{\mathrm{rad}}{\mathrm{s}}
$$

are used. This case has the target initially facing the chaser (i.e., zero tether angle), $20 \mathrm{~N}$ of thrust from the chaser, and 30 meters separation such that all tethers are initially tight. Figure 2.7 presents the results.

This case is more severe than Cases 1-3 because the target is tumbling with angular rates about all three axes. Figure 2.7(a) shows how after one orbit, the angular rates of the target using the sub-tether configuration are significantly reduced compared to the single tether configuration. This is due to the placement of the sub-tether attachment points on the target. By attaching the sub-tethers to the target at its extremities where the relative velocities are highest, as shown in Fig. 2.1(b), the damping effect in the sub-tethers is better utilized. Since damping is the only mode of energy dissipation in the system, attaching sub-tethers to the points of highest relative velocity is a more effective method of dissipating the angular momentum of a tumbling debris.

\subsubsection{Case 5}

This case has the same initial conditions as Case 4 except the target is initially offset from the chaser with an angle of $\pi / 6$ radians, and the tethers are initially 3 meters 


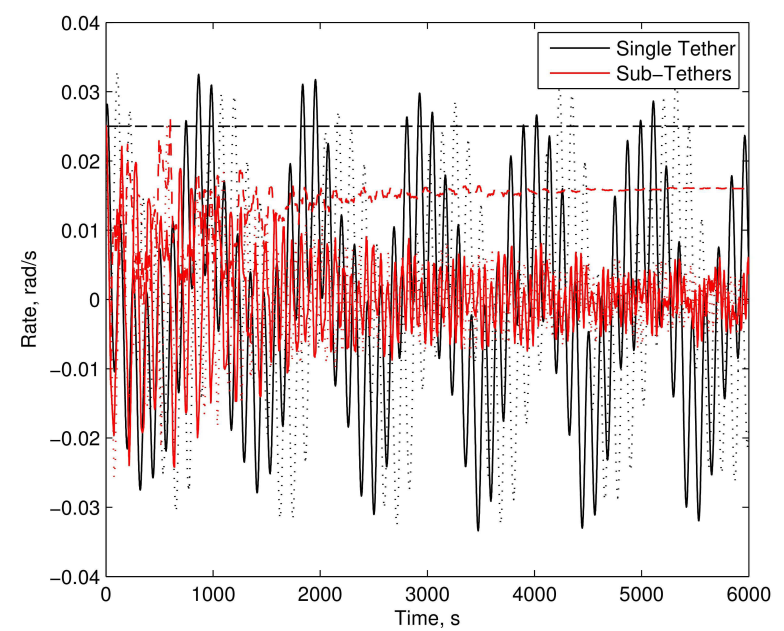

(a) Target angular rates

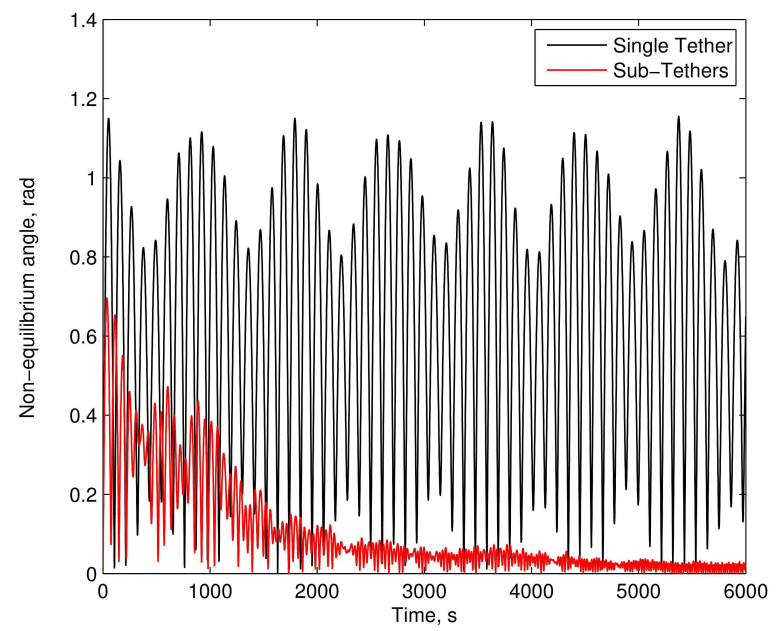

(b) Tether angle

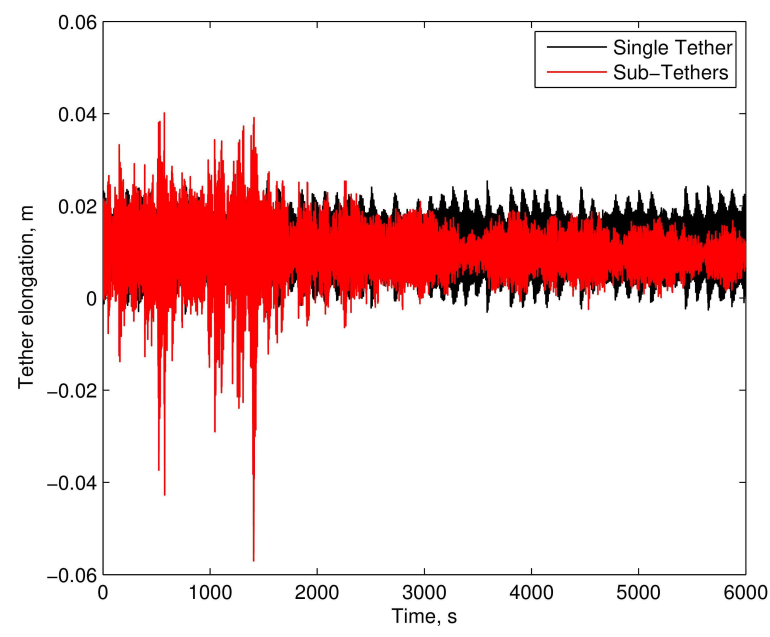

(c) Tether elongation

Figure 2.7: Resulting on-orbit TSS motion for Case 4

slack. As in Case 4, $20 \mathrm{~N}$ of thrust is used. This represents the most difficult capture scenario that was simulated. That is, an imperfect capture with an initial tether angle of $\pi / 6$ rads, an initially 3 meter slack tether, and a tumbling target with angular rates about all three axes. Figure 2.8 once again shows how the sub-tether configuration outperforms the single tether. Both the angular rates, the angular offset, and the tether elongation reach their steady state values much quicker than the single tether TSS, using the same amount of thrust. 


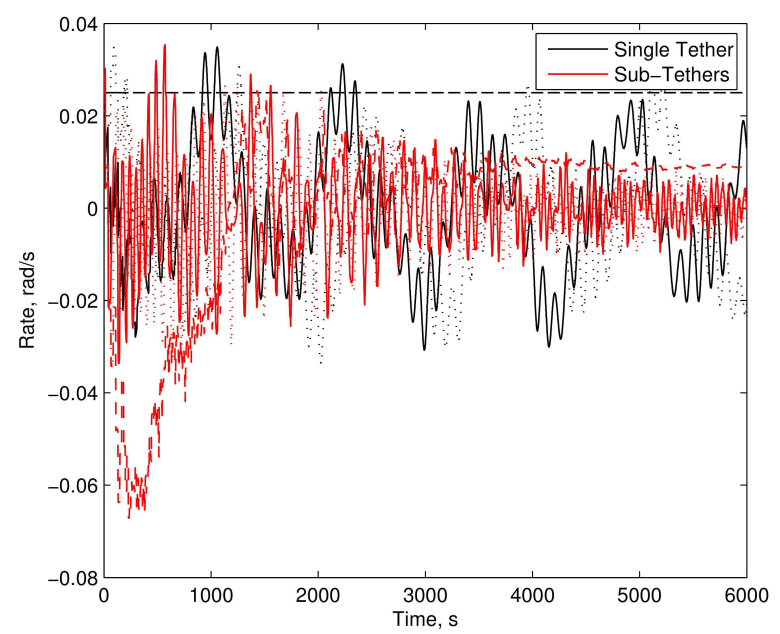

(a) Target angular rates

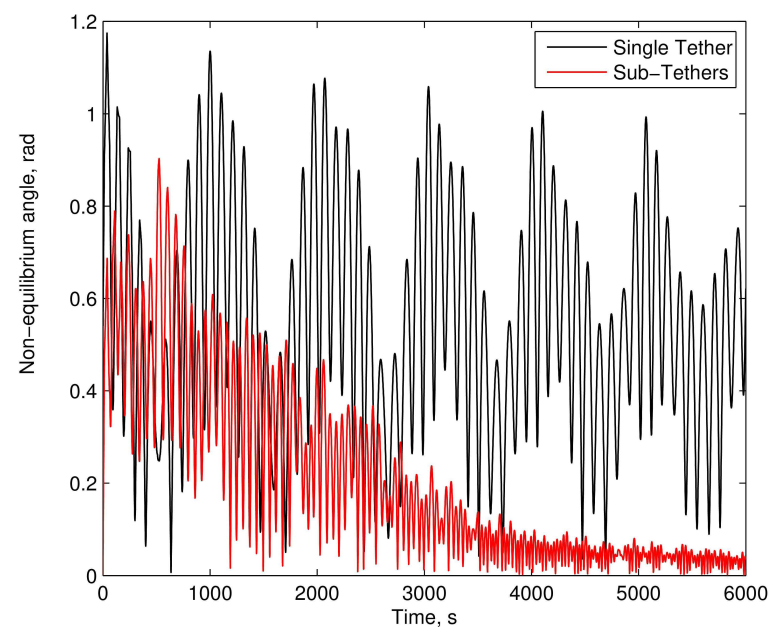

(b) Tether angle

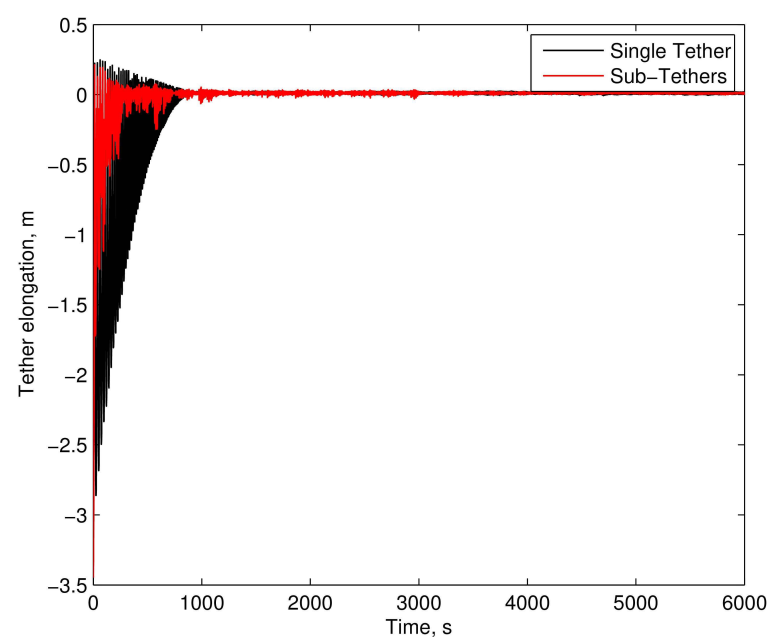

(c) Tether elongation

Figure 2.8: Resulting on-orbit TSS motion for Case 5

\subsubsection{Case 6}

Case 6 presents a different approach of controlling the attitude of the target without the use of thrust. Instead, the chaser is given an additional radial velocity of $2 \mathrm{~m} / \mathrm{s}$ such that the TSS rotates about its centre of mass. The tethers are initially tight, and there is no initial tether angle. This demonstrates another mode for preventing collision and maintaining tension in the tethers sufficient for controlling the attitude of the debris. That is, to rotate the chaser and the debris around each other (i.e., rotating the TSS about its barycentre). Figure 2.9(b) shows how after one orbit the 
tether angle in the sub-tether configuration quickly stabilizes such that the target is very close to its equilibrium attitude. The single tether configuration has angles after one orbit of 2 radians, indicating very little control of the attitude of the target. The TSS is rotating about its barycentre, so instead of reducing the target angular rates to zero, they will be non-zero and predictable. The sub-tether configuration arrives at its steady state in approximately half an orbit, whereas the single tether configuration will require many more orbits before the barycentre rotation technique has stabilized the target.

As shown in Fig. 2.9(a), this case demonstrates another stable mode of motion after capture. That is, a controlled rotation rate along two axes (rather than attempting to bring them to near zero). This technique passively yields predictable target attitude motion, once the TSS spin has been initiated. Safe separation of both configurations is demonstrated in Fig. 2.9(c).

A significant advantage of this control technique is that it does not require any thrust other than that needed to initiate the spin. This may reduce the complexity of the angular momentum dissipation process.

\subsubsection{Case 7}

This case investigates the motion of the system when the target is already stabilized and the chaser is not using thrust. This simulation provides a sense of the natural tendencies of the system, and is useful when considering the long-term safety of the debris removal system. The simulated target has initially no angular rates or tether angle, the tethers are initially tight, and the chaser and target are both passive. The simulation was performed over two orbits (12000 seconds). Simulation results, in Fig. 2.10, show that the relative motion between the chaser and the target that is induced by the orbital motion does not sustain enough tension in the tethers to maintain attitude control of the target. Gravity-gradient perturbations are dominant and control of the target is lost. Tethers can possibly become tangled in this situation. The tethers became 15 meters slack, which indicates that a collision is possible. Neither TSS configuration maintained control of the target during this simulation, demonstrating the need for active control on the chaser at all times. 


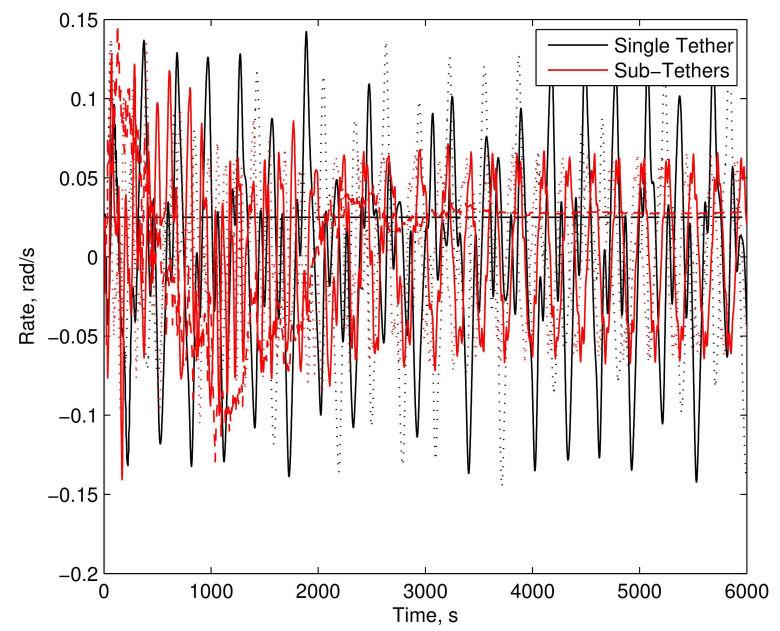

(a) Target angular rates

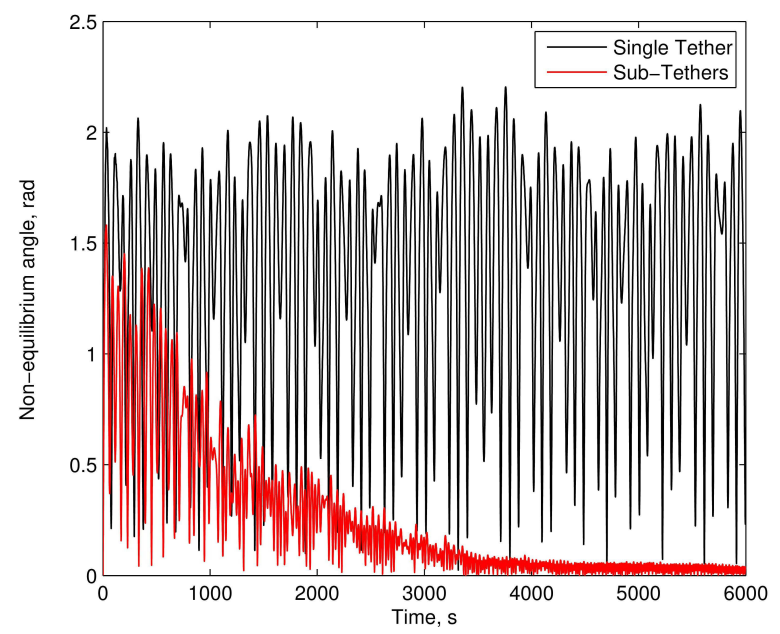

(b) Tether angle

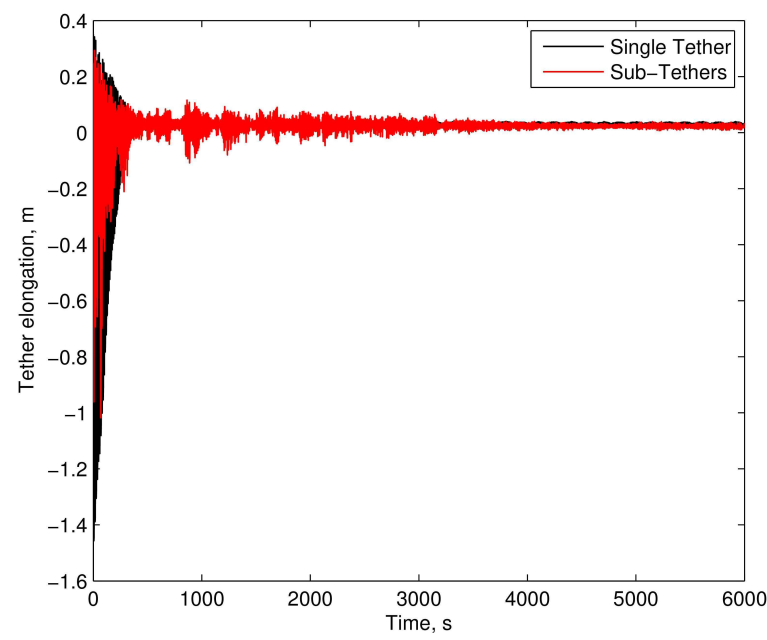

(c) Tether elongation

Figure 2.9: Resulting on-orbit TSS motion for Case 6

\subsection{Conclusions}

A novel Tethered Satellite System (TSS) where a main tether attached to the chaser branches into four sub-tethers that are attached to a tumbling passive satellite was proposed, analyzed and compared to the previously-accepted TSS configuration that has a single tether joining the two spacecraft. An orbital environment was simulated, that included orbital motion and gravity-gradient torques. Seven simulation cases were performed, each demonstrating a different capture scenario, for both the subtether TSS and the single tether TSS. 


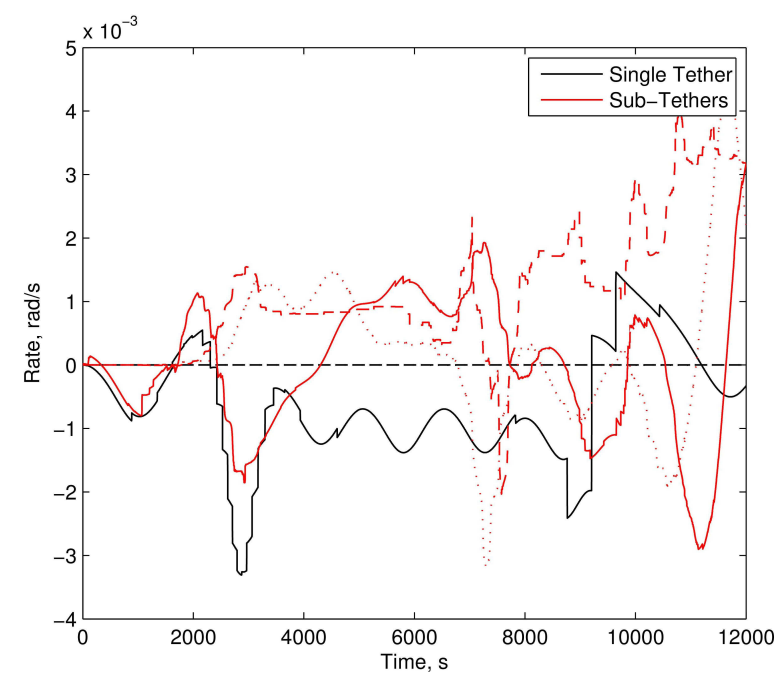

(a) Target angular rates

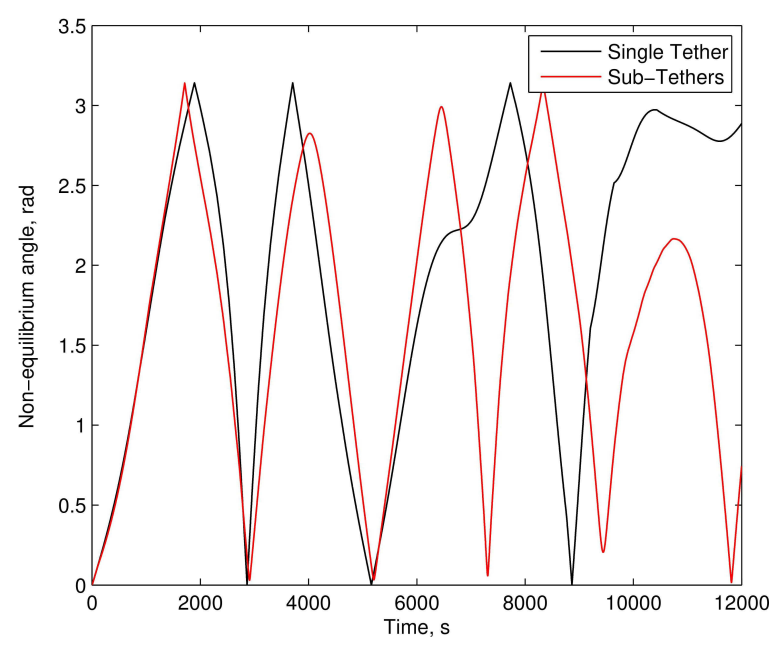

(b) Tether angle

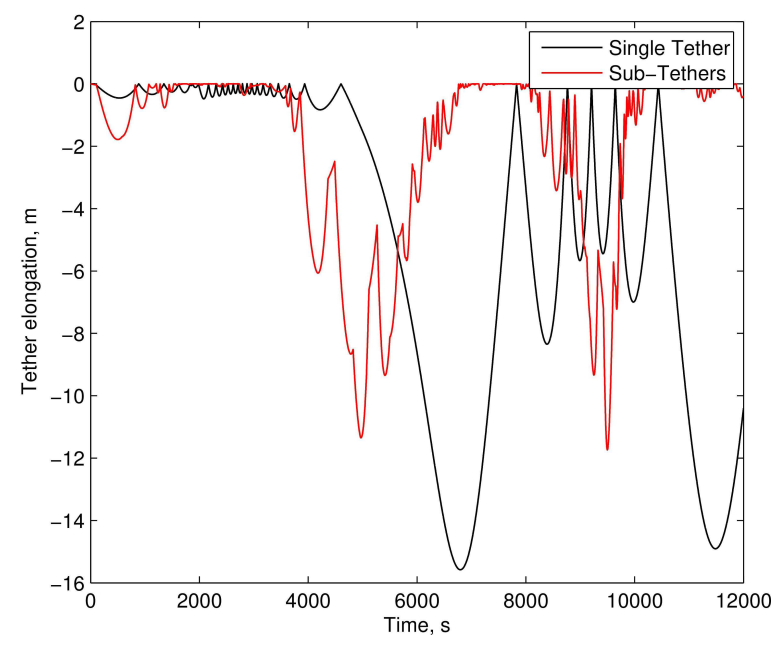

(c) Tether elongation

Figure 2.10: Resulting on-orbit TSS motion for Case 7

Cases 1-5 used thrust on the chaser spacecraft to thrust in the opposite direction to the target. In these scenarios, the thrust maintained sufficient tension in the tethers to prevent collision and regulate the angular momentum of the target. After one orbit, the resulting motion of the sub-tether TSS and the single tether TSS was compared. In these five cases, the sub-tether configuration led to a nearly stabilized target (i.e., low angular rates, and a low tether angle), ideal for transporting the target to its disposal orbit. The single tether configuration, however, did not. After one orbit, 
the angular rates and tether angle were still large. Attempting to transport an unstabilized target will result in control difficulties for the chaser, and in turn, require more fuel and a more expensive mission. Cases 1-5 shows that all else being equal, the novel sub-tether TSS proposed always leads to a significantly more detumbled target than the single tether configuration. The single tether results of Cases 1, 2, and 5 are similar to the single tether results presented by Aslanov and Yudintsev [28]. Verifying simulation results was not possible due to the unavailability of all simulation parameters; however, the same single tether trends are observed as in [28].

Case 6 demonstrated a capture scenario where the TSS is spun around its barycentre. The goal was to use the centripetal force generated in the tethers to stabilize the target, rather than use thrust from the chaser to achieve the same effect. Again, the sub-tether configuration lead to a more stable target in the same amount of time than the single tether configuration. It was demonstrated that capturing a debris in this manner could quickly stabilize a target and not require any fuel, other than that to initiate the spin.

Case 7 showed that a passive chaser should be avoided, as a collision or tether tangling could readily occur for either configuration. If a passive, safe, long-term configuration is desirable, it is recommended to initiate a barycentre rotation.

An important result from all the simulation cases is the angular momentum along the main tether axis (i.e., the $\vec{B}_{y}$ axis as shown in Fig. 2.3) cannot be controlled, no matter what TSS configuration is used. This is due to the lack of a moment arm about this axis and the flexible nature of the tether material. If a mission were being designed to capture a tumbling debris, this result indicates that the debris should be approached along its axis of least angular momentum.

In order to give more credibility to the results presented in this chapter, the equations of motion above will be stripped down into planar forms, such that planar experiments can be performed and compared to planar simulation results. The experimental facility (the Spacecraft Proximity Operations Testbed) was developed over the course of the author's masters program, and the author's contributions to the facility are presented in the following chapter. 


\section{Chapter 3}

\section{Experimental Facility}

Significant portions of the Spacecraft Proximity Operations Testbed were incomplete when the author started his MASc. program. In order to experimentally validate tether detumbling simulations, the laboratory had to be completed. The author was responsible for the design, purchase, build, and commissioning of the following systems: air refill station, propulsion system, floatation system, reaction wheel system, emergency stop system, power distribution and charging system, ground truth system, data processing system, and a complete software template. The resulting planar laboratory facility allows for simulation and experimentation of spacecraft technologies.

\subsection{Introduction}

The Spacecraft Proximity Operations Testbed (SPOT) is a unique facility in the Spacecraft Robotics and Control Laboratory at Carleton University. The facility is the largest of its kind in Canada, and positions Carleton University as highly relevant in the space robotics research field.

The SPOT consists of a large granite table that is manufactured to be extremely smooth. Spacecraft platforms are placed on the table and are designed to float around on the smooth surface. In order to float on the granite surface, the platforms expel air downward through air bearings to create a thin film of air between the air bearing and the granite table. The effect is similar to a puck floating on an air hockey table, except in the SPOT the air is forced downwards from the platform instead of upwards through the table as is done in air hockey. This thin air film creates a near frictionfree planar environment that closely mimics the dynamics of deep space, allowing researchers to test real spacecraft technologies in a laboratory environment.

Upon the author's arrival to the MASc program, the granite table was installed 
but the spacecraft platforms and all accompanying technology had to be designed, built, and commissioned. The author made significant contributions to the SPOT in order to bring it to a state such that spacecraft guidance, navigation, and control experiments could be performed. Once complete, the laboratory facility was then used to test the topic of this thesis, i.e., comparing the effectiveness of the sub-tether and single tether configurations at detumbling a debris. This chapter presents the work done to bring the SPOT to an operational state.

Figure 3.1 shows an overview of the SPOT as of March 2017. It shows the granite table, the lab room, the ground station computer, and the two spacecraft platforms. Figure 3.2 shows a close up of the two spacecraft platforms that were designed and built. Three prototypes were built for testing purposes but their details will not be included in this thesis.

The spacecraft platforms are built in a modular fashion, such that they can easily be disassembled and modified. There are three main "decks" (i.e., levels), each with a specific purpose: the Sensor deck houses computer vision equipment, the Avionics deck contains the power, battery, computer, control panel, and reaction wheel systems, and the Bus deck contains the air tank, the floatation system, and the propulsion system. The three decks are stacked on top of each other, with the Bus deck at the bottom, the Avionics deck in the middle, and the Sensor deck at the top. An outer structure holds everything in place.

All three decks are wired for $12 \mathrm{~V}$ and $5 \mathrm{~V}$ power. The $5 \mathrm{~V}$ line typically powers the avionics while the $12 \mathrm{~V}$ line powers the electric motors. When floating, the platforms can use their on-board thrusters to translate around the table. A reaction wheel is also available, and can be used for attitude control instead of the thrusters, if desired. A detachable robotic arm can be used for robotic capture and docking experiments.

Throughout an experiment, a ground truth system records the platform's position and attitude and transmits this information to the platforms in real-time for use in closed-loop control systems. A Raspberry Pi 3 is used as the on-board main computer and it communicates wirelessly with the ground station computer to receive ground truth data, start and stop commands, and executable files. The software executed 
on the Pi 3 is automatically generated from the software that is used to perform numerical simulations in MATLAB/Simulink. Therefore, once the numerical simulation performs as desired, the software can then be exported for use in experiments. The platforms will then execute the same logic present in the numerical simulation.

The only consumables are the air in the air tank and the charge of the battery. A cutout on the side of the platform allows the user to easily swap out an empty air tank for a full one, and a charging port on the control panel allows for the battery to be charged without removing it. A main power switch and status LEDs are located on the control panel on each platform. An emergency stop button on the ground station table cuts power to the $12 \mathrm{~V}$ line (i.e., all of the mechanical components). Two spacecraft platforms (a red and a black one) are built identically.



Figure 3.1: Overview of the Spacecraft Proximity Operations Testbed.

Section 3.2 provides high-level details about all of the laboratory components the author contributed to the SPOT, of which all the technical details can be found in Appendix A (intended for future students). Section 3.3 presents the specific experimental setup the author used to perform the tether detumbling experiments relevant to the author's research. 


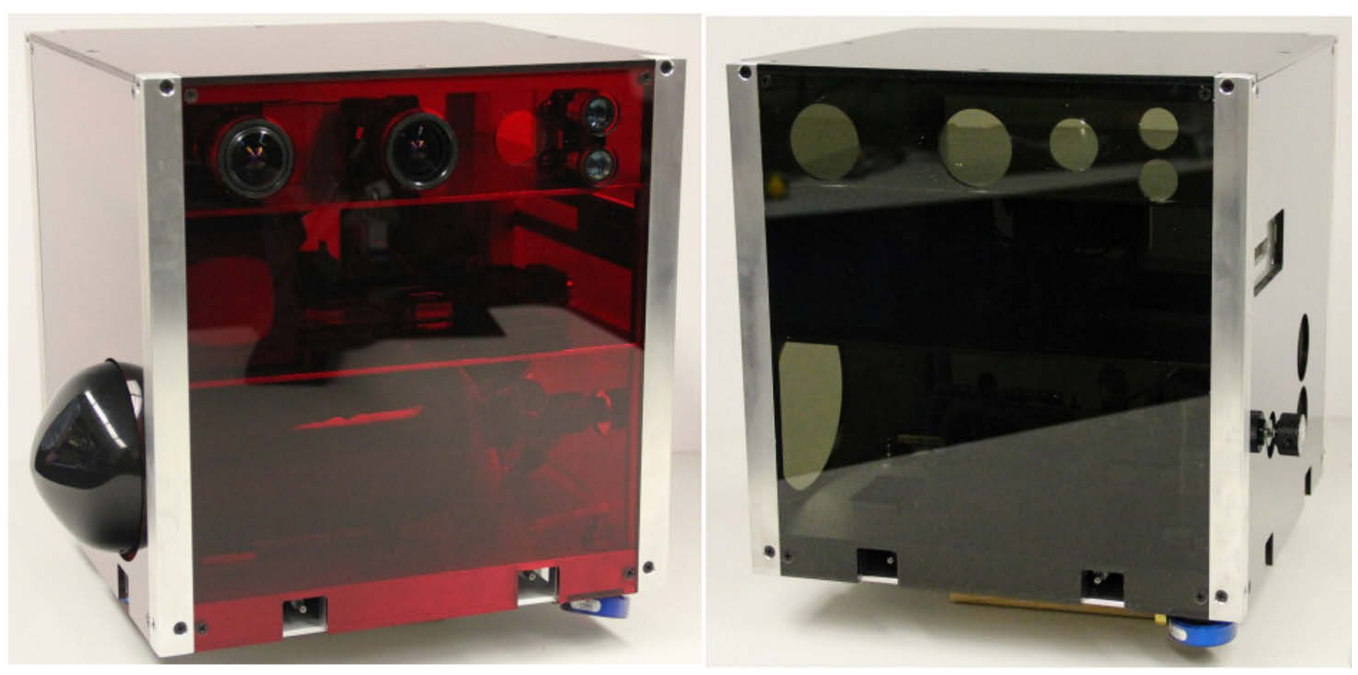

Figure 3.2: Spacecraft platforms built for the SPOT.

\subsection{Laboratory Contributions}

This section presents overviews of the major systems that the author designed and built for use in the SPOT.

\subsubsection{Floatation System}

Three $40 \mathrm{~mm}$ round New Way Air Bearings were used to provide floatation to each platform. A top and bottom view of an air bearing is shown in Fig. 3.3.

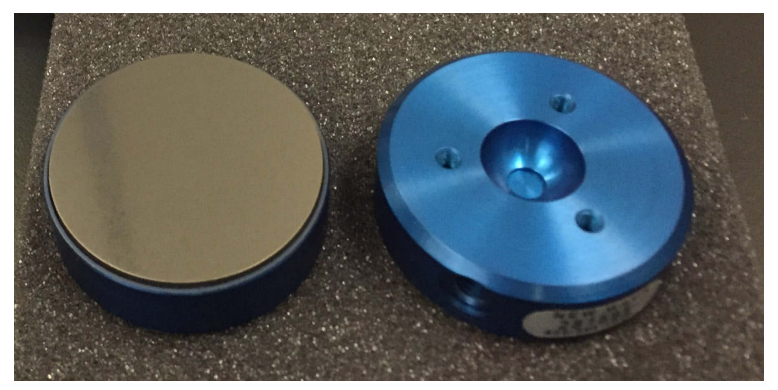

Figure 3.3: Bottom of air bearing is shown on the left. The top of the bearing and mounting holes are shown on the right.

Air is fed to the air bearings through flexible PVC hosing at 60 psi, which causes the air bearings to hover around 5 microns above the surface of the granite table. A schematic of the air flow is shown in Fig. 3.4. 


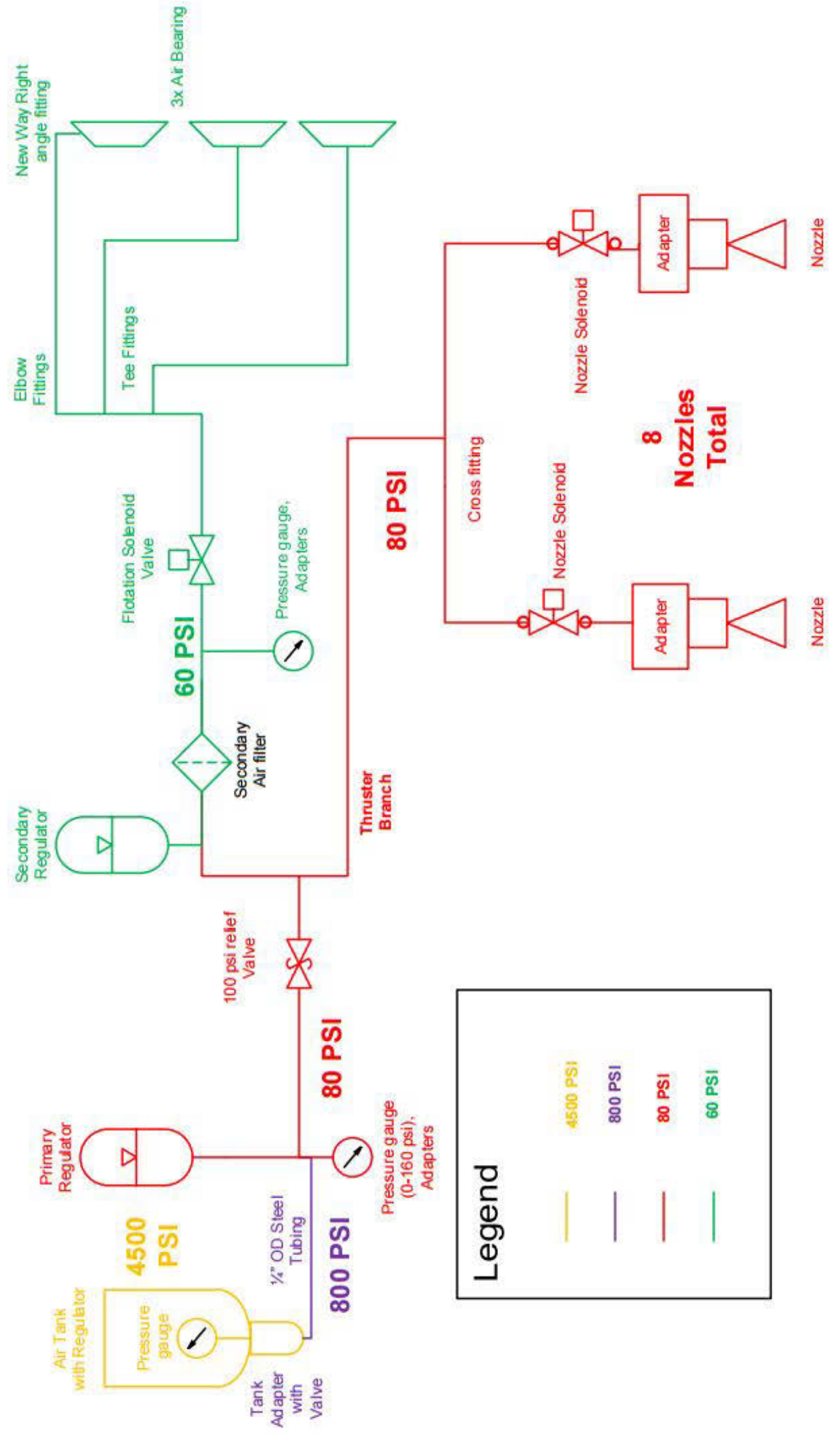

Figure 3.4: Air flow diagram responsible for the on board propulsion and floatation systems. Only two thrusters are shown for clarity. 
The yellow air tank, depicted in Fig. 3.4, stores compressed air at 4500 psi. A regulator built into the air tank reduces the output pressure to 800 psi. The air flows into the High Pressure Regulator, that regulates the pressure to 80 psi. The 80 psi air is fed to the air nozzles that provide thrust, and to a low pressure regulator that brings the pressure to $60 \mathrm{psi}$ which is suitable for the air bearings. The air is filtered before reaching the air bearings to remove any contaminants that may have entered the air supply. A pressure relief valve is included for safety, and will automatically vent the air from the lines if the high pressure regulator fails and $>100 \mathrm{psi}$ is experienced in any of the downstream lines. Numerous fittings are used to join the tubing to all the components.

\subsubsection{Propulsion System}

The propulsion system is responsible for the translation and attitude control of the spacecraft platform. Air is delivered to the 8 thrusters and is passed through nozzles to produce thrust, as shown in Fig. 3.4. At 80 psig the nozzles are rated to produce $1.0 \mathrm{~N}$ of thrust. The implementation of the air delivery and propulsion systems is shown in Figs. 3.5 and 3.6.

The opening and closing of the thrusters is controlled by normally-closed solenoid valves. By applying $12 \mathrm{~V}$ of direct current, the valves open in less than $10 \mathrm{~ms}$. The solenoid valves are driven by power MOSFETs, which are controlled from the on-board computer. By intelligently opening and closing the thruster valves, any desired force and torque can be achieved, within the limits of the actuators. A control mixer was developed, that converts the desired force and torque requested by the controller into individual thruster duty cycles. Refer to the thruster numbering shown in Fig. 3.7.

Consider the thrust distribution matrix, $\mathbf{H}$, below:

$$
\mathbf{H}=2 F_{\text {thrust }}\left[\begin{array}{cccccccc}
-1 & -1 & 0 & 0 & 1 & 1 & 0 & 0 \\
0 & 0 & 1 & 1 & 0 & 0 & -1 & -1 \\
d_{1} & -d_{2} & d_{3} & -d_{4} & d_{5} & -d_{6} & d_{7} & -d_{8}
\end{array}\right]
$$

where $d_{i} \forall i=1, \ldots, 8$ are the thruster distances from the centre of mass, and $F_{\text {thrust }}$ 


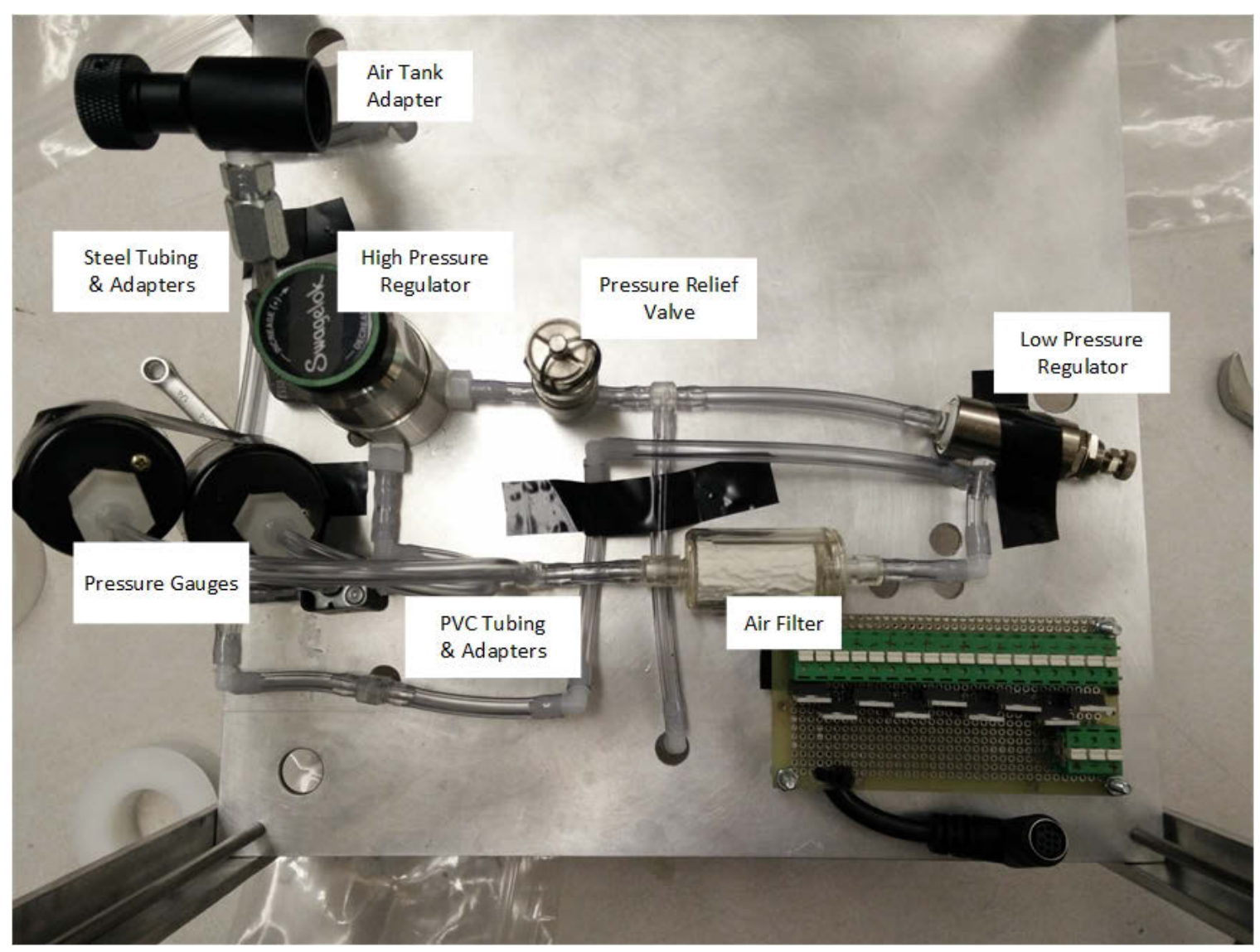

Figure 3.5: Air flow on the top side of the bus deck. Here, the air passes through regulators before being divided to the thrusters and the air bearings.

is the force developed by any thruster. The first row of the $\mathbf{H}$ matrix represents the amount each thruster contributes to the force on the platform in the body-fixed $\vec{T}_{x}$ direction. The second row similarly represents the forces contributed by each thruster in the $\vec{T}_{y}$ direction. The third row represents the torques applied by each thruster to the platform. Also consider the thruster state matrix $\mathbf{u}$, defined by:

$$
\mathbf{u}=\left[\begin{array}{llllllll}
\hat{u}_{1} & \hat{u}_{2} & \hat{u}_{3} & \hat{u}_{4} & \hat{u}_{5} & \hat{u}_{6} & \hat{u}_{7} & \hat{u}_{8}
\end{array}\right]^{\mathrm{T}}
$$

where $\hat{u}_{i} \forall i=1, \ldots, 8$ is the duty cycle for each thruster (i.e., a value of 0.5 means the thruster is on for $50 \%$ of the time). 


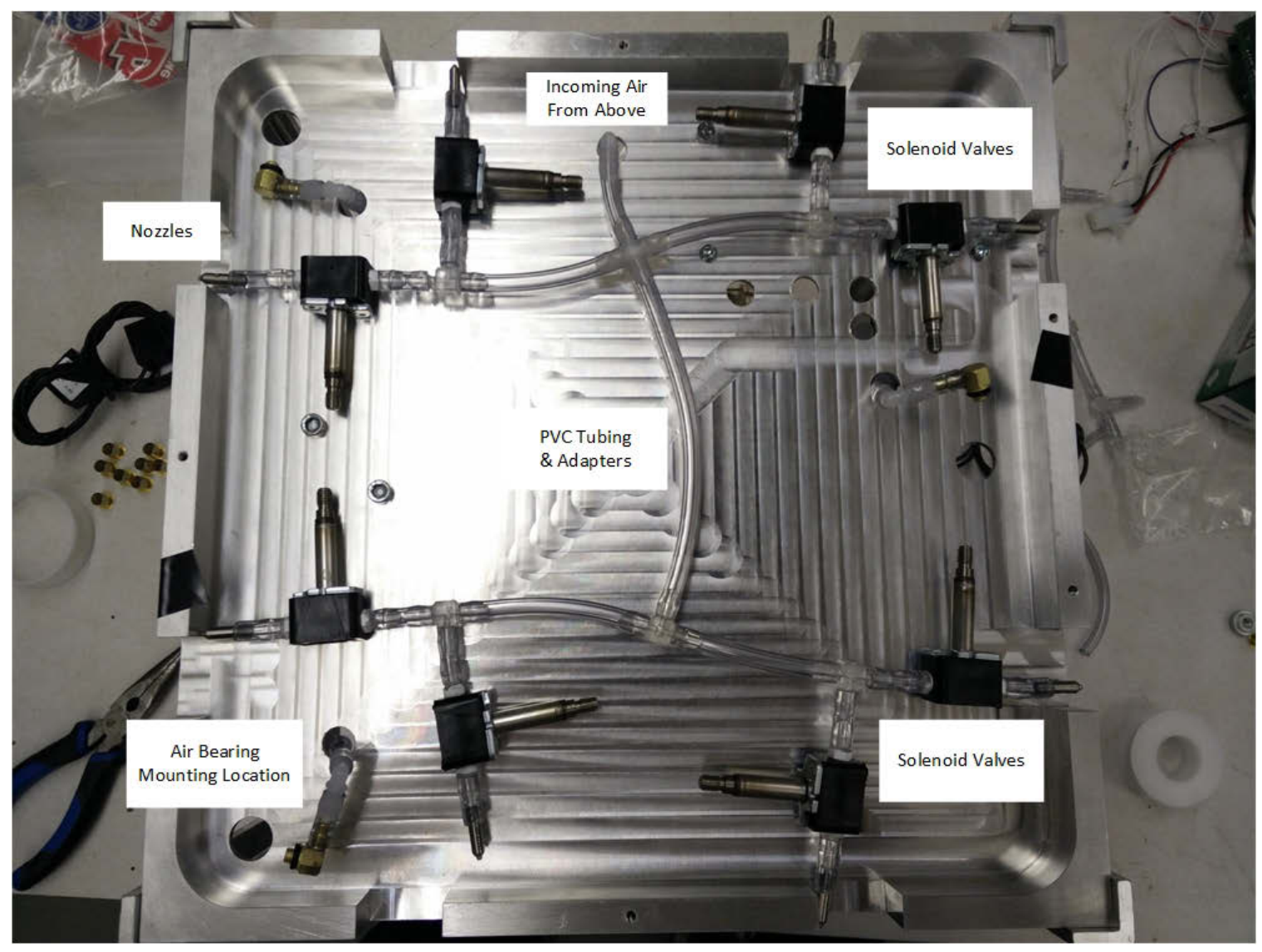

Figure 3.6: Air flow on the underside of the bus deck. Here, the air is delivered to the 8 thrusters and 3 air bearings.

The generated force and torque are, in the body-fixed reference frame:

$$
\left[\begin{array}{c}
F_{x}^{t} \\
F_{x}^{t} \\
\tau^{t}
\end{array}\right]=\mathbf{H u}
$$

where $F_{x}^{t}$ and $F_{y}^{t}$ are the generated forces in the body-fixed frame in the $\vec{T}_{x}$ and $\vec{T}_{y}$ directions, respectively, and $\tau^{t}$ is the torque developed. Therefore, for any commanded $F_{x}^{t}, F_{y}^{t}$, and $\tau^{t}$, the ideal thruster duty cycles can be found through:

$$
\mathbf{u}=\mathbf{H}^{\mathrm{T}}\left(\mathbf{H H}^{\mathrm{T}}\right)^{-1}\left[\begin{array}{c}
F_{x}^{t} \\
F_{x}^{t} \\
\tau^{t}
\end{array}\right]
$$




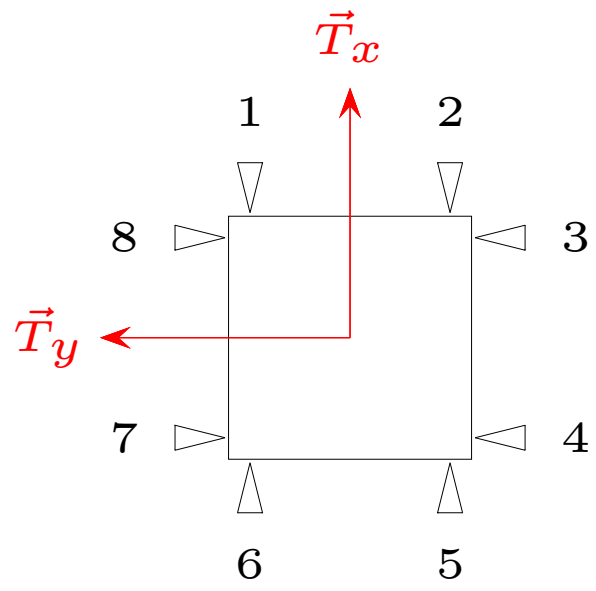

Figure 3.7: Thruster numbers and body-fixed reference frame on each spacecraft platform.

which is a least squares estimate since $\mathbf{H}^{-1}$ does not exist. The resultant $\mathbf{u}$ is a series of thruster duty cycles. Typically, half of the ideal duty cycles are negative, and are discarded (i.e., set to zero), since negative thrust is mathematically but not physically possible. The factor of 2 is present in Eq. (3.1) to double the duty cycles for the thrusters that are not discarded.

Once the thruster duty cycles are obtained, the thrusters are actuated using pulsewidth-modulation with a period of 0.1 seconds.

Equation (3.1) requires the knowledge of the location of the thrusters with respect to the centre of mass of the platform. This allows the control mixer to know the torque that each thruster can provide to the platform. The thruster distances with respect to the centre of mass are not readily available, so a simple technique was developed to determine the location of the centre of mass of the platform. By floating a platform, spinning it, and releasing, it is known that the platform will spin about its centre of mass. Therefore, by observing the position of the four PhaseSpace LEDs (discussed in Sec. 3.2.7) the resulting LED position with respect to the centre of mass can be obtained. By measuring the thruster positions with respect to the LEDs, the thruster distances from the centre of mass can be obtained. In addition, the slope in the granite 
table can be characterized. The calibration can easily be repeated whenever changes to the platform are performed that change the location of the centre of mass.

It is acknowledged that there are some flaws in the current propulsion system. For example, it is unlikely that all thrusters produce the same amount of thrust. Furthermore, since all thrusters have different distances from the centre of mass of the platform, simply rejecting any negative thruster duty cycles and doubling the positive ones does not yield the desired force and torque. An improved control mixer that iteratively solves for the proper duty cycle is in development.

\subsubsection{Air Refill Station}

The air tanks used on the spacecraft platforms are filled to 4500 psig using the Air Refill Station. The air bearings drive the quality of the air that needs to be stored in the air tanks. The air bearings require dry, clean, air of ISO Class 3 or better. Specifically, particles larger than 5 microns must be filtered out, the air must be dried to have a dewpoint of $-4 \mathrm{~F}$ at $100 \mathrm{psig}$, and there must be less than $1.0 \mathrm{mg} / \mathrm{m}^{3}$ of oils present.

Figure 3.8 shows the refill station. The gray air compressor at the bottom of the figure is the Omega Supercharger, which has the capability to pressurize the ambient air to 4500 psig. The output air from the compressor is dried by passing the air through the red air dryer on the left. The air dryer is filled with a desiccant that absorbs the moisture from the air. Its performance parameters are unknown but assumed to be sufficient. Next, the air passes through the blue air filter. This filters out particles larger than 0.1 microns in size, and removes oil vapours down to 0.03 $\mathrm{mg} / \mathrm{m}^{3}$. The dried, cleaned, air is then fed into the air tank. The air tank is typically used for air guns. The system takes around 1 hour and 45 minutes to completely fill a depleted air tank.

\subsubsection{Reaction Wheel}

To perform attitude manoeuvres on the spacecraft, a reaction wheel is optionally available, as an alternative to using the thrusters for attitude control purposes. A reaction wheel consists of a metal disc that is spun about its axis of symmetry. 


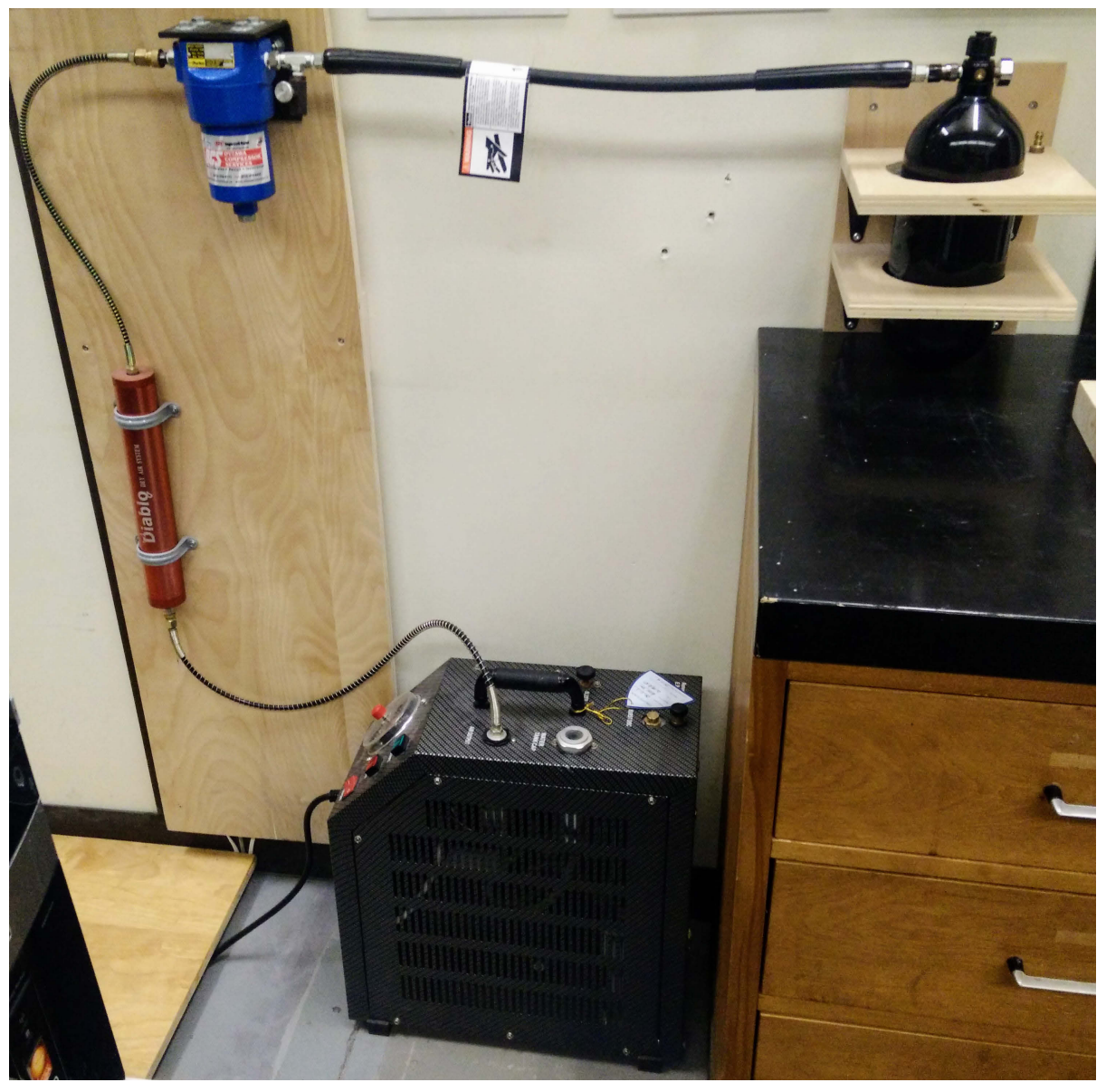

Figure 3.8: Air refill station. 


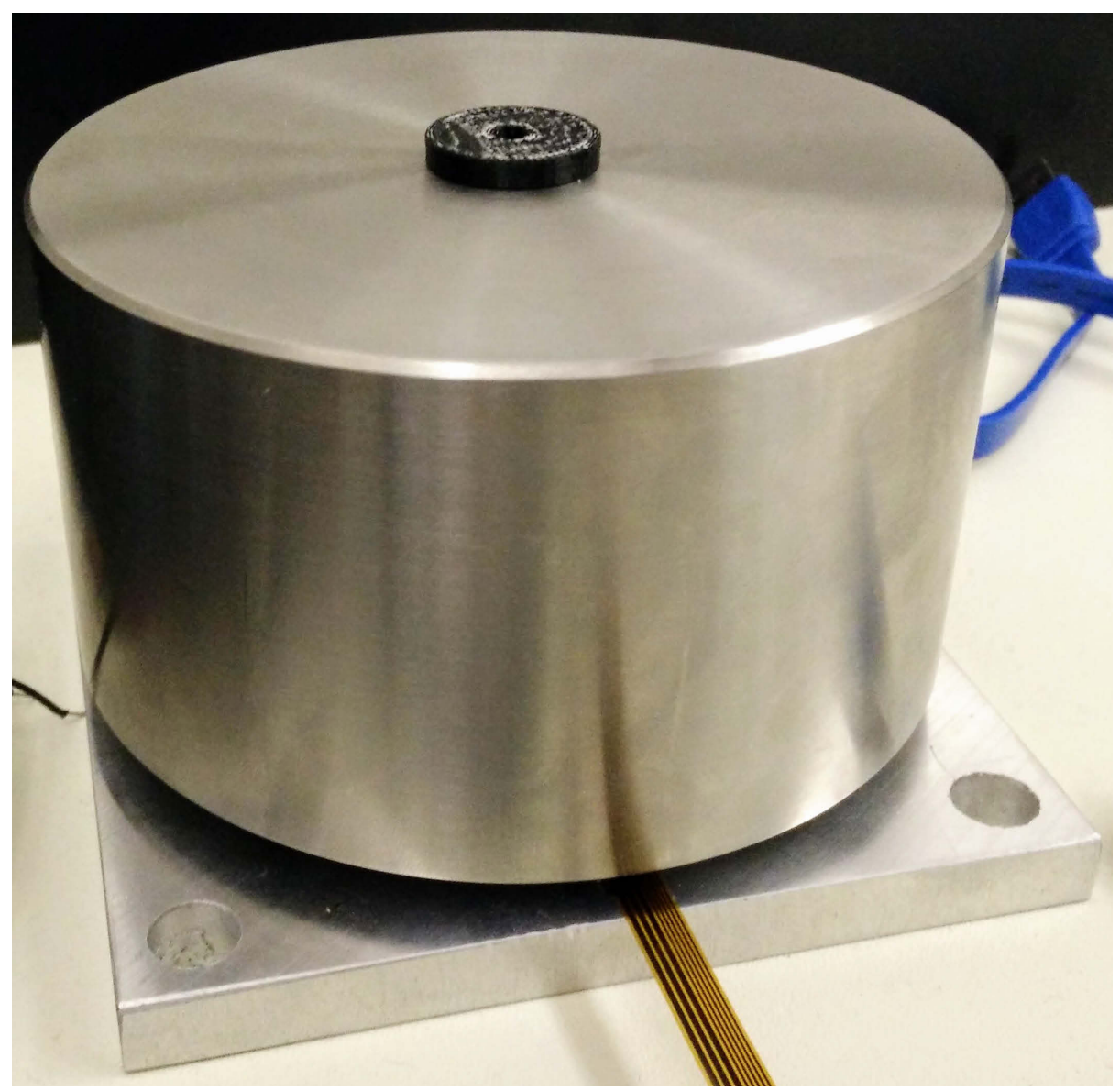

Figure 3.9: Reaction wheel.

When the rotational speed of the wheel is increased, an equal and opposite torque is imparted to the spacecraft. Reaction wheels consume electricity to perform attitude manoeuvres instead of fuel. Therefore, reaction wheels are often used for attitude control of spacecraft. A reaction wheel is useful for the SPOT because it allows for the thrusters to only be used for translational motion.

Figure 3.9 shows the reaction wheel that was designed and built for the SPOT. A stainless steel wheel was machined from a solid cylinder, and is the prominent item in the figure. The wheel spins on a thrust bearing, such that the weight of the wheel is transmitted through the aluminium base plate. A brushless motor and gearbox are placed underneath the centre of the wheel, such that they can provide a torque to spin the reaction wheel but not bear the weight of the wheel. Therefore, by changing the wheel speed as a function of time, any desired torque can be achieved. The on 
board computer passes the desired wheel speed to a speed controller, that performs closed-loop feedback control to achieve the desired wheel speed. Closed-loop feedback is provided by Hall effect sensors on the motor.

The reaction wheel has an inertia of $0.0074 \mathrm{~kg} \cdot \mathrm{m}^{2}$ and a maximum angular velocity of 128 RPM, which leads to a maximum angular momentum storage of 0.1 Nms. This yields a maximum angular rate imparted to the spacecraft platform of 19 degrees per second.

\subsubsection{Power and Computer System}

A power system was designed to deliver 5 and 12 Volts of power to the onboard electronics. A rechargeable 24 Volt battery is fed, in parallel, to $12 \mathrm{~V}$ and $5 \mathrm{~V}$ voltage regulators. Both power lines share the same ground, and are tied to the negative terminal on the battery. Figure 3.10 shows an electrical diagram of the system, and Fig. 3.11 shows the avionics deck where the power and computer system are physically located.

A voltage divider present on the $24 \mathrm{~V}$ lines allows the present voltage output of the battery to be measured by the Arduino Mini. As the battery depletes, the voltage will drop. When the battery is below a certain threshold (23.8 V), the Arduino Mini triggers a low battery LED to alert the operator. The Arduino Mini also controls the Emergency Stop Circuitry, which is explained further in Sec. 3.2.6.

A Raspberry Pi 3 is used as the main computer. The output pins are used to command the air bearings, thrusters, reaction wheel, and other components. Interfacing the software with the Pi 3 pin states was performed by another student.

\subsubsection{Emergency Stop System}

It is desirable to be able to shut down the spacecraft platforms in an emergency situation. For this reason, a standalone wireless emergency stop was constructed. It is responsible for cutting power to the $12 \mathrm{~V}$ power supply on board the spacecraft platforms in order to stop the thrusters, reaction wheel, robotic arm, and any future systems that have the potential to cause harm to the platforms. However, it is important to not cut power to the air bearings, to avoid damaging the air bearings 


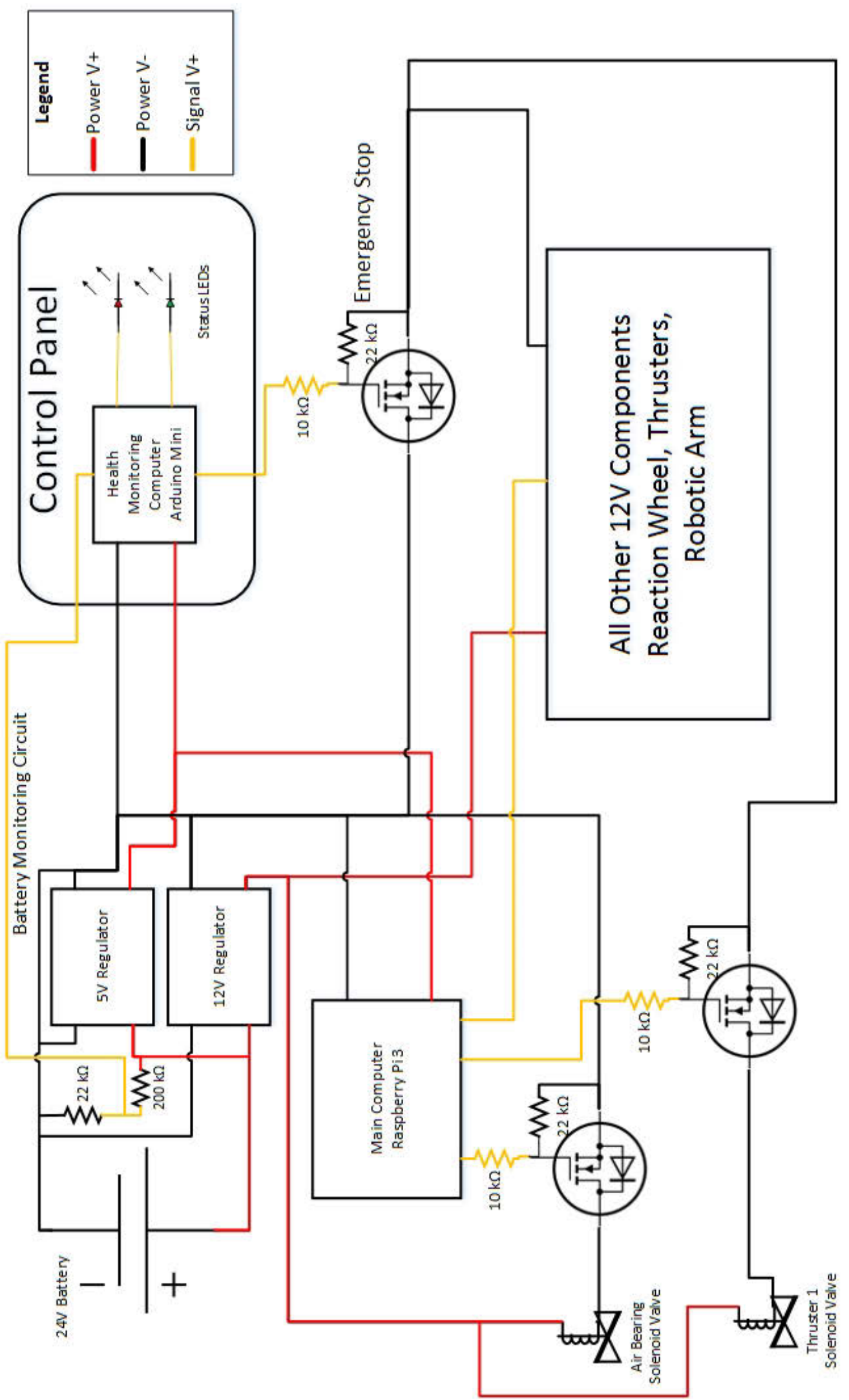

Figure 3.10: Electrical schematic present on the avionics deck of the spacecraft platforms. 


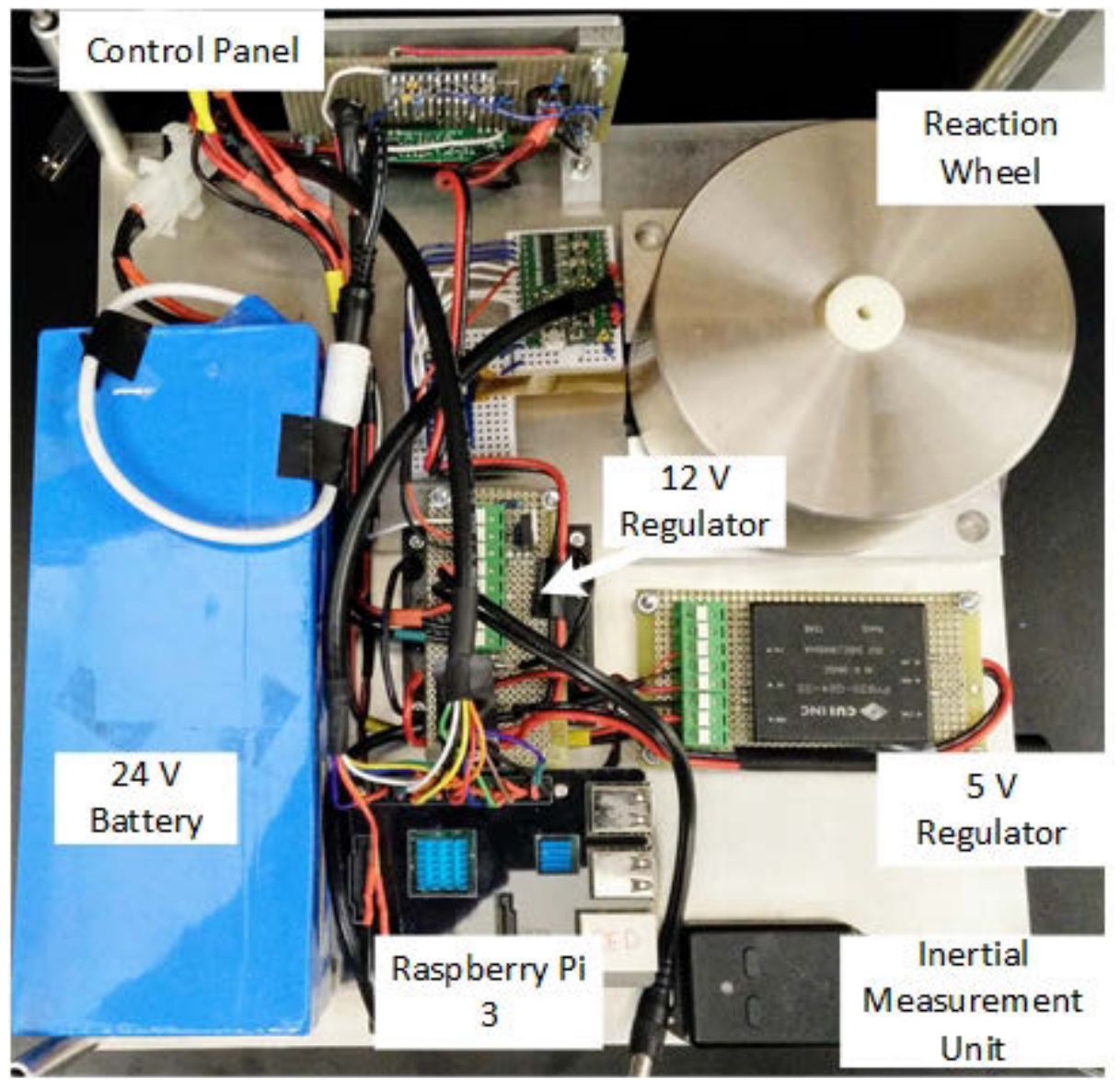

Figure 3.11: Avionics deck on board the spacecraft platforms. This deck contains the power subsystem, the on board computer, the reaction wheel, and the control panel. 
in the event that the platform has a high velocity before the power was cut. This poses an additional risk that the platforms may fall off the table in an emergency, but will be mitigated by having experimenters near the table at all times. The $5 \mathrm{~V}$ power line should remain on at all times to allow for the computers to be powered on for communication purposes.

The emergency stop circuitry is shown in Fig. 3.10. All components on the $12 \mathrm{~V}$ power line pass through the Emergency Stop MOSFET except for the air bearing solenoid. The Arduino Mini receives a wireless signal (on a separate channel than the main communications link) that indicates whether or not to engage or disengage the emergency stop. The emergency stop is fail safe, i.e., if no signal is received for 5 seconds, the emergency stop is automatically triggered.

\subsubsection{Ground Truth System}

While experiments are in progress, it is necessary to collect and record position and attitude data. A PhaseSpace system was acquired for this task. Eight cameras were installed and pointed towards the granite table in the SPOT. Four active LED markers are placed on the spacecraft platforms and are illuminated during experiments. When illuminated, the cameras track the positions of the LEDs. Knowing where the LEDs are located with respect to the centre of mass of the platform, the position of the centre of mass and the attitude can be calculated. The PhaseSpace server performs this calculation and returns the position and attitude data at $240 \mathrm{~Hz}$ to the ground station computer. The data is logged for analysis after the experiment. The spacecraft platform position and attitude data is wirelessly sent to the platforms in real time at $20 \mathrm{~Hz}$ for use in feedback control systems, if necessary.

\subsubsection{Software}

A significant amount of software was developed to program, compile, and upload software to the spacecraft Raspberry Pi 3 computer, communicate wirelessly with the Pi 3, collect, record, and process data from the PhaseSpace system, perform numerical simulations, and calibrate the spacecraft platforms. The goal was to design software that is easy for researchers who are unfamiliar with the fine details of lab. 
The software flow is as follows:

- A user makes a copy of the software Template (made in MATLAB/Simulink)

- The user inputs a desired initial position of the spacecraft platform into the accompanying MATLAB code and adds in the logic of their experiment. The platform will move to the desired initial position, perform the desired logic, and then return to the "home" position automatically.

- The user performs simulations by running a "runSimulation.m" script. This models the dynamics of the spacecraft platforms, and the resulting motion is animated when the simulation is complete.

- Once the simulated spacecraft platforms perform as desired, the user clicks "Export" which outputs the MATLAB/Simulink code in a form that can be run on the on-board computers in an experiment. No changes are required in the code between simulation and experiment.

- The output software is automatically compiled and uploaded to the spacecraft platforms by the "AutoCompile" software.

- The user then issues a "Run" command, which triggers the recording of PhaseSpace data and initiates the experiment. The spacecraft performs the experiment.

- The desired data is collected and stored on the ground station computer.

- The user runs the "Compare Data.m" script, which plots the position and attitude data for both the simulation and the experiment for easy comparison. This software can be modified to plot parameters specific to the researcher's needs.

Using the same software for simulation and experiment allows for an extremely short amount of time needed to go from a simulation to experiment, and eliminates the possibility of coding errors when transcribing from a simulation to an experiment. The Template software allows for an idea to be brought to a simulation without having to worry about the fine details and logistics of the facility. 
Full details about all the contributions to the Spacecraft Proximity Operations Testbed can be found in Appendix A. The specific setup of the lab in order for tether experiments to be performed is detailed in the following section.

\subsection{Experimental Setup}

Section 3.2 presented the contributions the author made to the Spacecraft Proximity Operations Testbed. This section presents how the experimental facility was setup for debris detumbling experiments.

Two spacecraft platforms were used, along with the accompanying systems discussed in Sec. 3.2. The thrusters were used for attitude control instead of the reaction wheel. The RED platform, shown in Fig. 3.2, was chosen to act as the "Chaser" and the BLACK platform was chosen to act as the "Uncooperative debris". A tether was purchased and attached to both platforms. Both a single tether configuration and a sub-tether configuration were used. The following subsections discuss the characterization of the tether material used and the position and attitude controllers that were implemented.

\subsubsection{Tether Model}

A braided elastic tether material consisting of $56 \%$ polyester and $44 \%$ rubber was obtained for use in experiments. A flexible tether material with a low stiffness was chosen to allow for measurable tether stretches with short tethers in a laboratory setting. By suspending known masses from a tether segment and measuring its resulting stretch, it was found that the tether material has a non-linear stiffness. The three sub-tether segments are identical in length and their stiffness model is shown in Fig. 3.12(a). The single tether stiffness model is shown in Fig. 3.12(b). For a given tether stretch, the corresponding stiffness in the tether is found by interpolating the experimental data using the spline function in MATLAB/Simulink.

Since a reliable test to determine the tether damping was not available, the tether damping coefficient was assumed to be constant. To determine the tether damping constant, a preliminary set of detumbling experiments were performed. Then, simulations were ran with different tether damping constants until the simulations and 


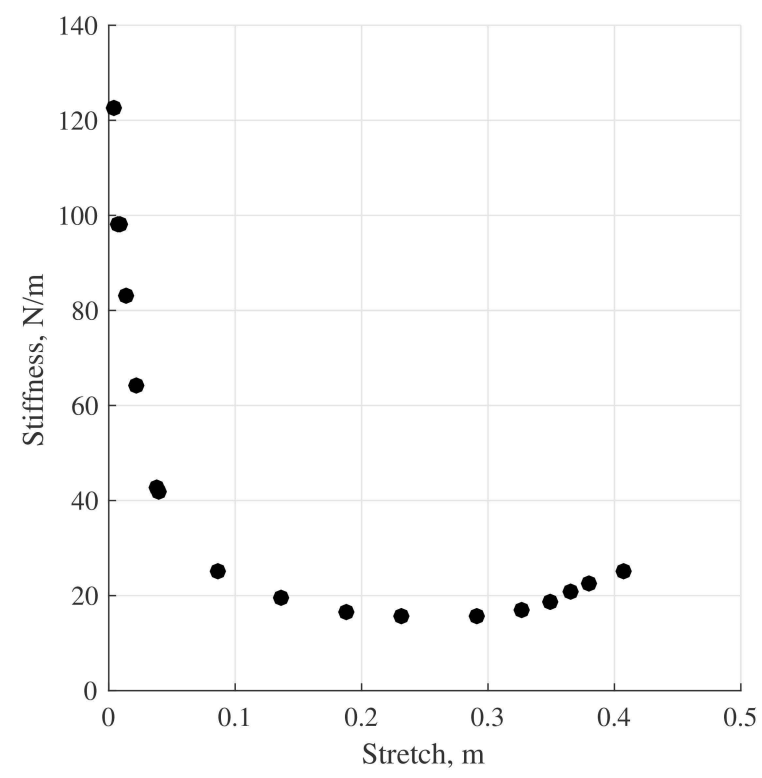

(a) Sub-tether

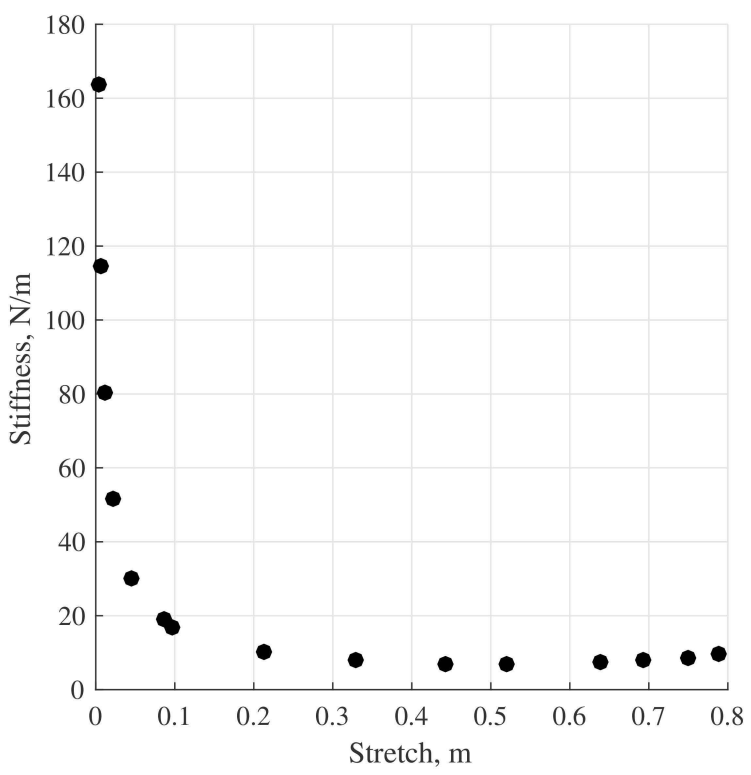

(b) Single tether

Figure 3.12: Experimental non-linear tether stiffness model, generated by hanging known masses from each tether and measuring the resulting stretch.

experimental results showed a similar decay rate. The damping coefficient primarily affects the decay rate of the resulting motion. Then, using the determined tether damping constants, the final simulations and experiments presented in Chapters 4 and 5 were performed.

Figure 3.13 shows the two spacecraft platforms tethered together on the granite table in the sub-tether configuration.

A simple proportional plus derivative controller is used for position and attitude control where needed, and is discussed in Sec. 3.3.2.

\subsubsection{Controller Design}

A simple proportional plus derivative (PD) controller is used to generate the appropriate $\mathbf{F}_{\text {control }}$ and $\tau_{\text {control }}$ for the chaser spacecraft. Consider the block diagram of the control scheme in Fig. 3.14. The closed-loop transfer function for the system is

$$
\frac{\mathbf{r}_{c}(s)}{\mathbf{r}_{d e s}(s)}=\frac{\frac{k_{p}}{m}}{s^{2}+\frac{k_{d}}{m} s+\frac{k_{p}}{m}}
$$




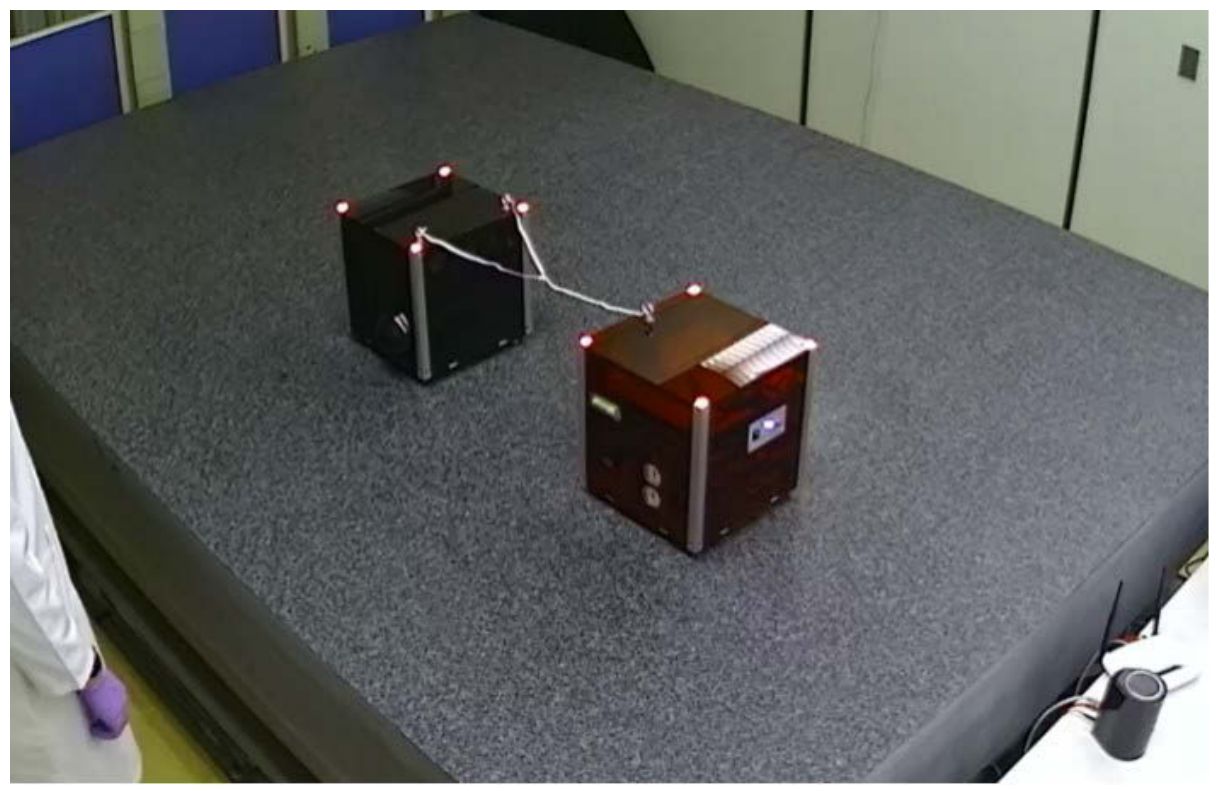

Figure 3.13: RED chaser tethered to the uncooperative BLACK debris on the granite table in Carleton's SPOT.

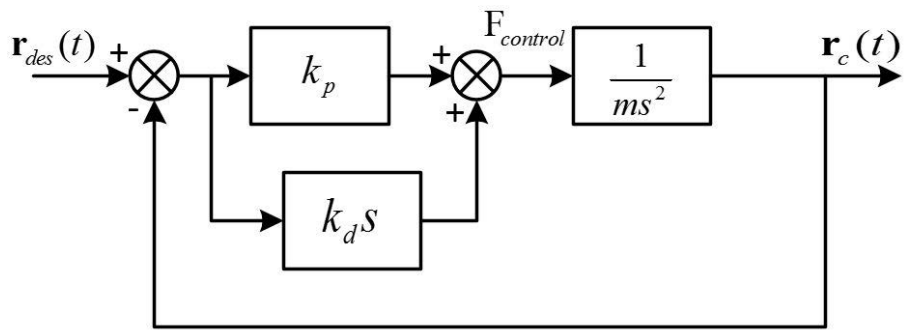

Figure 3.14: Block diagram of the $\mathrm{PD}$ controller for translational control, where $\mathbf{r}_{\text {des }}(t)$ is the desired position and $\mathbf{r}_{c}(t)$ is the actual position.

where $k_{p}$ and $k_{d}$ are the proportional and derivative gains of the control system, respectively. A standard second-order system takes the form

$$
\frac{Y(s)}{X(s)}=\frac{\omega_{n}^{2}}{s^{2}+2 \zeta \omega_{n}+\omega_{n}^{2}}
$$

where $\omega_{n}$ and $\zeta$ are the desired natural frequency and damping ratio of the closed-loop system, respectively. By inspection, it is found that

$$
\begin{aligned}
& k_{p}=\omega_{n}^{2} m \\
& k_{d}=2 \zeta \omega_{n} m .
\end{aligned}
$$


To calculate the control gains $k_{p}$ and $k_{d}$, the time domain settling time specification, $t_{s}$, is used. The settling time is the time it takes for the system to reach and remain within $2 \%$ of the desired value when a step input is applied without disturbances. The natural frequency, $\omega_{n}$, is calculated from the damping ratio and the settling time, through [45]:

$$
\omega_{n}=\frac{\ln \left(0.02 \sqrt{1-\zeta^{2}}\right)}{-\zeta t_{s}} .
$$

With the damping ratio and natural frequency selected, the control gains $k_{p}$ and $k_{d}$ can be found using Eqs. (3.7) and (3.8) and finally the control actuation in $\mathcal{F}_{I}$ is obtained through

$$
\mathbf{F}_{\text {control }}=k_{p} \mathbf{e}+k_{d} \dot{\mathbf{e}}
$$

where $\mathbf{e}$ is the position error signal in $\mathcal{F}_{I}$, i.e.,

$$
\mathbf{e}=\mathbf{r}_{d e s}(t)-\mathbf{r}_{c}(t)
$$

For rotational control, a very similar PD controller is designed. Figure 3.15 shows a block diagram of the controller. The plant dynamics include the moment of inertia

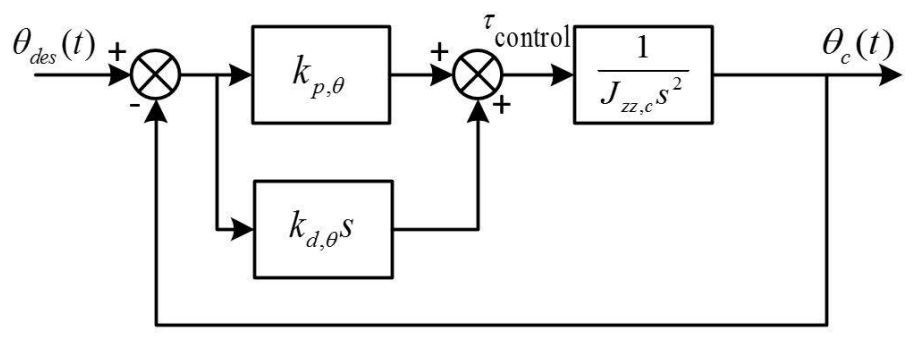

Figure 3.15: Block diagram of the $\mathrm{PD}$ controller for rotational control, where $\theta_{\text {des }}(t)$ is the desired angle and $\theta_{c}(t)$ is the actual angle.

of the chaser, $J_{z z, c}$. The proportional and derivative gains are denoted $k_{p, \theta}$ and $k_{d, \theta}$, and the desired and actual angles are $\theta_{\text {des }}(t)$ and $\theta_{c}(t)$, respectively. The closed-loop transfer function for this system is:

$$
\frac{\theta_{c}(s)}{\theta_{d e s}(s)}=\frac{\frac{k_{p, \theta}}{J_{z z, c}}}{s^{2}+\frac{k_{d, \theta}}{J_{z z, c}} s+\frac{k_{p, \theta}}{J_{z z, c}}}
$$


Comparing terms with Eq. (3.6), the control gain equations are found to be

$$
\begin{aligned}
& k_{p, \theta}=\omega_{n}^{2} J_{z z, c} \\
& k_{d, \theta}=2 \zeta \omega_{n} J_{z z, c}
\end{aligned}
$$

where the same $\zeta$ and $\omega_{n}$ as the translational controller are used. The control torque is calculated as follows

$$
\tau_{\text {control }}=k_{p, \theta} \theta_{e}+k_{d, \theta} \dot{\theta}_{e}
$$

where $\theta_{e}$ is the angle error, defined as

$$
\theta_{e}=\theta_{c}(t)-\theta_{\text {des }}(t)
$$

The presented controllers are used whenever position or attitude control is required of the spacecraft platforms. The discussion of the experimental facility provided in this chapter gives context to the planar equations of motion for tethered spacecraft that will be presented and numerically simulated in the following chapter. 


\section{Chapter 4}

\section{Planar Detumbling}

This chapter investigates the planar dynamics of a tethered spacecraft system. The reason for redefining the dynamics in a planar environment is to allow them to be validated through the planar experimental facility presented in Chapter 3. The presently accepted single tether and novel sub-tether configurations are presented. Simulations comparing the ability of the two tether configurations to stabilize a spinning debris are shown. Through numerical simulation, it was shown that the proposed sub-tether configuration significantly outperforms the single tether configuration at stabilizing an uncooperative debris.

\subsection{Introduction}

This chapter derives the equations of motion for a tethered spacecraft system in a planar environment. Effectively, this chapter presents a stripped down version of the three-dimensional dynamics presented in Chapter 2. After the dynamics are presented, numerical simulations are performed to compare the two tether configurations. Two simulations are shown, namely:

1. A chaser captures a spinning debris, and uses its thrusters to accelerate away from the debris. This causes tension in the tethers required to stabilize the debris.

2. A spinning debris is captured while the chaser and debris spin around each other. This creates a centripetal force in the tether that stabilizes the spinning debris. 


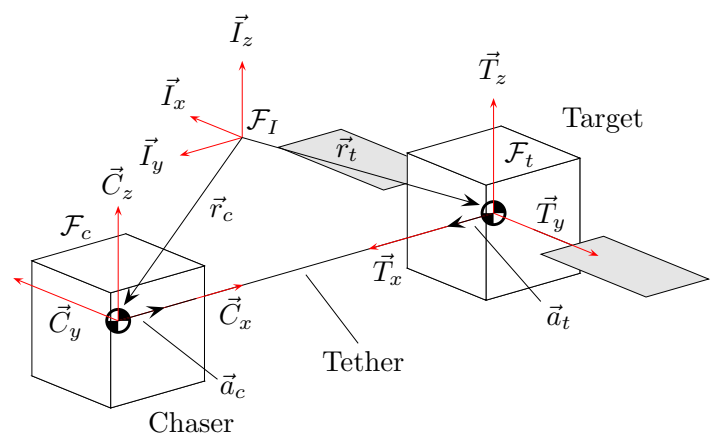

(a) Single tether configuration with reference frame (b) Sub-tether configuration. For clarity, $\mathcal{F}_{c}, \mathcal{F}_{t}$, definition.

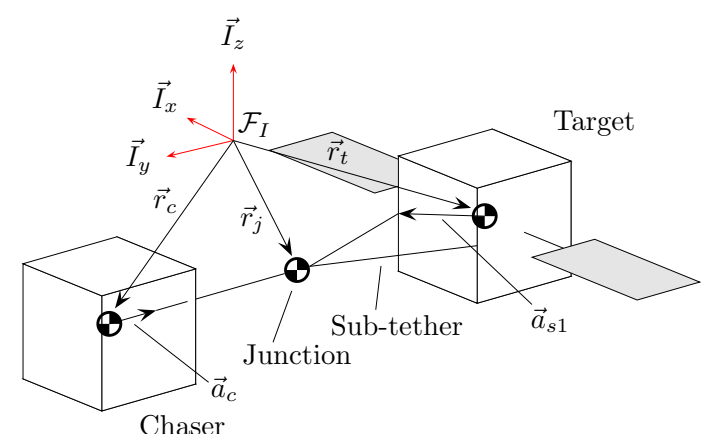
and $\vec{a}_{s 2}$ are omitted.

Figure 4.1: Reference frame and vector definition for planar dynamics modeling.

\subsection{Planar Dynamics}

Referring to Fig. 4.1, $\mathcal{F}_{I}$ represents the inertial reference frame, and vector $\vec{r}_{t}$ represents the position vector of the centre of mass of the target with respect to the origin of $\mathcal{F}_{I}$. The vector $\vec{r}_{t}$ has two-dimensional components, $\mathbf{r}_{t}$, in the inertial frame such that

$$
\vec{r}_{t}=\overrightarrow{\mathcal{F}}_{I}^{\mathrm{T}} \mathbf{r}_{t}
$$

Similarly, the two-dimensional positions of the centre of mass of the chaser and the junction are defined, respectively, as:

$$
\begin{aligned}
& \vec{r}_{c}=\overrightarrow{\mathcal{F}}_{I}^{\mathrm{T}} \mathbf{r}_{c} \\
& \vec{r}_{j}=\overrightarrow{\mathcal{F}}_{I}^{\mathrm{T}} \mathbf{r}_{j} .
\end{aligned}
$$

In the single tether configuration in Fig. 4.1(a), $\vec{a}_{t}$ denotes the two-dimensional attachment point vector of the single tether to the target, with respect to the target's centre of mass. Its components in the target body-fixed frame, $\mathcal{F}_{t}$, are given by:

$$
\mathbf{a}_{t} \equiv\left[\begin{array}{c}
a_{t, x} \\
a_{t, y}
\end{array}\right]=\overrightarrow{\mathcal{F}}_{t} \cdot \vec{a}_{t}
$$

where $\mathbf{a}_{t}$ represents the two-dimensional components of the vector $\vec{a}_{t}$ in $\mathcal{F}_{t}$. Similarly, the tether attachment point vector components in the chaser body-fixed reference 
frame, $\mathcal{F}_{c}$, are given by:

$$
\mathbf{a}_{c} \equiv\left[\begin{array}{l}
a_{c, x} \\
a_{c, y}
\end{array}\right]=\overrightarrow{\mathcal{F}}_{c} \cdot \vec{a}_{c}
$$

where $\vec{a}_{c}$ is the tether attachment point vector on the chaser with respect to the chaser's centre of mass. In the sub-tether configuration shown in Fig. 4.1(b), the two sub-tether attachment point vector components with respect to the target's centre of mass are

$$
\begin{aligned}
& \mathbf{a}_{s 1} \equiv\left[\begin{array}{l}
a_{s 1, x} \\
a_{s 1, y}
\end{array}\right]=\overrightarrow{\mathcal{F}}_{t} \cdot \vec{a}_{s 1} \\
& \mathbf{a}_{s 2} \equiv\left[\begin{array}{l}
a_{s 2, x} \\
a_{s 2, y}
\end{array}\right]=\overrightarrow{\mathcal{F}}_{t} \cdot \vec{a}_{s 2} .
\end{aligned}
$$

The angle $\theta_{t}$ represents the rotation about $\vec{I}_{z}$ to go from $\mathcal{F}_{I}$ to $\mathcal{F}_{t}$. Similarly, $\theta_{c}$ describes how $\mathcal{F}_{I}$ is rotated about $\vec{I}_{z}$ to obtain $\mathcal{F}_{c}$. The two-dimensional attitude matrix $\mathbf{A}(\theta)$ is a pure rotation of $\theta$ about the $\vec{I}_{z}$ axis which rotates vector components from a body frame to $\mathcal{F}_{I}$, given by

$$
\mathbf{A}(\theta)=\left[\begin{array}{rr}
\cos (\theta) & -\sin (\theta) \\
\sin (\theta) & \cos (\theta)
\end{array}\right]
$$

such that vector components are rotated from either body frame to $\mathcal{F}_{I}$, as follows:

$$
\mathbf{x}_{I}=\mathbf{A}(\theta) \mathbf{x}_{b}
$$

where $\mathbf{x}_{b}$ are the vector coordinates in a body frame and $\mathbf{x}_{I}$ are the vector components in $\mathcal{F}_{I}$.

With the above vector and reference frame definitions, the three degree-of-freedom (i.e., translation along $\vec{I}_{x}$ and $\vec{I}_{y}$ and rotation about $\vec{I}_{z}$ ) equations of motion for the system can now be presented. 


\subsubsection{Translational Motion}

The two degree-of-freedom translational equations of motion are governed by Newton's second Law:

$$
\mathbf{F}=m \ddot{\mathbf{r}}
$$

where $\mathbf{F}$ is the sum of the force vector components acting on any body in $\mathcal{F}_{I}, m$ is the mass of the spacecraft, and $\ddot{\mathbf{r}}$ is the resulting acceleration vector components in $\mathcal{F}_{I}$.

\subsubsection{Rotational Motion}

To model the single degree-of-freedom rotational dynamics, Euler's equations are used [8]

$$
\mathbf{J} \dot{\omega}+\omega^{\times}(\mathbf{J} \boldsymbol{\omega})=\tau
$$

where $\mathbf{J}$ is the inertia matrix of the spacecraft, $\boldsymbol{\omega}$ is the angular rate of the spacecraft, and $\boldsymbol{\tau}$ is the external torque applied to the body, all represented in the body-fixed reference frame. It is assumed that the body-fixed frame is aligned with the principal axes of the body, which yields an inertia matrix of

$$
\mathbf{J}=\left[\begin{array}{ccc}
J_{x x} & 0 & 0 \\
0 & J_{y y} & 0 \\
0 & 0 & J_{z z}
\end{array}\right]
$$

where $J_{i i} \forall i=\{x, y, z\}$ denotes the principal moments of inertia. Since the simulations and experiments performed are planar, the angular rate vector components will always have the form

$$
\boldsymbol{\omega}=\left[\begin{array}{l}
0 \\
0 \\
\omega
\end{array}\right]
$$

which eliminates the gyroscopic term in Eq. (4.11) and simplifies it to

$$
J_{z z} \dot{\omega}=\tau_{z} .
$$


In order to numerically simulate Eqs. (4.10) and (4.14), the forces and torques imparted on the chaser and the target must be quantified. Each tether is herein modeled as a nonlinear massless spring-damper system, as represented in Fig. 4.2. The following two sections present how the forces and torques are resolved for each

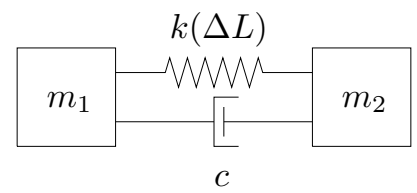

Figure 4.2: Simple spring-damper system, where two masses are attached via a spring and damper in parallel.

TSS configuration. Section 4.2.3 presents the equations for the single tether configuration shown in Fig. 4.1(a), and Sec. 4.2.4 presents the equations for the sub-tether configuration shown in Fig. 4.1(b).

\subsubsection{Single Tether Equations of Motion}

According to Fig. 4.1(a), the single tether TSS configuration has one tether that attaches the chaser to the target. Applying Eqs. (4.10) and (4.14), the equations of motion applied to this system are, for the passive target:

$$
\left[\begin{array}{c}
\ddot{r}_{t, x} \\
\ddot{r}_{t, y} \\
\dot{\omega}_{t}
\end{array}\right]=\left[\begin{array}{c}
\frac{-F_{s, x}}{m_{t}} \\
\frac{-F_{s, y}}{m_{t}} \\
\frac{\tau_{t}}{J_{z z, t}}
\end{array}\right]
$$

and for the active chaser:

$$
\left[\begin{array}{c}
\ddot{r}_{c, x} \\
\ddot{r}_{c, y} \\
\dot{\omega}_{c}
\end{array}\right]=\left[\begin{array}{c}
\frac{F_{s, x}+F_{c o n t r o l, x}}{m_{c}} \\
\frac{F_{s, y}+F_{c o n t r o l, y}}{m_{c}} \\
\frac{\tau_{c}+\tau_{c o n t r o l}}{J_{z z, c}}
\end{array}\right]
$$

where:

$$
\mathbf{F}_{s} \equiv\left[\begin{array}{c}
F_{s, x} \\
F_{s, y}
\end{array}\right], \quad \mathbf{F}_{\text {control }} \equiv\left[\begin{array}{c}
F_{\text {control }, x} \\
F_{\text {control }, y}
\end{array}\right]
$$


where $\mathbf{F}_{s}$ is the components of the force developed in the single tether in $\mathcal{F}_{I}$, and $\mathbf{F}_{\text {control }}$ is the components of the translational control thrust in $\mathcal{F}_{I}$. Components $\ddot{r}_{t, x}$ and $\ddot{r}_{t, y}$ are the two-dimensional acceleration components of $\ddot{\mathbf{r}}_{t}$, and $\ddot{r}_{c, x}$ and $\ddot{r}_{c, y}$ are the two-dimensional acceleration components of $\ddot{\mathbf{r}}_{c}$, all in $\mathcal{F}_{I}$. Variables $\dot{\omega}_{t}$ and $\dot{\omega}_{c}$ represent the angular acceleration of the target and chaser due to the torques imparted to them by the single tether, $\tau_{t}$ and $\tau_{c}$, in their respective body-fixed frames. Finally, $m_{t}, J_{z z, t}, m_{c}$, and $J_{z z, c}$ represent the mass and principal moment of inertia of the target and chaser, respectively. The remainder of this section will be used to determine the tether force $\mathbf{F}_{s}$, and resultant torques $\tau_{t}$ and $\tau_{c}$.

The current length of the single tether shown in Fig. 4.1(a) is found in $\mathcal{F}_{I}$ through

$$
\mathbf{L}_{s}=\mathbf{r}_{t}+\mathbf{A}\left(\theta_{t}\right) \mathbf{a}_{t}-\mathbf{r}_{c}-\mathbf{A}\left(\theta_{c}\right) \mathbf{a}_{c}
$$

where $\mathbf{L}_{s}$ is the components of the tether vector extending from the attachment point on the chaser to the attachment point on the target in $\mathcal{F}_{I}$, and all other variables have been previously defined. The stretch in the single tether is defined as:

$$
\Delta L_{s}=\left\|\mathbf{L}_{s}\right\|-L_{s, 0}
$$

where $L_{s, 0}$ is the unstretched length of the tether. The force developed by the single tether is the sum of the stiffness and viscous forces, calculated as

$$
\mathbf{F}_{s}=\left\{\begin{array}{rr}
{\left[k\left(\Delta L_{s}\right) \Delta L_{s}+c\left(\mathbf{v}_{t}+\mathbf{A}\left(\theta_{t}\right)\left[\begin{array}{r}
-\omega_{t} a_{t, y} \\
\omega_{t} a_{t, x}
\end{array}\right]-\mathbf{v}_{c}\right.\right.} & \\
\left.\left.-\mathbf{A}\left(\theta_{c}\right)\left[\begin{array}{r}
-\omega_{c} a_{c, y} \\
\omega_{c} a_{c, x}
\end{array}\right]\right)^{\mathrm{T}} \frac{\mathbf{L}_{s}}{\left\|\mathbf{L}_{s}\right\|}\right] \frac{\mathbf{L}_{s}}{\left\|\mathbf{L}_{s}\right\|}, & \text { for } \Delta L_{s}>0 \\
0, & \text { otherwise }
\end{array}\right.
$$

where $k\left(\Delta L_{s}\right)$ is the nonlinear stiffness of the tether material (as a function of the stretch, $\left.\Delta L_{s}\right), c$ is the damping coefficient of the tether, $\mathbf{v}_{t}$ and $\mathbf{v}_{c}$ are the velocities of the target and chaser in $\mathcal{F}_{I}$, respectively, and $\omega_{t}$ and $\omega_{c}$ are the angular rates of 
the target and chaser, in their respective body frames. It should be noted that the generated force, $\mathbf{F}_{s}$, is non-zero only when the tether is stretched.

The calculation to determine the torque that the single tether delivers to each spacecraft is performed in $\mathcal{F}_{t}$ and $\mathcal{F}_{c}$, as shown in Fig. 4.1(a). Therefore, the tether force vector components found in Eq. (4.20) must be rotated into each body frame using the attitude matrix, i.e.,

$$
\begin{aligned}
\mathbf{F}_{s}^{t} & \equiv\left[\begin{array}{c}
F_{s, x}^{t} \\
F_{s, y}^{t}
\end{array}\right]=\mathbf{A}\left(\theta_{t}\right)^{\mathrm{T}} \mathbf{F}_{s} \\
\mathbf{F}_{s}^{c} & \equiv\left[\begin{array}{c}
F_{s, x}^{c} \\
F_{s, y}^{c}
\end{array}\right]=\mathbf{A}\left(\theta_{c}\right)^{\mathrm{T}} \mathbf{F}_{s}
\end{aligned}
$$

where $\mathbf{F}_{s}^{t}$ is the single tether force expressed in $\mathcal{F}_{t}$ and $\mathbf{F}_{s}^{c}$ is the same in $\mathcal{F}_{c}$. The torque that the single tether delivers to each spacecraft is found via

$$
\begin{aligned}
& \tau_{t}=a_{t, y} F_{s, x}^{t}-a_{t, x} F_{s, y}^{t} \\
& \tau_{c}=a_{c, x} F_{s, y}^{c}-a_{c, y} F_{s, x}^{c} .
\end{aligned}
$$

In order to numerically simulate the motion, the forces and torques generated by the tether and control actuators are used with Eqs. (4.15) and (4.16). The following section presents the sub-tether TSS configuration equations of motion.

\subsubsection{Sub-Tether Equations of Motion}

The sub-tether configuration has a main tether that attaches to the chaser and branches into two sub-tethers that are attached to the target at various locations, as shown in Fig. 4.1(b). The point where the main tether branches into two sub-tethers is denoted the junction and is treated as a small point mass. Applying Eqs. (4.10) and (4.14) to this system, the equations of motion are, for the target:

$$
\left[\begin{array}{c}
\ddot{r}_{t, x} \\
\ddot{r}_{t, y} \\
\dot{\omega}_{t}
\end{array}\right]=\left[\begin{array}{c}
\frac{-\left(F_{s 1, x}+F_{s 2, x}\right)}{m_{t}} \\
\frac{-\left(F_{s 1, y}+F_{s 2, y}\right)}{m_{t}} \\
\frac{\tau_{t, s}+\tau_{t, s 2}}{J_{z z, t}}
\end{array}\right]
$$


for the junction:

$$
\left[\begin{array}{c}
\ddot{r}_{j, x} \\
\ddot{r}_{j, y}
\end{array}\right]=\left[\begin{array}{c}
\frac{F_{s 1, x}+F_{s 2, x}-F_{m, x}}{m_{j}} \\
\frac{F_{s 1, y}+F_{s 2, y}-F_{m, y}}{m_{j}}
\end{array}\right]
$$

and for the chaser:

$$
\left[\begin{array}{c}
\ddot{r}_{c, x} \\
\ddot{r}_{c, y} \\
\dot{\omega}_{c}
\end{array}\right]=\left[\begin{array}{c}
\frac{F_{m, x}+F_{\text {control }, x}}{m_{c}} \\
\frac{F_{m, y}+F_{c o n t r o l, y}}{m_{c}} \\
\frac{\tau_{c, m}+\tau_{c o n t r o l}}{J_{z z, c}}
\end{array}\right]
$$

where:

$$
\mathbf{F}_{m} \equiv\left[\begin{array}{c}
F_{m, x} \\
F_{m, y}
\end{array}\right], \quad \mathbf{F}_{s 1} \equiv\left[\begin{array}{c}
F_{s 1, x} \\
F_{s 1, y}
\end{array}\right], \quad \mathbf{F}_{s 2} \equiv\left[\begin{array}{c}
F_{s 2, x} \\
F_{s 2, y}
\end{array}\right]
$$

where $\mathbf{F}_{m}$ represents the force vector components of the main tether in $\mathcal{F}_{I}, \mathbf{F}_{s 1}$ and $\mathbf{F}_{s 2}$ represent the force vector components of the two sub-tethers in $\mathcal{F}_{I}, \tau_{t, s 1}$ and $\tau_{t, s 2}$ denote the torques imparted to the target due to the two sub-tethers in $\mathcal{F}_{t}$, and $\tau_{c, m}$ denotes the torque applied to the chaser from the main tether segment in $\mathcal{F}_{c}$. The remainder of this section will present the derivation of $\mathbf{F}_{m}, \mathbf{F}_{s 1}, \mathbf{F}_{s 2}, \tau_{c, m}, \tau_{t, s 1}$, and $\tau_{t, s 2}$.

The main tether vector, $\vec{L}_{m}$, attaches the chaser to the junction. The two subtether vectors, $\vec{L}_{s 1}$ and $\vec{L}_{s 2}$, extend from the junction to the two attachment points on the target. The tether vector coordinates in $\mathcal{F}_{I}$ are given by

$$
\begin{aligned}
\mathbf{L}_{m} & =\mathbf{r}_{j}-\mathbf{r}_{c}-\mathbf{A}\left(\theta_{c}\right) \mathbf{a}_{c} \\
\mathbf{L}_{s 1} & =\mathbf{r}_{t}+\mathbf{A}\left(\theta_{t}\right) \mathbf{a}_{s 1}-\mathbf{r}_{j} \\
\mathbf{L}_{s 2} & =\mathbf{r}_{t}+\mathbf{A}\left(\theta_{t}\right) \mathbf{a}_{s 2}-\mathbf{r}_{j}
\end{aligned}
$$

where all variables have been previously defined. The stretches in the main tether, and the first and second sub-tethers are:

$$
\begin{aligned}
& \Delta L_{m}=\left\|\mathbf{L}_{m}\right\|-L_{m, 0} \\
& \Delta L_{s 1}=\left\|\mathbf{L}_{s 1}\right\|-L_{s 1,0} \\
& \Delta L_{s 2}=\left\|\mathbf{L}_{s 2}\right\|-L_{s 2,0}
\end{aligned}
$$


where $L_{m, 0}, L_{s 1,0}$, and $L_{s 2,0}$ are the unstretched lengths of the main tether and subtethers, respectively. The resulting forces in the main tether and sub-tethers are:

$\mathbf{F}_{m}=\left\{\begin{array}{lr}{\left[k\left(\Delta L_{m}\right) \Delta L_{m}+c\left(\mathbf{v}_{j}-\mathbf{v}_{c}-\mathbf{A}\left(\theta_{c}\right)\left[\begin{array}{c}-\omega_{c} a_{c, y} \\ \omega_{c} a_{c, x}\end{array}\right]\right)^{\mathrm{T}} \frac{\mathbf{L}_{m}}{\left\|\mathbf{L}_{m}\right\|}\right]} & \frac{\mathbf{L}_{m}}{\left\|\mathbf{L}_{m}\right\|}, \\ 0, & \text { for } \Delta L_{m}>0\end{array}\right.$

$\mathbf{F}_{s 1}=\left\{\begin{array}{lr}{\left[k\left(\Delta L_{s 1}\right) \Delta L_{s 1}+c\left(\mathbf{v}_{t}+\mathbf{A}\left(\theta_{t}\right)\left[\begin{array}{c}-\omega_{t} a_{s 1, y} \\ \omega_{t} a_{s 1, x}\end{array}\right]-\mathbf{v}_{j}\right)^{\mathrm{T}} \frac{\mathbf{L}_{s 1}}{\left\|\mathbf{L}_{s 1}\right\|}\right]} & \frac{\mathbf{L}_{s 1}}{\left\|\mathbf{L}_{s 1}\right\|}, \\ \mathbf{0} & \text { for } \Delta L_{s 1}>0\end{array}\right.$

$\mathbf{F}_{s 2}=\left\{\begin{array}{lr}{\left[k\left(\Delta L_{s 2}\right) \Delta L_{s 2}+c\left(\mathbf{v}_{t}+\mathbf{A}\left(\theta_{t}\right)\left[\begin{array}{r}-\omega_{t} a_{s 2, y} \\ \omega_{t} a_{s 2, x}\end{array}\right]-\mathbf{v}_{j}\right)^{\frac{\mathbf{L}_{s 2}}{\left\|\mathbf{L}_{s 2}\right\|}}\right] \frac{\mathbf{L}_{s 2}}{\left\|\mathbf{L}_{s 2}\right\|},} \\ 0 & \text { for } \Delta L_{s 2}>0 \\ \mathbf{0}, & \text { otherwise }\end{array}\right.$

where $\mathbf{v}_{j}$ is the velocity components of the junction in $\mathcal{F}_{I}$. The resulting forces, $\mathbf{F}_{m}, \mathbf{F}_{s 1}$, and $\mathbf{F}_{s 2}$ are calculated in $\mathcal{F}_{I}$, and are only non-zero when the tethers are stretched. The torque delivered to each spacecraft is expressed in its body frame. Therefore, as with the single tether configuration, the tether forces must be rotated 
into the appropriate body-fixed reference frame, through:

$$
\begin{aligned}
\mathbf{F}_{m}^{c} & \equiv\left[\begin{array}{c}
F_{m, x}^{c} \\
F_{m, y}^{c}
\end{array}\right]=\mathbf{A}\left(\theta_{c}\right)^{\mathrm{T}} \mathbf{F}_{m} \\
\mathbf{F}_{s 1}^{t} & \equiv\left[\begin{array}{c}
F_{s 1, x}^{t} \\
F_{s 1, y}^{t}
\end{array}\right]=\mathbf{A}\left(\theta_{t}\right)^{\mathrm{T}} \mathbf{F}_{s 1} \\
\mathbf{F}_{s 2}^{t} & \equiv\left[\begin{array}{c}
F_{s 2, x}^{t} \\
F_{s 2, y}^{t}
\end{array}\right]=\mathbf{A}\left(\theta_{t}\right)^{\mathrm{T}} \mathbf{F}_{s 2}
\end{aligned}
$$

where, $\mathbf{F}_{m}^{c}$ is the main tether force vector components in $\mathcal{F}_{c}$, and $\mathbf{F}_{s 1}^{t}$ and $\mathbf{F}_{s 2}^{t}$ are the force vector components of the two sub-tethers in $\mathcal{F}_{t}$. The resulting torques on the spacecraft are calculated as follows:

$$
\begin{aligned}
\tau_{t, s 1} & =a_{s 1, y} F_{s 1, x}^{t}-a_{s 1, x} F_{s 1, y}^{t} \\
\tau_{t, s 2} & =a_{s 2, y} F_{s 2, x}^{t}-a_{s 2, x} F_{s 2, y}^{t} \\
\tau_{c, m} & =a_{c, x} F_{m, y}^{c}-a_{c, y} F_{m, x}^{c}
\end{aligned}
$$

and are used with Eqs. (4.35)-(4.37) in Eqs. (4.25)-(4.27) to simulate the motion. Numerical simulations of the motion are presented in the following section.

\subsection{Numerical Simulations}

In order to maintain control of a target, tension in the tethers is required [46]. Two main techniques for generating tension in the tethers are herein considered. Those are:

1. Commanding the chaser to thrust away from the target, creating tension in the tethers that regulates the attitude of the target.

2. Spinning the entire TSS such that the centripetal force provides the tension required to stabilize the target.

Two numerical simulations are performed for each tether configuration, to demonstrate the effectiveness of these two modes of stabilization available for a TSS, and 
to compare the TSS configurations themselves. The parameters in Table 4.1 are held constant for each simulation. The proportional-derivative controller, given by

Table 4.1: Initial conditions and TSS parameters for planar simulations and experiments.

\begin{tabular}{cccccc}
\hline \hline Parameter & Value & Parameter & Value & \multicolumn{1}{c}{ Parameter } & Value \\
\hline$J_{z z, t}, \mathrm{~kg} \cdot \mathrm{m}^{2}$ & 0.22 & $L_{m, 0}, \mathrm{~m}$ & 0.28 & $c_{\text {single }}, \frac{\mathrm{N} \cdot \mathrm{s}}{\mathrm{m}}$ & 3.0 \\
$J_{z z, c}, \mathrm{~kg} \cdot \mathrm{m}^{2}$ & 0.30 & $L_{s 1,0}, \mathrm{~m}$ & 0.28 & $c_{s u b}, \frac{\mathrm{N} \cdot \mathrm{s}}{\mathrm{m}}$ & 0.9 \\
$m_{t}, \mathrm{~kg}$ & 12.19 & $L_{s 2,0}, \mathrm{~m}$ & 0.28 & $K_{P, \theta}$ & 0.33 \\
$m_{c}, \mathrm{~kg}$ & 17.24 & $L_{s, 0}, \mathrm{~m}$ & 0.54 & $K_{D, \theta}$ & 0.56 \\
$m_{j}, \mathrm{~kg}$ & 0.01 & $\mathbf{a}_{c}, \mathrm{~m}$ & {$[0.135 ;-0.009]$} & $\mathbf{K}_{P}$ & $19.1 \mathbf{I}_{2 \times 2}$ \\
$\theta_{c}, \mathrm{deg}$ & 180 & $\mathbf{a}_{s 1}, \mathrm{~m}$ & {$[0.110 ; 0.127]$} & $\mathbf{K}_{D}$ & $32.7 \mathbf{I}_{2 \times 2}$ \\
$\theta_{t}, \mathrm{deg}$ & 0 & $\mathbf{a}_{s 2}, \mathrm{~m}$ & {$[0.113 ;-0.094]$} & & \\
Time Step, $\mathrm{s}$ & 0.004 & $\mathbf{a}_{t}, \mathrm{~m}$ & {$[0.110 ; 0.016]$} & & \\
\hline \hline
\end{tabular}

Eqs. (3.10) and (3.15), is used for position and attitude control of the chaser platform, as previously explained in Sec. 3.3.2. The control actuation is governed by

$$
\mathbf{F}_{\text {control }}=\mathbf{K}_{P}\left[\mathbf{r}_{\text {des }}(t)-\mathbf{r}_{c}(t)\right]+\mathbf{K}_{D}\left[\dot{\mathbf{r}}_{\text {des }}(t)-\dot{\mathbf{r}}_{c}(t)\right]
$$

where $\mathbf{r}_{\text {des }}$ is the desired chaser position in $\mathcal{F}_{I}, \mathbf{K}_{P}$ is the proportional gain matrix and $\mathbf{K}_{D}$ is the derivative gain matrix, as listed in Table 4.1. The attitude controller is similarly obtained:

$$
\tau_{\text {control }}=K_{P, \theta}\left[\theta_{\text {des }}(t)-\theta(t)\right]+K_{D, \theta}\left[\dot{\theta}_{\text {des }}(t)-\dot{\theta}(t)\right]
$$

where $\theta_{\text {des }}(t)$ is the desired angle of the chaser as a function of time, and $K_{P, \theta}$ and $K_{D, \theta}$ represent the constant proportional and derivative control gains, respectively.

The details and results of each simulation case are presented in the following subsections. Results of the simulations include time histories of the target tether angle, the target angular rate, and the total angular momentum of the system. Figure 4.3 depicts the target tether angle, defined as a positive angle between $\vec{T}_{x}$ and the tether. It is calculated as

$$
\phi=\cos ^{-1}\left(\frac{-\left(\mathbf{A}(\theta) \mathbf{T}_{x}\right)^{\mathrm{T}} \mathbf{L}_{s}}{\left\|\mathbf{T}_{x}\right\|\left\|\mathbf{L}_{s}\right\|}\right)
$$




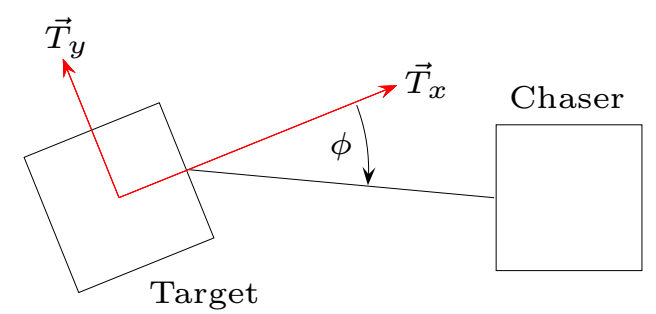

Figure 4.3: Positive angle $\phi$ represents the current angle between $\vec{T}_{x}$ and the tether. The sub-tether configuration uses an imaginary single tether to calculate the tether angle.

Table 4.2: Initial conditions for planar thrust stabilization simulations and experiments.

\begin{tabular}{cccc}
\hline \hline Parameter & Value & Parameter & Value \\
\hline $\mathbf{r}_{t}, \mathrm{~m}$ & {$[0.35 ; 1.19]$} & $\mathbf{v}_{t}, \mathrm{~m} / \mathrm{s}$ & {$[0 ; 0]$} \\
$\mathbf{r}_{c}, \mathrm{~m}$ & {$[1.10 ; 1.20]$} & $\mathbf{v}_{c}, \mathrm{~m} / \mathrm{s}$ & {$[0 ; 0]$} \\
$\omega_{t 0}, \operatorname{deg} / \mathrm{s}$ & 10 & Simulation Length, $\mathrm{s}$ & 100 \\
\hline \hline
\end{tabular}

where $\mathbf{T}_{x}$ is the vector components of the $\vec{T}_{x}$ axis expressed in $\mathcal{F}_{t}, \mathbf{A}(\theta)$ is the rotation matrix from $\mathcal{F}_{t}$ to $\mathcal{F}_{I}, \theta$ is the attitude of the target, and $\mathbf{L}_{s}$ is the single tether vector components in $\mathcal{F}_{I}$. A goal of the stabilization process is to regulate the tether angle, reduce the angular rates, and dissipate angular momentum from the system.

\subsubsection{Thrust Stabilization}

This section presents a technique for stabilizing a spinning target by continuously accelerating away from it. The acceleration maintains tension in the tethers which regulates the attitude of the target. The numerical simulation is performed in MATLAB/Simulink with initial conditions listed in Table 4.2. Both spacecraft are initially at rest and aligned such that all tethers are initially just tight. At time $t=2$, the target uses its thrusters to reach an angular rate of $\omega_{t 0}$. At the same time, the chaser initiates its control system to provide constant acceleration away from the target. To achieve the constant acceleration of the chaser, the desired position, $\mathbf{r}_{\text {des }}(t)$, is fed to the chaser's position control system and is varied through time to reach the position 


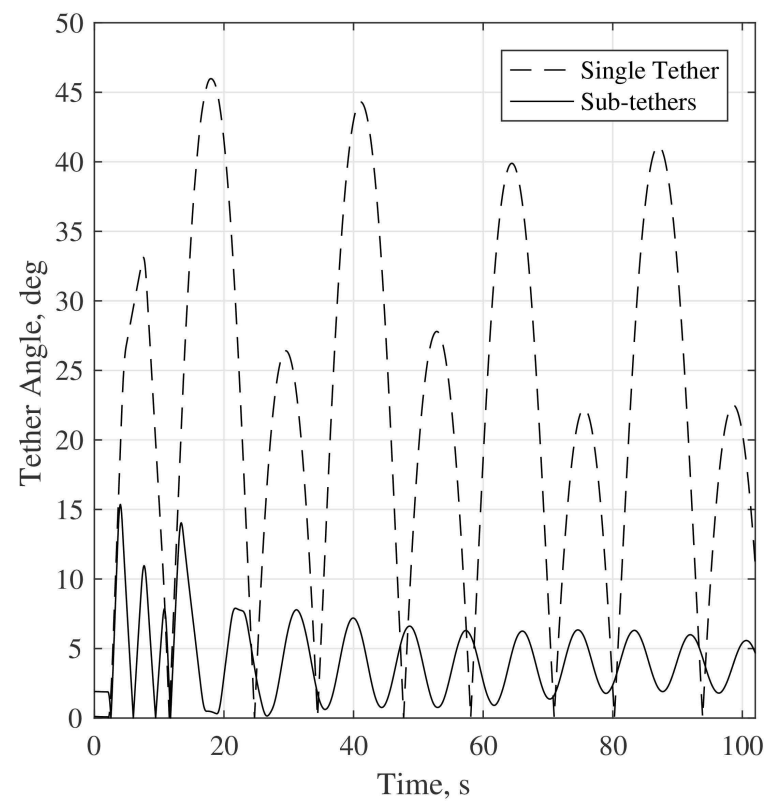

(a) Target tether angle

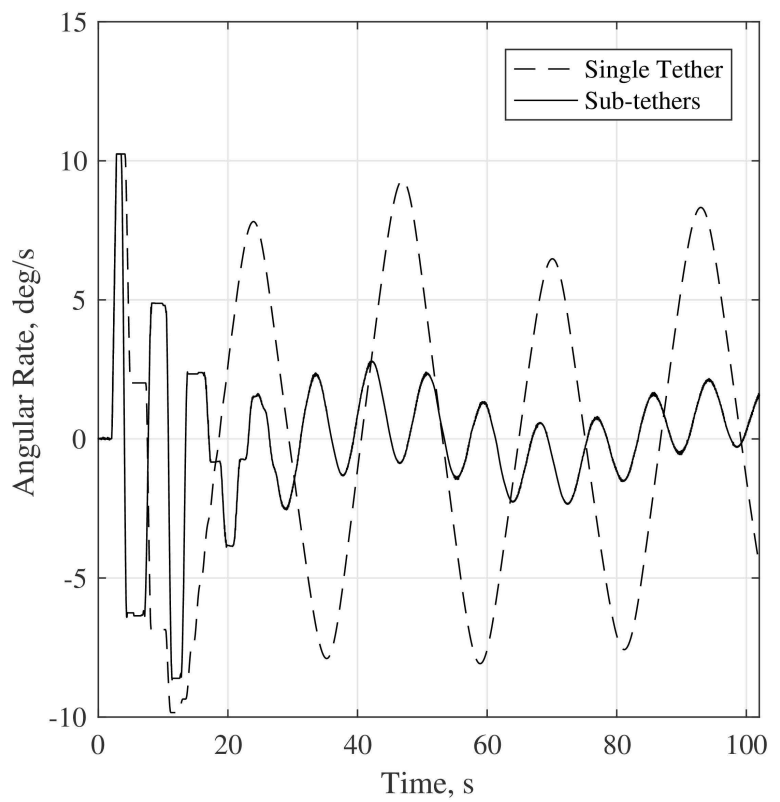

(b) Target angular rate

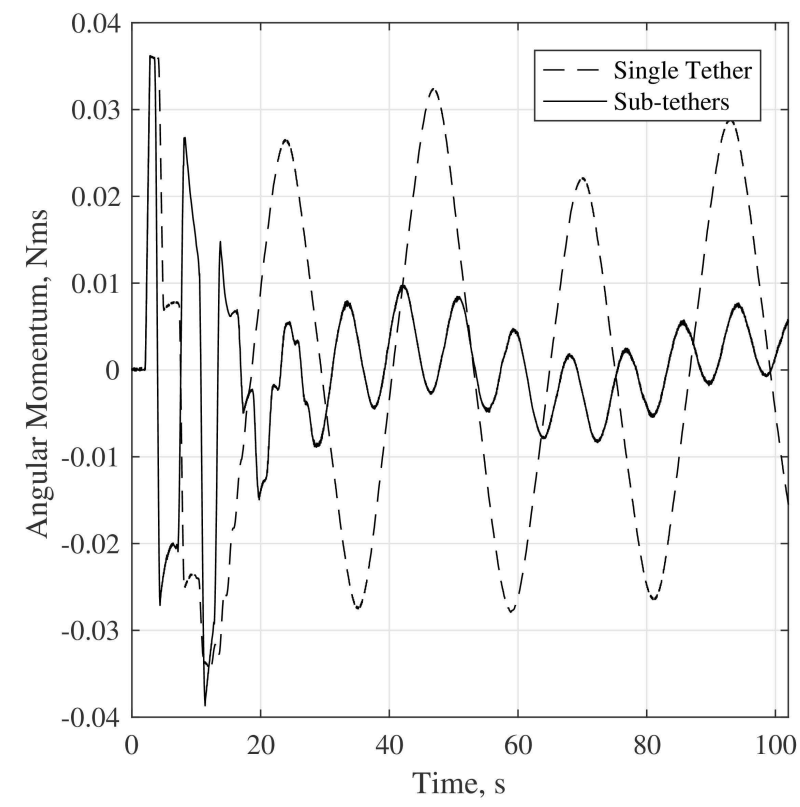

(c) Total angular momentum

Figure 4.4: Resulting simulated TSS motion for planar thrust stabilization, comparing the two tether configurations.

$\mathbf{r}_{f}=[3.10 ; 1.20] \mathrm{m}$ in $20 \mathrm{~s}$. Specifically, it is varied through

$$
\mathbf{r}_{\text {des }}(t)=\mathbf{r}_{0}+\mathbf{d} \frac{t^{2}}{2}
$$


where $\mathbf{d}$ is the desired constant acceleration, and $t$ is the time. The desired acceleration is calculated through

$$
\mathbf{d}=\frac{2\left(\mathbf{r}_{f}-\mathbf{r}_{0}\right)}{t^{2}}
$$

where $\mathbf{r}_{0}$ and $\mathbf{r}_{f}$ are the initial and final positions of the chaser in $\mathcal{F}_{I}$, and $t$ is the time taken to move from $\mathbf{r}_{0}$ to $\mathbf{r}_{f}$. The attitude control system on the chaser is set to continually hold its initial attitude at $\theta_{c}$. Both TSS configurations were simulated with identical initial conditions such that the two scenarios can readily be compared.

Figure 4.4 presents the results of the numerical simulation. Figure 4.4(a) shows the tether angle as a function of time. One of the objectives of the attitude stabilization strategy is to reduce this angle to zero. The proposed sub-tether configuration results in a tether angle that is considerably smaller than the single tether configuration for the same initial conditions.

Figure 4.4(b) shows the angular rate of the target as a function of time. Both TSS configurations regulate the angular rate of the target towards zero, but the sub-tether configuration does so significantly quicker. Stabilizing a target quickly after capture is highly desirable. This test scenario demonstrates how simply altering the tether configuration passively enhances the stabilization ability of the system. The proposed sub-tether configuration better exploits the damping properties of the tethers compared to the single tether configuration. Tether damping is the mechanism through which angular momentum is removed from the system. Figure 4.4(c) shows the total angular momentum of the system over time. The sub-tether configuration is more effective at dissipating angular momentum than the single tether configuration.

Figure 4.5 shows snapshots of an animation of the single tether motion, which can be found at https://goo.gl/lzgziR, and Fig. 4.6 shows snapshots of the sub-tether animation, which can be found at https://goo.gl/YgMvr2. The red box represents the chaser and the black box represents the target. The large black rectangle represents the size of the granite table that will be used for the experiments. Notice how at each frame of the animation, the sub-tether configuration leads the target to have a smaller tether angle than the single tether configuration.

Section 4.3.2 simulates another mode of maintaining tether tension to stabilize a spinning target. 
Time: $2 \mathrm{~s}$

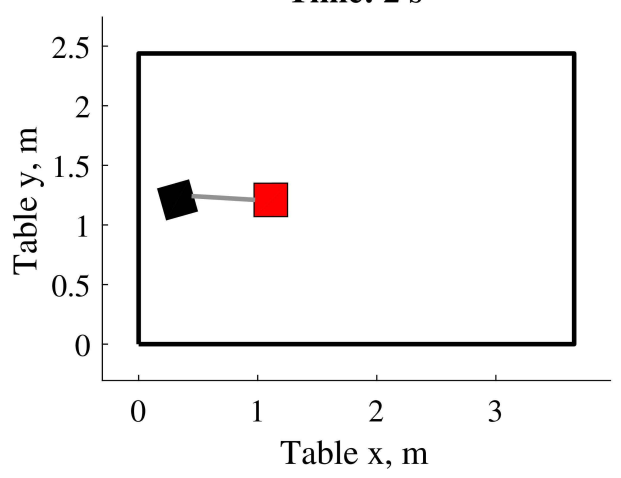

Time: $9 \mathrm{~s}$

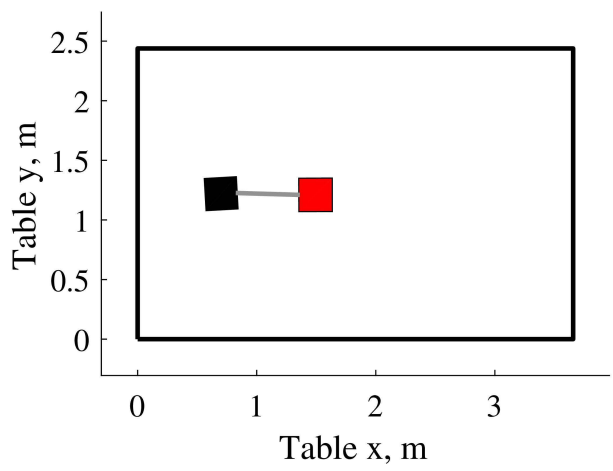

Time: $16 \mathrm{~s}$

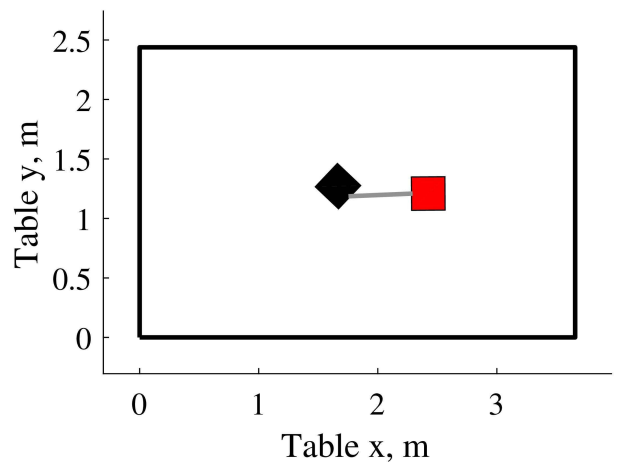

Time: $6 \mathrm{~s}$

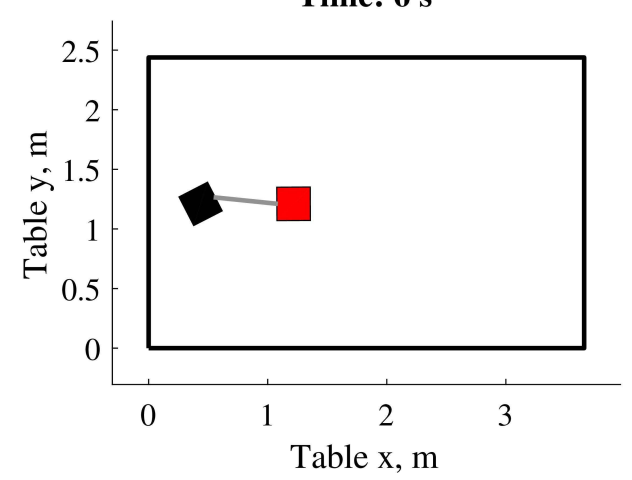

Time: $13 \mathrm{~s}$

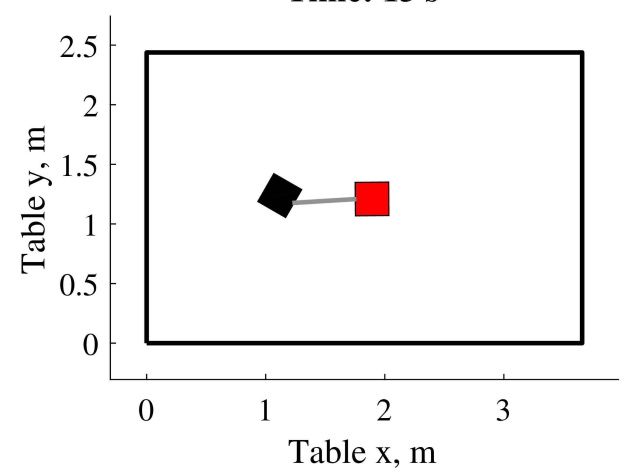

Time: $20 \mathrm{~s}$

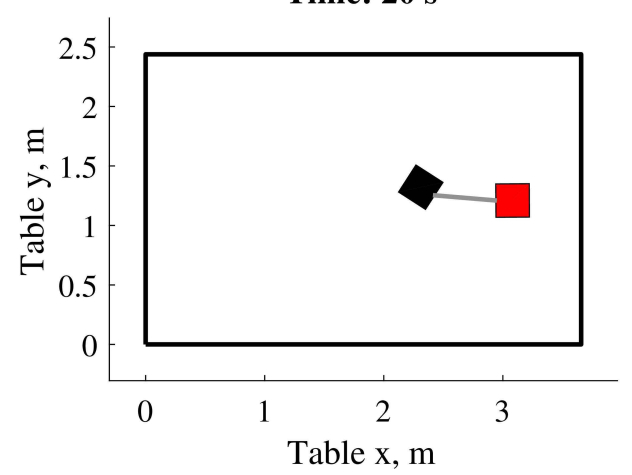

Figure 4.5: Snapshots of an animation of the resulting motion for the thrust stabilization simulation. The red spacecraft is the chaser and the black is the target. The single tether is shown in gray.

\subsubsection{TSS Spin Stabilization}

This section demonstrates another mode for stabilizing the target. That is, to initiate

a TSS spin such that the chaser and target are spinning about the barycentre of the TSS. The goal of this spin technique is to use the centripetal force generated 
Time: $2 \mathrm{~s}$

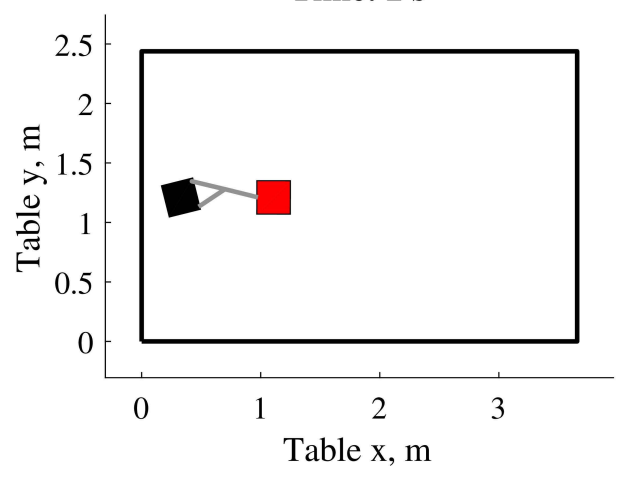

Time: $9 \mathrm{~s}$

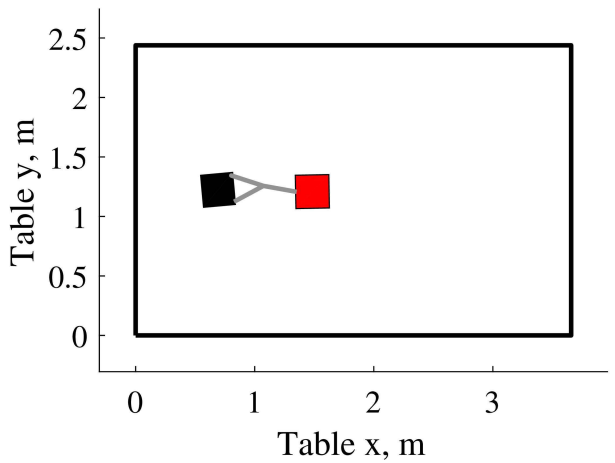

Time: $16 \mathrm{~s}$

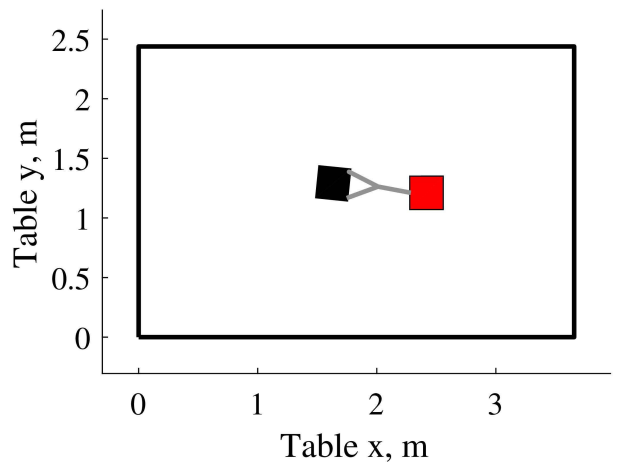

Time: $6 \mathrm{~s}$

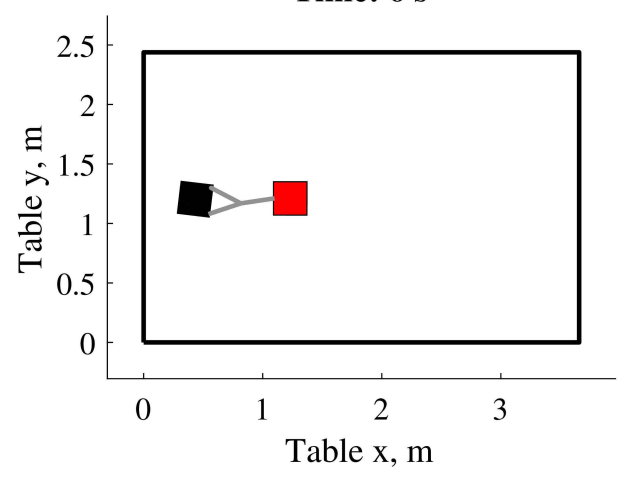

Time: $13 \mathrm{~s}$



Time: $20 \mathrm{~s}$

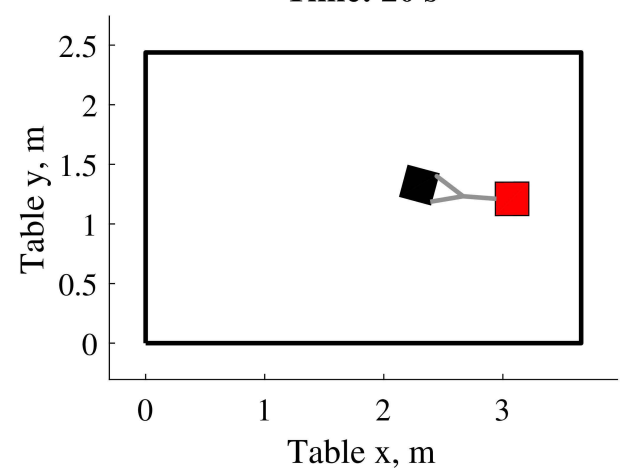

Figure 4.6: Snapshots of an animation of the resulting motion for the thrust stabilization simulation. The red spacecraft is the chaser and the black is the target. The sub-tethers are shown in gray. 
Table 4.3: Initial conditions for TSS spin planar simulations and experiments.

\begin{tabular}{cccc}
\hline \hline Parameter & Value & Parameter & Value \\
$\mathbf{r}_{t}, \mathrm{~m}$ & {$[2.02 ; 0.74]$} & $\mathbf{v}_{t}, \mathrm{~m} / \mathrm{s}$ & {$[0 ;-0.3]$} \\
$\mathbf{r}_{c}, \mathrm{~m}$ & {$[2.80 ; 0.75]$} & $\mathbf{v}_{c}, \mathrm{~m} / \mathrm{s}$ & {$[0 ; 0.2]$} \\
$\omega_{t 0}, \mathrm{deg} / \mathrm{s}$ & 79 & Simulation Length, $\mathrm{s}$ & 30 \\
\hline \hline
\end{tabular}

by the rotation to develop tension in the tethers and regulate the attitude of the uncooperative target. The main benefit of this technique is that once initiated, it does not consume fuel. Initial conditions for the simulation are shown in Table 4.3. The translational control system is not used on the chaser for this simulation. The spacecraft are initially positioned such that all tethers are barely under tension. The attitude control system is used to command the chaser to continually point its $\vec{C}_{x}$ axis toward the target. The desired chaser angle, $\theta_{\text {des }}$, used in the control system is calculated as follows

$$
\theta_{d e s}=\tan ^{-1}\left(\frac{r_{t, y}-r_{c, y}}{r_{t, x}-r_{c, x}}\right)
$$

where $r_{t, y}$ and $r_{c, y}$ are the $\vec{I}_{y}$ components of $\mathbf{r}_{t}$ and $\mathbf{r}_{c}$, respectively, and $r_{t, x}$ and $r_{c, x}$ are the $\vec{I}_{x}$ components of $\mathbf{r}_{t}$ and $\mathbf{r}_{c}$. The target is given an angular rate of $\omega_{t 0}$ at $t=2 \mathrm{~s}$. Plots of the resulting motion are shown in Fig. 4.7.

Simulation results show how spinning the TSS is a valid technique for stabilizing an uncooperative spacecraft, and how the proposed sub-tether configuration is more efficient than the single tether configuration. Figures 4.7(a) and 4.7(b) show that while the single tether is reducing the tether angle and angular rate of the target, it does so much slower than the sub-tether configuration. The angular rate in Fig. 4.7(b) does not tend toward zero but converges to the spin rate of the TSS. A similar trend is observed in the total angular momentum plot in Fig. 4.7(c).

Snapshots of the simulated motion are shown in Fig. 4.8 for the single tether configuration, and an animation can be found at https://goo.gl/8y8H31. Figure 4.9 shows the corresponding snapshots for the sub-tether configuration, with an animation available at https://goo.gl/tOsIzu. Notice how over time, the sub-tether configuration keeps the tether angle very low. 


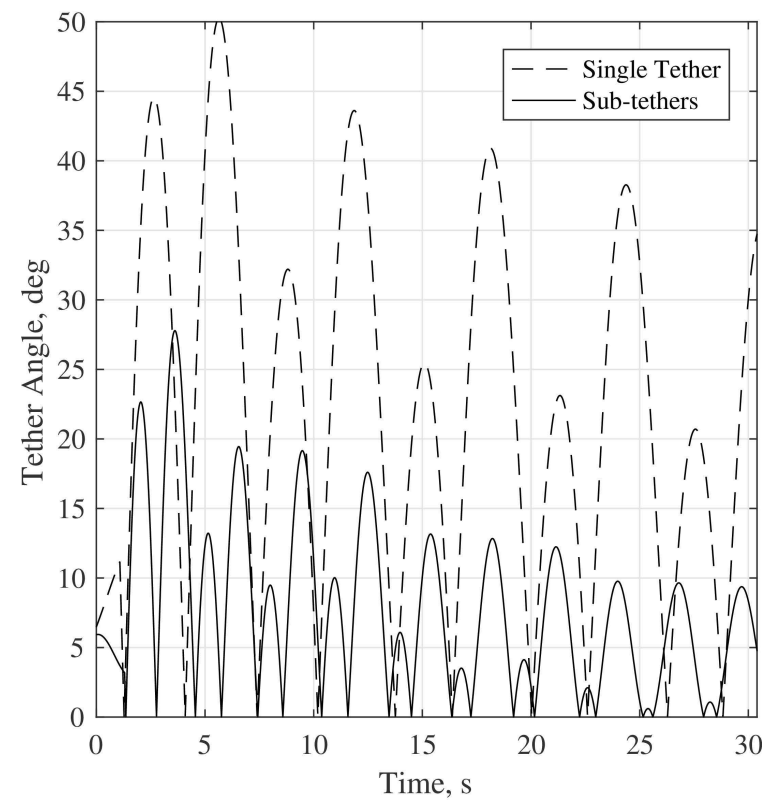

(a) Target tether angle

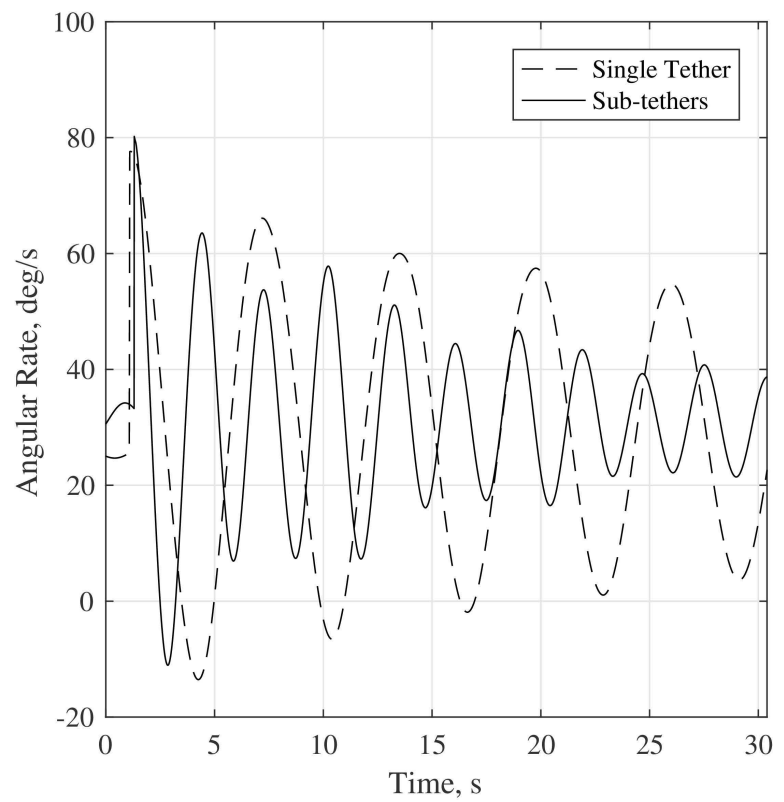

(b) Target angular rate

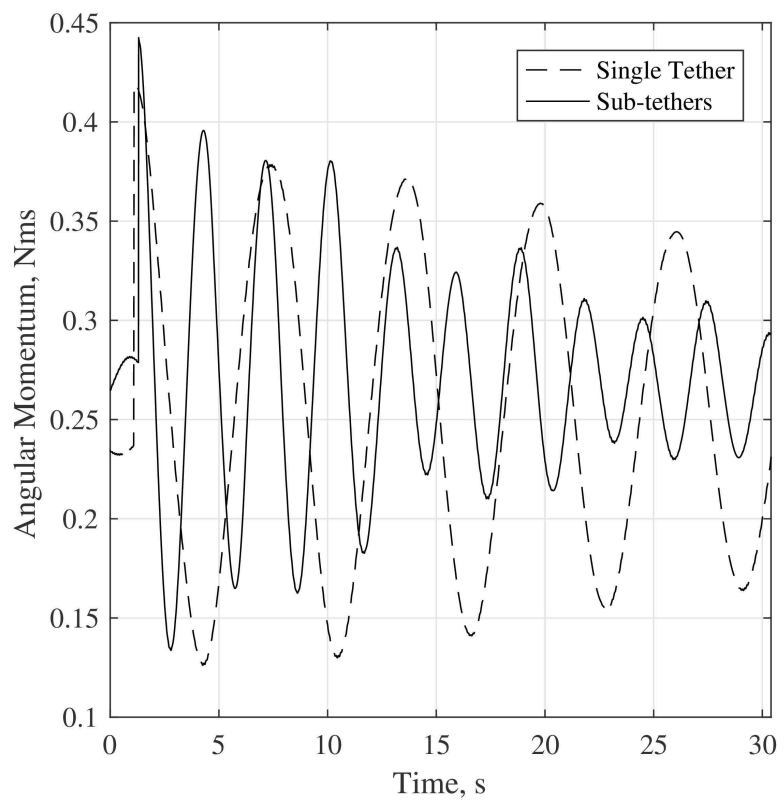

(c) Total angular momentum

Figure 4.7: Resulting simulated motion for TSS spin stabilization, comparing the two tether configurations.

To validate the numerical simulations, experiments are performed. The following chapter describes the experiments that were performed, and presents and discusses experimental results. 
Time: $2 \mathrm{~s}$

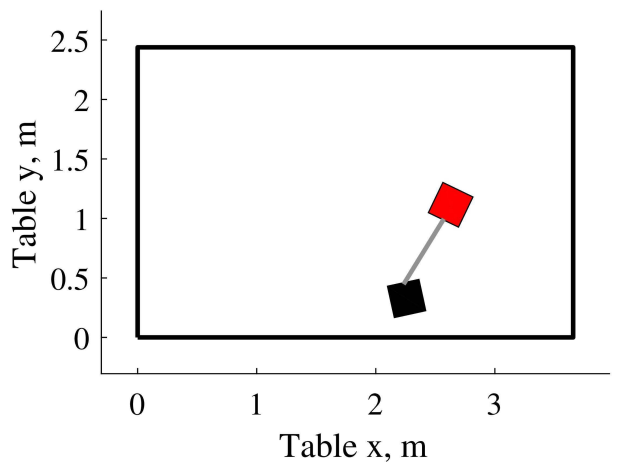

Time: $17 \mathrm{~s}$

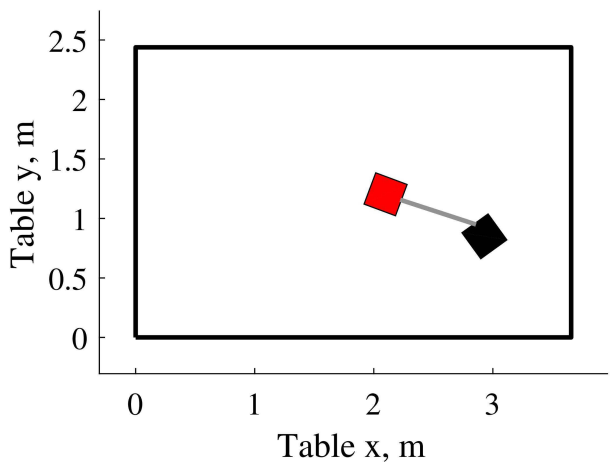

Time: $32 \mathrm{~s}$

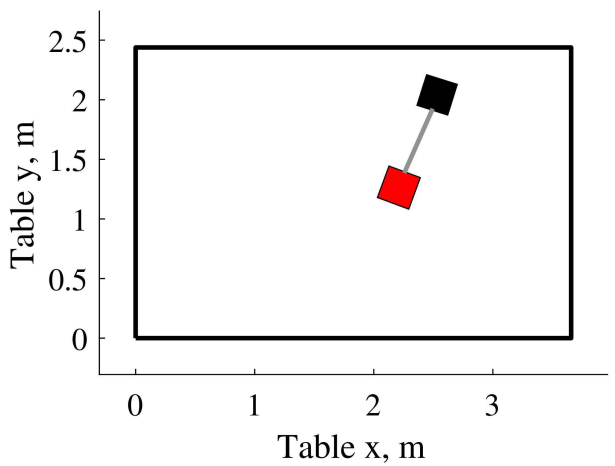

Time: $9 \mathrm{~s}$



Time: $25 \mathrm{~s}$

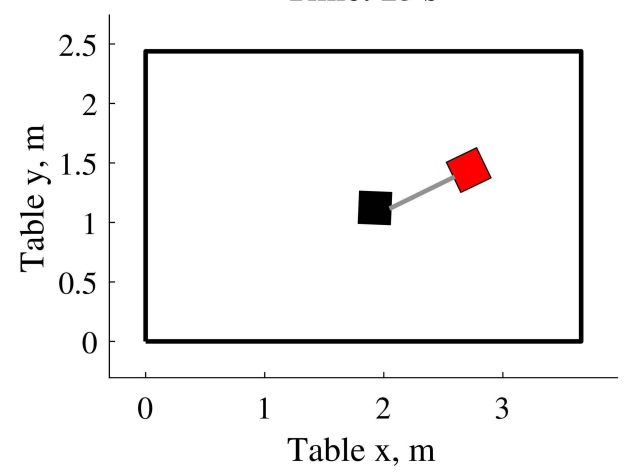

Time: $40 \mathrm{~s}$

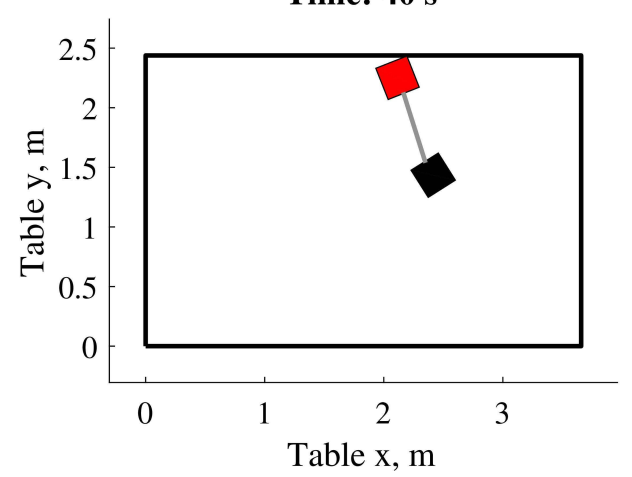

Figure 4.8: Snapshots of an animation of the resulting motion for the TSS spin stabilization simulation. The red spacecraft is the chaser and the black is the target. The single tether is shown in gray. 
Time: $2 \mathrm{~s}$

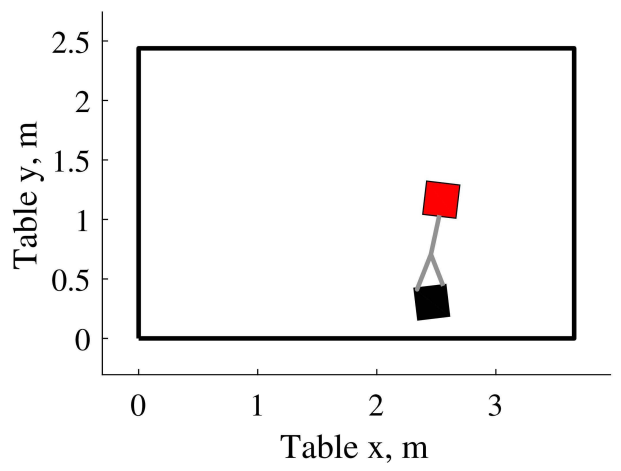

Time: $17 \mathrm{~s}$

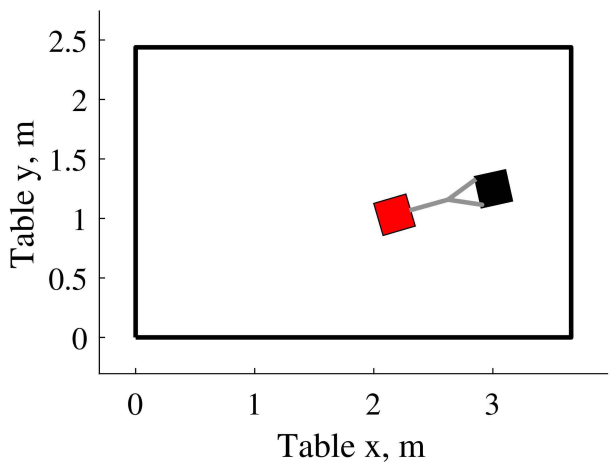

Time: $32 \mathrm{~s}$

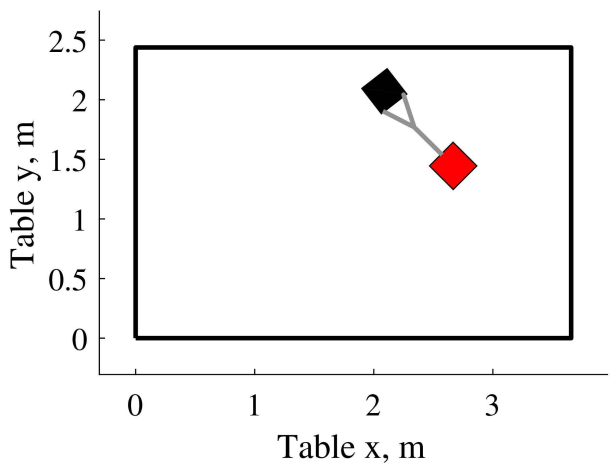

Time: $9 \mathrm{~s}$

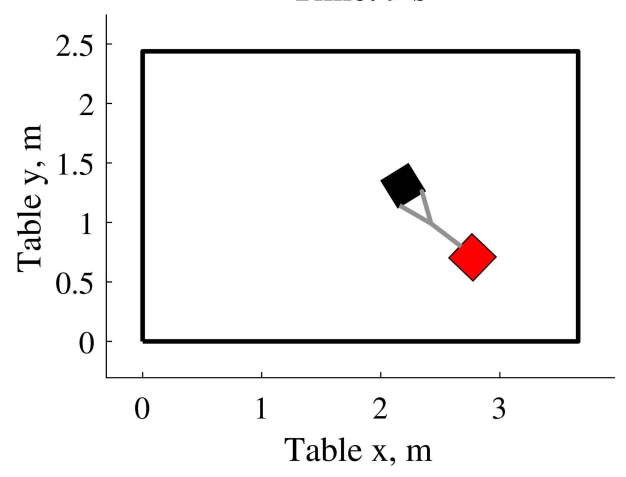

Time: $25 \mathrm{~s}$

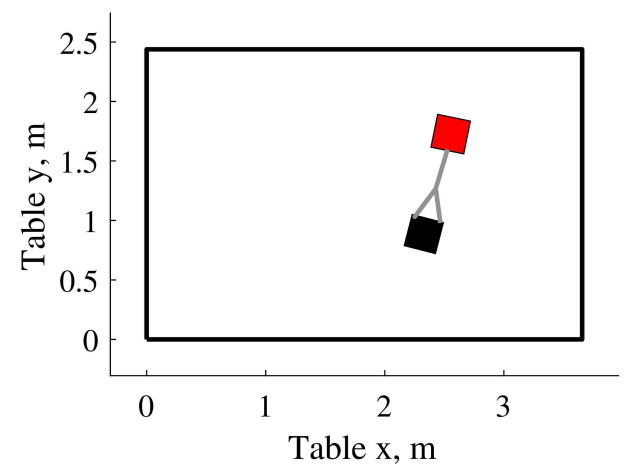

Time: $40 \mathrm{~s}$

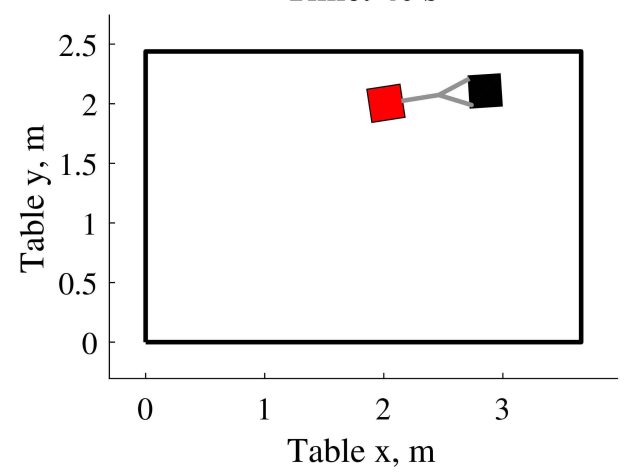

Figure 4.9: Snapshots of an animation of the resulting motion for the TSS spin stabilization simulation. The red spacecraft is the chaser and the black is the target. The sub-tethers are shown in gray. 


\section{Chapter 5}

\section{Experimental Validation}

Experiments are performed in the Spacecraft Robotics and Control Laboratory at Carleton University to test the effect of tether configuration on the ability to stabilize a tumbling target. The spin scenario shown in simulation was replicated in experiment. Experimental data shows excellent correlation with simulated results. The numerical simulations were successfully validated. The experiments performed are, to the best of the author's knowledge, the first experiments to date concerning the detumbling of a spinning debris using tethers.

\subsection{Introduction}

A planar gravity-offset testbed is used, where two spacecraft platforms are tethered together on a flat granite surface, as discussed in Chap. 3. The two TSS configurations numerically simulated in Chap. 4 are replicated in experiments.

The simulation cases presented in Sec. 4.3 were chosen such that they are replicable experimentally. Experiment properties are the same as in Table 4.1.

The experiment design consists of two spacecraft platforms placed on the flat table and tethered together using the tethers that were modeled in simulations. Figure 5.1 shows the two SPOT spacecraft tethered together on the flat table in the sub-tether configuration.

The platforms sit on the table until a strong lock has been acquired on the LEDs by the ground truth system. Following this, the platforms begin to float and spend 60 seconds moving to the desired initial position on the table and come to rest.

To establish the spinning and tumbling initial conditions for the TSS spin technique discussed in Sec. 4.3.2, both spacecraft exert equal thrust perpendicular to the line joining the two platforms, in opposite directions, for 10 seconds. This establishes a TSS spin about the barycentre of the system. Once the spin has been initiated, the 


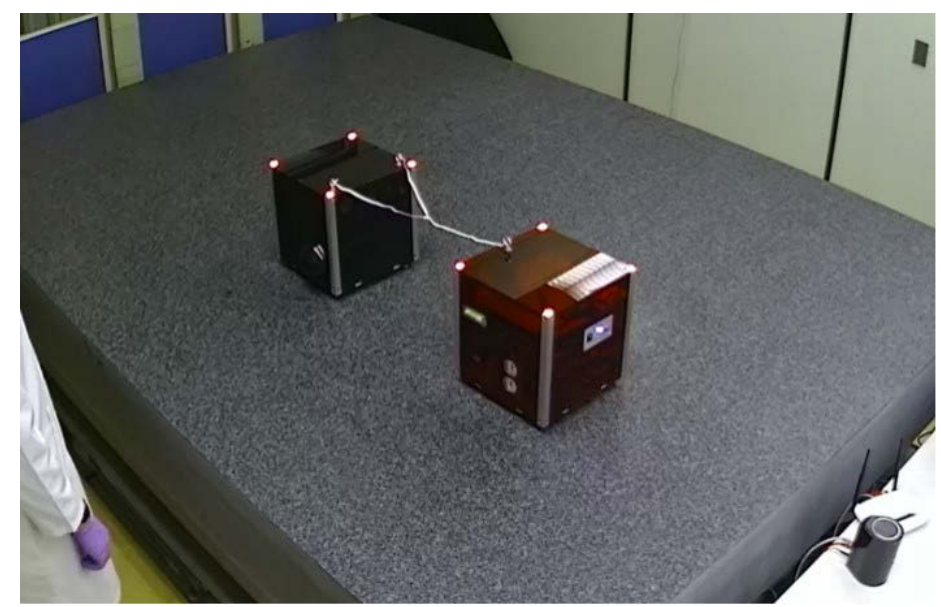

Figure 5.1: Two spacecraft platforms tethered together on the planar gravity-offset testbed at Carleton's SPOT.

operator manually provides a disturbance to the target to initiate a tumbling motion. This creates the initial conditions listed in Table 4.3. Once the tumbling has been initiated, the target becomes fully passive, and the chaser uses its thrusters solely to maintain its $\vec{C}_{x}$ axis pointing at the target. The system coasts for 35 seconds, drifting due to the slight slope in the table, while the tethers and sub-tethers reduce the tether angle of the target and dissipate some of the angular momentum in the system.

\subsection{Single Tether Results}

The experiment was first performed with the platforms in the single tether configuration. A video of the single tether experiment can be found at: https://goo. gl/xoXGIB. Results from the single tether TSS spin experiments are compared to simulated results in Fig. 5.2. These data constitute some of the first published results from the SPOT facility.

The tether angle described in Fig. 4.3 is shown in Fig. 5.2(a). The similarities between the simulated and experimental results are obvious, with only small discrepancies in the amplitude of the oscillations. These discrepancies may be due to numerous sources, including: uncertainties in mass properties of the chaser or target, an inaccurate tether model, unmodeled effects such as drag or friction between the 


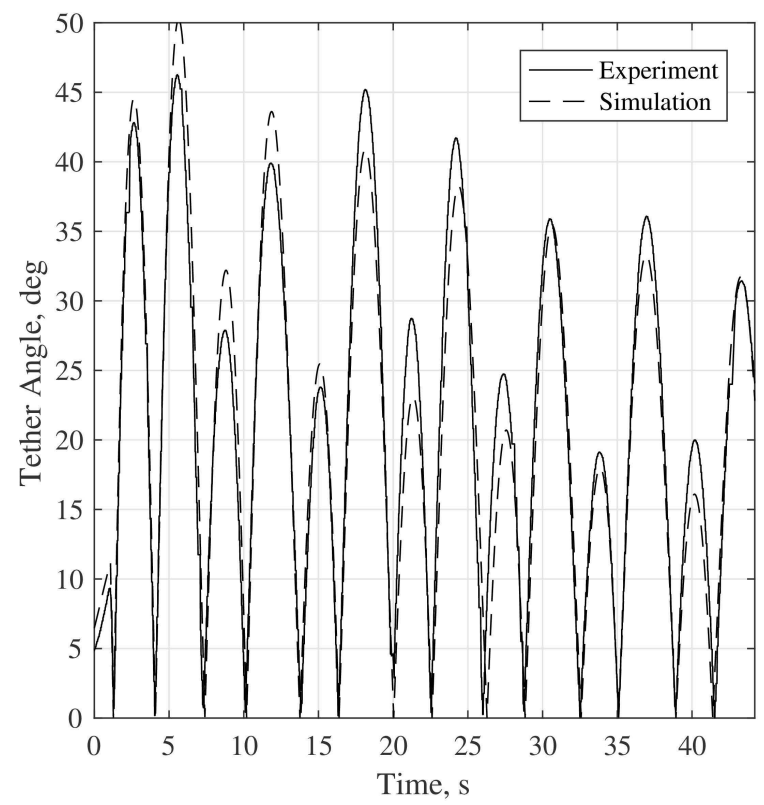

(a) Target tether angle

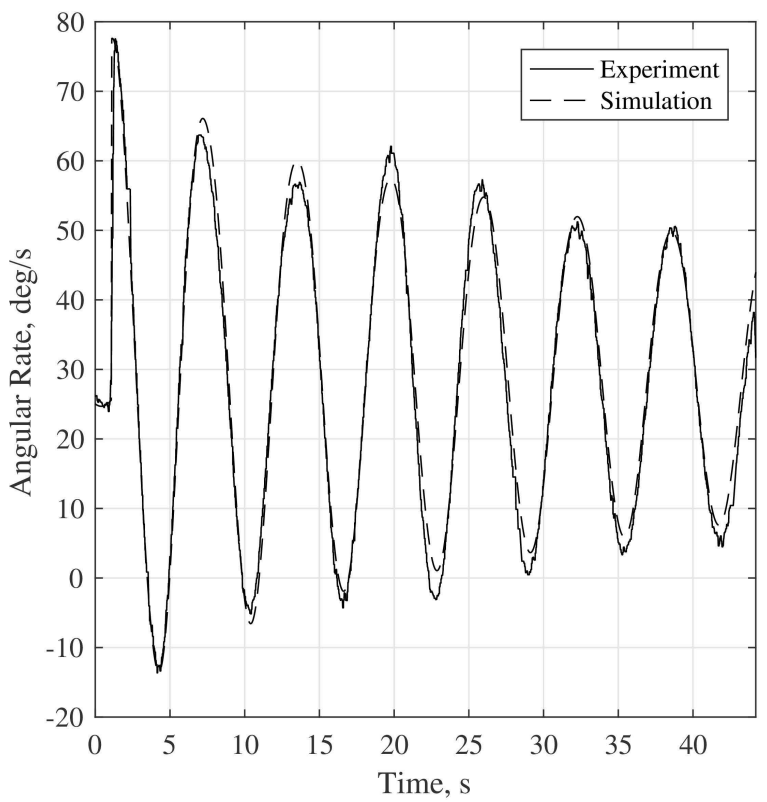

(b) Target angular rate

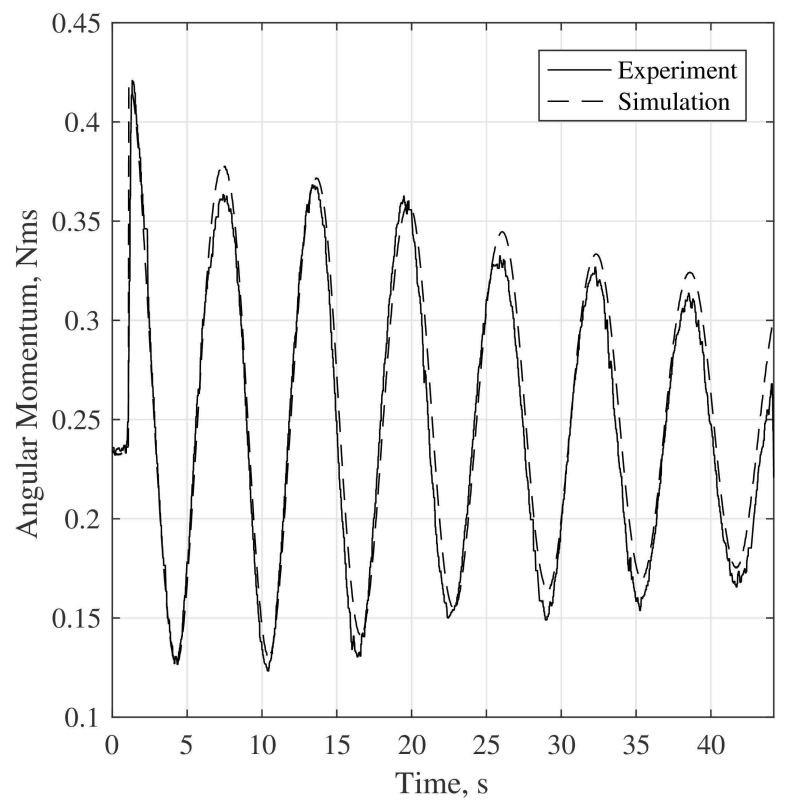

(c) Total angular momentum

Figure 5.2: Comparing experimental and simulated response for the planar TSS spin stabilization technique with a single tether.

spacecraft and the flat table, or signal noise. The plot of the angular rate over time, shown in Fig. 5.2(b), also has excellent correlation. The angular momentum of the entire system is calculated and shown in Fig. 5.2(c), showing that the initial angular 
momentum due to the tumbling of the target slowly dissipates such that the total angular momentum tends towards the steady state angular momentum of the TSS spinning about its barycentre. The angular momentum in the experiment tends toward a lower final angular momentum quantity than in simulation, and is attributed to the unmodeled air drag and residual friction slowing down the two spacecraft from spinning about the barycentre of the TSS. The experimental results successfully validate the presented single tether model.

\subsection{Sub-tether Results}

The experiment was repeated with the sub-tether configuration. A video of the subtether experiment can be found at https://goo.gl/PXBtju. Experimental results are compared to simulated results in Fig. 5.3.

The experiment and simulation are well correlated once again. Figure 5.3(a) shows minor tether angle discrepancies in amplitude but a nearly identical trend. The oscillations quickly decay to around 5 deg in 30 seconds, indicating the target tumbling has been quickly regulated. The target angular rate shown in Fig. 5.3(b) show a quick decay that converges to the TSS spin rate. In fact, the steady state angular rate is slightly lower in experiment as compared to simulation, which is again likely due to drag and friction slowing the TSS spin rate over time. The tethers effectively dissipate the angular momentum in the system, as demonstrated in Fig. 5.3(c). The sub-tether damping quickly dissipates the angular momentum in the system that is a result of the tumbling motion of the target. The correlation between the experimental and simulated results demonstrate the validity of the presented equations of motion developed in Chap. 4.

The single tether and sub-tether experimental responses are compared in Fig. 5.4. All three plots show that the sub-tether configuration significantly outperforms the single tether configuration when it comes to detumbling a passive target, all else being equal for all practical purposes. Both the tether angle, shown in Fig. 5.4(a), and the target angular rate, shown in Fig. 5.4(b) are significantly reduced using the sub-tether configuration, but are barely reduced from their initial condition using a single tether configuration. This demonstrates how using a sub-tether configuration 


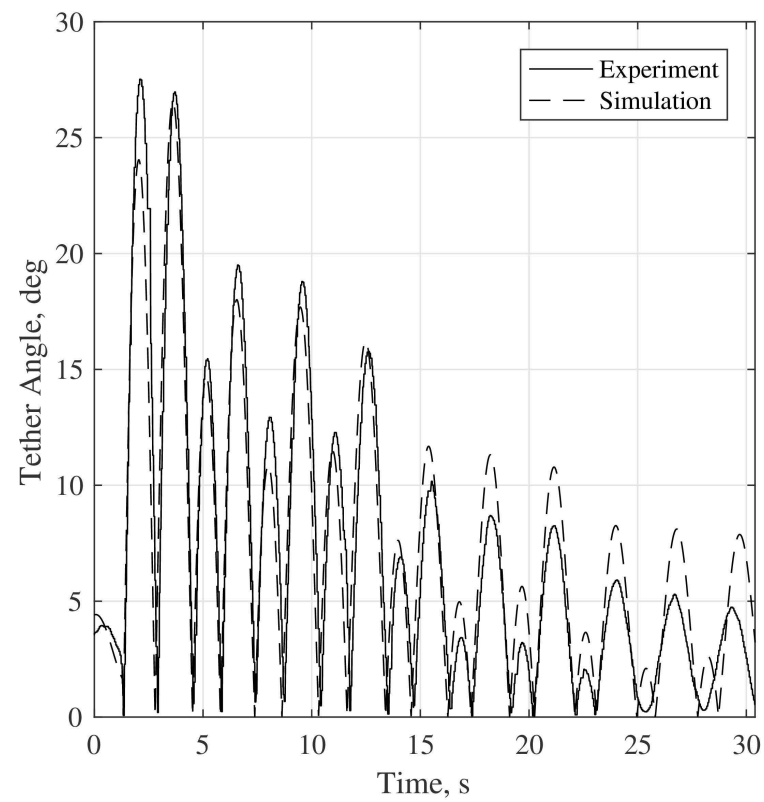

(a) Target tether angle

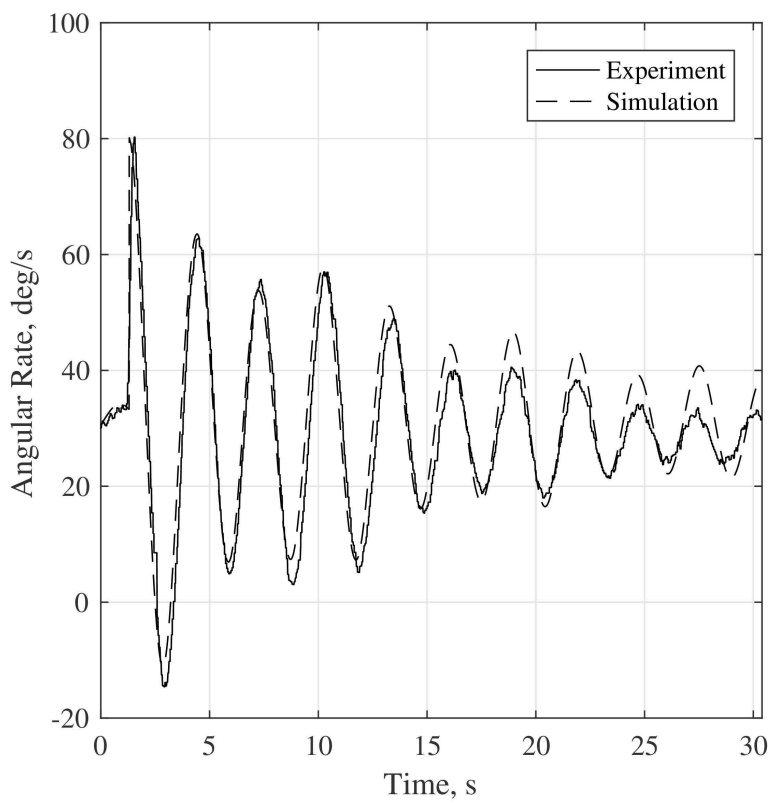

(b) Target angular rate

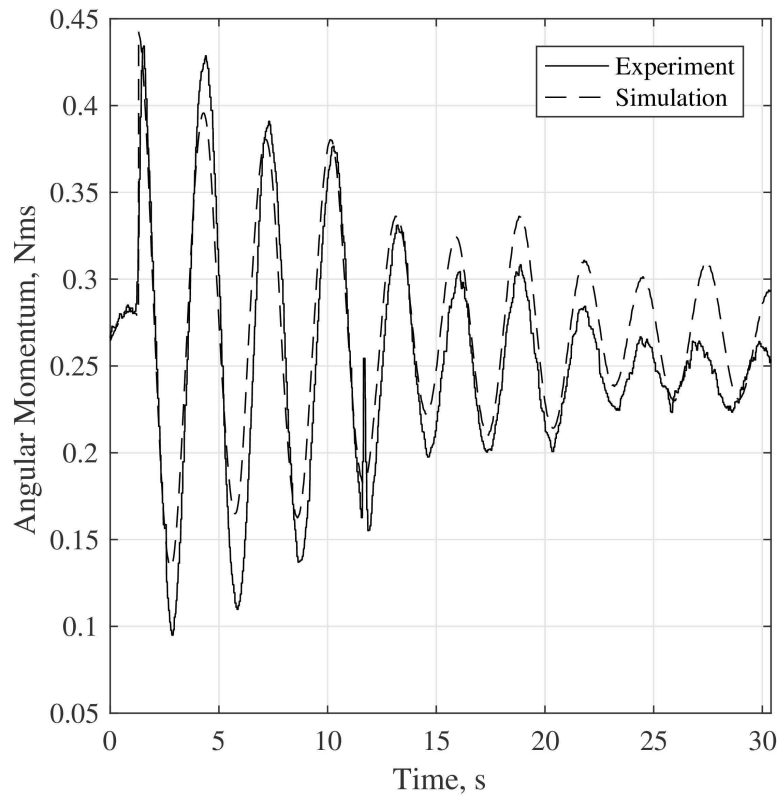

(c) Total angular momentum

Figure 5.3: Comparing experimental and simulated response for the planar TSS spin stabilization technique with sub-tethers.

passively enhances the stabilizing ability of the TSS. Based on the author's knowledge, these results constitute the first experimental results of using a chaser to regulate a tumbling target using tethers. 


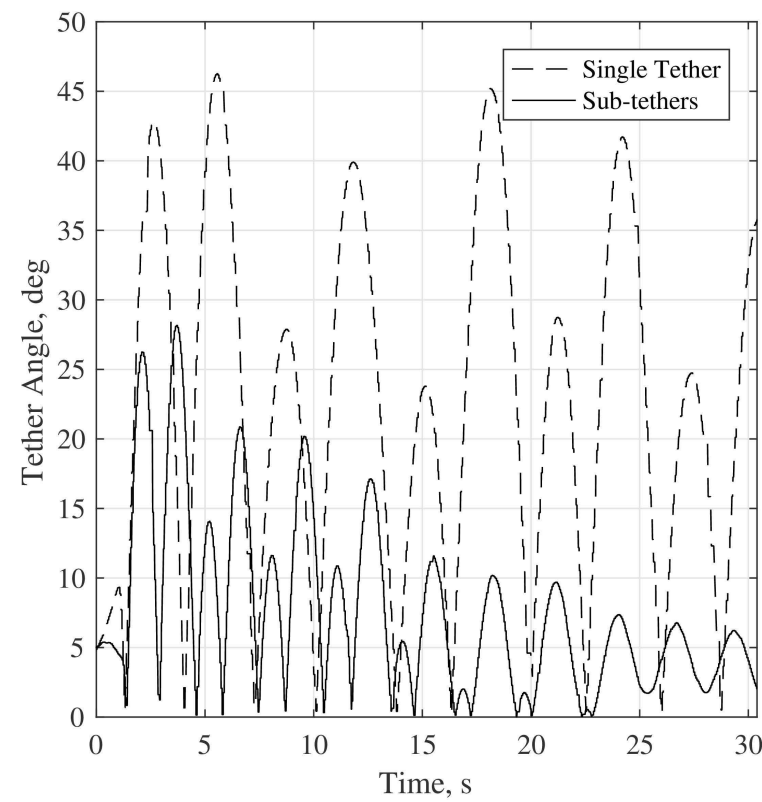

(a) Target tether angle

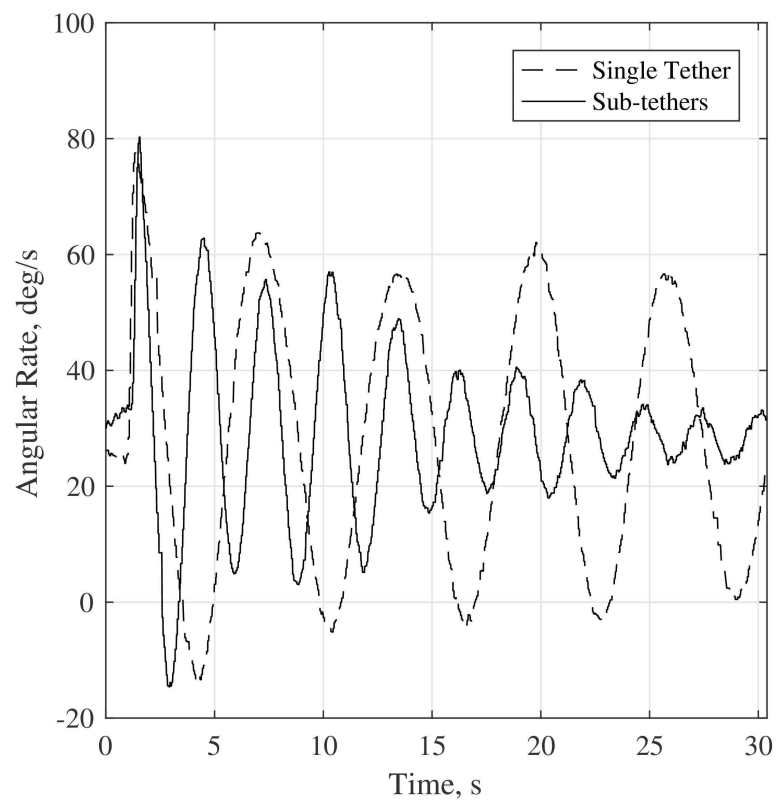

(b) Target angular rate

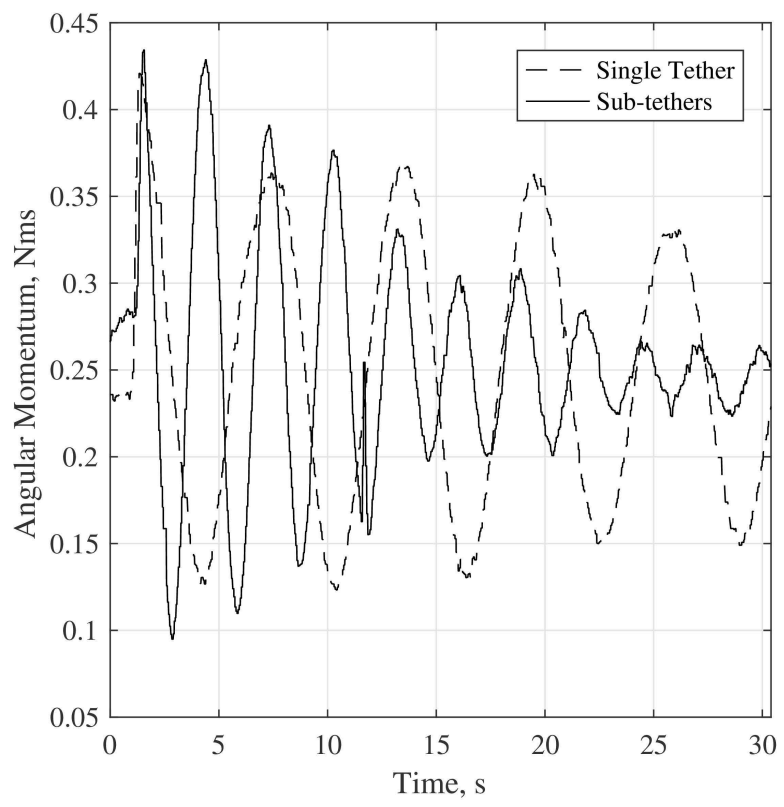

(c) Total angular momentum

Figure 5.4: Comparing planar experimental results of the two tether configurations when a TSS spin is used to regulate a tumbling target.

Experiments for the thrust stabilizing technique discussed in Sec. 4.3 .1 were performed, but abandoned due to the exhaust plumes from the chaser spacecraft interacting with the target which led to significant unmodeled effects. 


\section{Chapter 6}

\section{Conclusion}

In this chapter, a brief review of the motivation, research methodology, and results are presented. The contributions of this research to the field of space debris are discussed. Publications that resulted from this work are listed, practicality of the results are discussed, and possible future work is indicated.

\subsection{Thesis Summary}

Space debris is a growing concern for the world's major space agencies, due to the realization that cascaded debris collisions will render popular orbits too hostile to use. In order to prevent the exponential growth of the debris population, two things must be done. First, the creation of new debris must be limited. This task is currently underway, with regulations in place that require satellites to deorbit themselves within 25 years of the end of their life (although in practice this is not always followed). Second, active debris removal missions must remove high-risk debris from their orbits at a rate of 5-10 objects per year. The combination of these two measures will limit the growth of the space debris population.

The active debris removal strategy was considered in this thesis. Specifically, the study of tethered spacecraft systems. Tethered spacecraft systems arise when a flexible connection is created between two spacecraft. The most popular methods for establishing this connection is through the use of tethered nets or tethered harpoons. The creation of tethered spacecraft systems (i.e., the casting of a tethered net or launching of a harpoon) has been investigated by other researchers in the field. Once the debris is captured and stabilized, other researchers have investigated strategies to tow a piece of tethered debris out of orbit. However, the short period of time between these two stages, i.e., between when the spinning target is captured and when it is stabilized and ready for towing, is often neglected. This thesis bridges this gap by 
examining techniques for stabilizing spinning debris using tethers.

The main contribution of this thesis is a novel tethered spacecraft configuration, whereby the tether joining the two spacecraft is split into smaller sub-tether segments that attach to the target at various locations. In order to show the effectiveness of the proposed tether configuration, numerical simulations and experiments were performed that compare it to the presently-accepted single tether configuration.

To demonstrate the improvements that result from the proposed tether configuration, numerical simulations comparing the two tether configurations in identical scenarios were presented. First, the on-orbit dynamics of the tethered spacecraft system, including gravity-gradient torque, were presented. Simulation results show that the proposed sub-tether configuration enhances the ability of the tethered spacecraft system to stabilize a spinning debris compared to the single tether configuration. In addition, it was shown that the angular rate of the debris along the axis parallel to the tether is uncontrollable. Therefore, it is recommended that the chaser spacecraft approach the spinning debris along its axis of least angular momentum.

Next, it was desired to experimentally validate the numerical simulations on detumbling debris using tethers. In order to perform experimental validation, a laboratory facility first had to be designed and built. Air bearings were used to create a near friction-free environment on a large granite table, such that the dynamics experienced in space are nearly replicated in a planar laboratory environment. The author developed a number of significant elements used in the laboratory facility that includes: a power system, emergency stop system, floatation system, propulsion system, air refill system, a reaction wheel, data acquisition system, and a significant portion of the software. The end result is a laboratory facility that has two spacecraft platforms that can perform guidance, navigation, and control theories in simulations and experiments.

The on-orbit equations of motion were then redefined in a planar environment, so that planar simulations could be performed and compared to experiments performed in the planar laboratory facility. Planar simulations showed the same conclusions as the on-orbit numerical simulations. That is, that the proposed sub-tether configuration outperforms the presently-accepted single tether configuration. Planar 
experiments were then performed that showed excellent correlation with the planar simulations, validating the planar equations of motion. The discrepancies between the simulations and experiments were very small, and were attributed to the unmodeled effects present in the laboratory facility (friction, drag, signal noise). Another contribution of this research is that it presents the first, to the best of the author's knowledge, experiments that demonstrate detumbling a spinning debris using tethers.

\subsection{Significance of Work}

The significance of this work is demonstrated through the publications that have resulted from this research, as listed below:

\section{Conference Proceedings}

Hovell, K., and Ulrich, S., "Experimental Validation for Tethered Capture of Spinning Space Debris," AIAA Guidance, Navigation, and Control Conference, Grapevine, TX, 9-13 Jan, 2017, AIAA Paper 2017-1049 [47]. Best Presentation in Session Award.

Hovell, K., and Ulrich, S., "Attitude Stabilization of an Uncooperative Spacecraft in an Orbital Environment using Visco-Elastic Tethers," AIAA Guidance, Navigation, and Control Conference, San Diego, CA, 4-8 Jan, 2016, AIAA Paper 2016$0641[46]$.

Hovell, K., and Ulrich, S. "Attitude Stabilization of an Unknown and Spinning Target Spacecraft Using a Visco-Elastic Tether," 13th Symposium on Advanced Space Technologies in Robotics and Automation, Noordwijk, The Netherlands, 11-13 May, $2015[48]$.

\subsection{A Note on Practicality}

Sub-tethers were found to perform better at stabilizing a spinning debris. In practice, however, it is acknowledged that it may be more difficult to attach sub-tethers to the debris than a single tether. This section comments on the practicality of capturing a debris using a sub-tether configuration. It also discusses the usefulness of the TSS spin technique for stabilizing a debris. 
When casting a tethered net over a debris, it is feasible to attach the sub-tethers to the net at various locations. Then, as long as the net was properly deployed, the subtethers would effectively be attached to the debris at various locations. Therefore, capturing a debris with a net is a possible solution to establishing a sub-tethered connection with a debris.

Using harpoons to establish the tethered spacecraft system could cause difficulties. To establish the single tether configuration, a single harpoon would be launched into the target. However, to create a sub-tether configuration, multiple harpoons would have to be launched, at nearly the same time, in order to establish the tethered spacecraft system. In addition, all harpoons would have to embed in the target on the first attempt. If a subset of the harpoons become embedded in the target, it is not possible to reel back and redeploy the failed harpoons. Therefore, it is not feasible to use harpoons to capture a debris with sub-tethers.

The TSS spin technique to stabilize a spinning debris, while very effective at stabilizing a tumbling debris, poses challenges. Namely, how to deorbit the debris once it has been stabilized and the system is spinning. The author proposes that it would be possible to slowly despin the system, but maintaining a fast enough spin to maintain sufficient tension in the tethers to ensure control of the target. Then, a final, large, thrust could be used to end the TSS spin and initiate a towing scenario. It is possible that the TSS spin could be used to safely stabilize a debris with high angular rates before towing it out of orbit.

\subsection{Recommendations for Future Work}

Future work on the subject should focus on removing the assumption that the tethers were already rigidly attached to the spinning target. Desirably, there would be numerical simulations that show the casting of a net over a spinning target and then proceed to stabilize it with sub-tethers. These numerical simulations could then be validated experimentally.

Next, on-orbit experiments should be performed to test the technology in the six degree-of-freedom scenario present in orbit. This would remove the limitations present in the planar experimental facility at Carleton University. 
Additional work in the Spacecraft Proximity Operations Testbed consists of improving the control mixer, determining of the true thrust forces developed by each thruster, modeling thruster exhaust interaction with the other spacecraft, and incorporating the computer vision systems. 


\section{Bibliography}

[1] Shan, M., Guo, J., and Gill, E., "Review and Comparison of Active Space Debris Capturing and Removal Methods," Progress in Aerospace Sciences, Vol. 80, 2016, pp. 18-32.

[2] Kessler, D. J. and Cour-Palais, B. G., "Collision Frequency of Artificial Satellites - The Creation of a Debris Belt," Journal of Geophysical Research, Vol. 83, No. A6, 1978, pp. 2637-2646.

[3] Wormnes, K., Le Letty, R., Summerer, L., Krag, H., Schonenborg, R., DuboisMatra, O., Luraschi, E., Delaval, J., and Cropp, A., "ESA Technologies for Space Debris Remediation," 6th European Conference on Space Debris, Darmstadt, Germany, 2013.

[4] Kaplan, M. H., Boone, B., Brown, R., Criss, T. B., and Tunstel, E. W., "Engineering Issues for All Major Modes of In Situ Space Debris Debris Capture," AIAA Space 2010 Conference $\&$ Exposition, Anaheim, CA, 2010, AIAA paper 2010-8863.

[5] Persson, S. M. and Sharf, I., "Ground-Based Experiments Towards the Interception of Non-Cooperative Space Debris with a Robotic Manipulator," IEEE International Conference on Intelligent Robots and Systems, Hamburg, Germany, 2015 .

[6] Shah, S. V., Sharf, I., and Misra, A., "Reactionless Path Planning Strategies for Capture of Tumbling Objects in Space Using a Dual-Arm Robotic System," AIAA Guidance, Navigation, and Control Conference, Boston, MA, 2013, p. 18, AIAA paper 2013-4521.

[7] Reintsema, D., Thaeter, J., Rathke, A., Naumann, W., Rank, P., and Sommer, J., "DEOS - The German Robotics Approach to Secure and De-Orbit Malfunctioned Satellites from Low Earth Orbits," International Symposium on Artificial Intelligence, Robotics and Automation in Space, Sapporo, Japan, 2010.

[8] Hughes, P. C., Spacecraft Attitude Dynamics, Dover Publications, Mineola, NY, 2004, pp. 139-146, 93-99, 24-26, 232-238.

[9] Flores-Abad, A., Wei, Z., Ma, O., and Pham, K. D., "Optimal Control of Space Robots for Capturing a Tumbling Object with Uncertainties," Journal of Guidance, Control, and Dynamics, Vol. 37, No. 6, 2014, pp. 2014-2017. 
[10] Wen, H., Jin, D. P., and Hu, H. Y., "Advances in dynamics and control of tethered satellite systems," Acta Mechanica Sinica/Lixue Xuebao, Vol. 24, No. 3, 2008, pp. 229-241.

[11] Bonnal, C., Ruault, J. M., and Desjean, M. C., "Active Debris Removal: Recent Progress and Current Trends," Acta Astronautica, Vol. 85, 2013, pp. 51-60.

[12] Botta, E. M., Sharf, I., and Misra, A. K., "Contact Dynamics Modeling and Simulation of Tether Nets for Space-Debris Capture," Journal of Guidance, Control, and Dynamics, Vol. 40, No. 1, 2017, pp. 1-14.

[13] Dudziak, R., Tuttle, S., and Barraclough, S., "Harpoon Technology Development for the Active Removal of Space Debris," Advances in Space Research, Vol. 56, No. 3, 2015, pp. 509-527.

[14] Forshaw, J. L., Aglietti, G. S., Navarathinam, N., Kadhem, H., Salmon, T., Pisseloup, A., Joffre, E., Chabot, T., Retat, I., Axthelm, R., Barraclough, S., Ratcliffe, A., Bernal, C., Chaumette, F., Pollini, A., and Steyn, W. H., "RemoveDEBRIS: An in-orbit active debris removal demonstration mission," Acta Astronautica, Vol. 127, 2016, pp. 448-463.

[15] Ohkawa, Y., Kawamoto, S., Nishida, S., and Kitamura, S., "Research and Development of Electrodynamic Tethers for Space Debris Mitigation," Transactions of the Japan Society for Aeronautical and Space Sciences, Space Technology Japan, Vol. 7, No. ists26, 2009, pp. 5-10.

[16] Iki, K., Kawamoto, S., and Morino, Y., "Experiments and Numerical Simulations of an Electrodynamic Tether Deployment from a Spool-Type Reel Using Thrusters," Acta Astronautica, Vol. 94, No. 1, 2014, pp. 318-327.

[17] Johnson, L., Gilchrist, B., Estes, R., and Lorenzini, E., "Overview of Future NASA Tether Applications," Advances in Space Research, Vol. 24, No. 8, 1999, pp. 1055-1063.

[18] Wen, H., Jin, D., and Hu, H., "Three-dimensional deployment of electro-dynamic tether via tension and current control with constraints," Acta Astronautica, Vol. 129, No. September, 2016, pp. 253-259.

[19] Botta, E. M., Sharf, I., Misra, A. K., and Teichmann, M., "On the Simulation of Tether-Nets for Space Debris Capture with Vortex Dynamics," Acta Astronautica, Vol. 123, 2016, pp. 91-102.

[20] Benvenuto, R. and Lavagna, M., "Flexible Capture Devices for Medium To Large Debris Active Removal: Simulations Results To Drive Experiments," 12th Symposium on Advanced Space Technologies in Automation and Robotics, Noordwijk, The Netherlands, 2013. 
[21] Benvenuto, R. and Lavagna, M., "Net Capturing of Tumbling Space Debris: Contact Modelling Effects on the Evolution of the Disposal Dynamics," 13th Symposium on Advanced Space Technologies in Automation and Robotics, Noordwijk, The Netherlands, 2015.

[22] Benvenuto, R., Lavagna, M. R., and Salvi, S., "Multibody Dynamics Driving GNC and System Design in Tethered Nets for Active Debris Removal," Advances in Space Research, Vol. 58, No. 1, 2016, pp. 45-63.

[23] Shan, M., Guo, J., and Gill, E., "Deployment dynamics of tethered-net for space debris removal," Acta Astronautica, Vol. 132, 2017, pp. 293-302.

[24] Medina, A., Cercòs, L., Stefanescu, R., Benvenuto, R., Lavagna, M., Gonzalez, I., Rodriguez, N., and Ormnes, K., "Capturing Nets for Active Debris Removal: A Follow-Up on Microgravity Experiment Design To Validate Flexible Dynamic Models," 13th Symposium on Advanced Space Technologies in Automation and Robotics, Noordwijk, The Netherlands, 2015.

[25] Cercos, L., Stefanescu, R., Medina, A., Benvenuto, R., Lavagna, M., Gonzalez, I., Rodriguez, N., and Wormnes, K., "Validation of a Net Active Debris Removal Simulator Within Parabolic Flight Experiment," International Symposium on Artificial Intelligence, Robotics and Automation in Space, Montreal, Quebec, Canada, 2014.

[26] Lavagna, M., Armellin, R., Bombelli, A., Benvenuto, R., and Carta, R., "Debris Removal Mechanism Based on Tethered Nets," International Symposium on Artificial Intelligence, Robotics and Automation in Space, Turin, Italy, 2012.

[27] Aslanov, V. and Yudintsev, V., "Dynamics of Large Debris Connected to Space Tug by a Tether," Journal of Guidance, Control, and Dynamics, Vol. 36, No. 6, 2013, pp. 1654-1660.

[28] Aslanov, V. and Yudintsev, V., "Dynamics of Large Space Debris Removal Using Tethered Space Tug," Acta Astronautica, Vol. 91, 2013, pp. 149-156.

[29] Aslanov, V. S. and Yudintsev, V. V., "Behavior of Tethered Debris With Flexible Appendages," Acta Astronautica, Vol. 104, No. 1, 2014, pp. 91-98.

[30] Aslanov, V. S. and Yudintsev, V. V., "Dynamics, Analytical Solutions and Choice of Parameters for Towed Space Debris with Flexible Appendages," Advances in Space Research, Vol. 55, No. 2, 2015, pp. 660-667.

[31] Aslanov, V. S. and Yudintsev, V. V., "The Motion of Tethered Tug-Debris System with Fuel Residuals," Advances in Space Research, Vol. 56, No. 7, 2015, pp. 1493-1501. 
[32] Aslanov, V. S. and Ledkov, A. S., "Dynamics of Towed Large Space Debris Taking into Account Atmospheric Disturbance," Acta Mechanica, Vol. 225, No. 9, 2014, pp. 2685-2697.

[33] Aslanov, V. S., "Chaos Behavior of Space Debris During Tethered Tow," Journal of Guidance, Control, and Dynamics, Vol. 39, No. 10, 2016, pp. 2399-2405.

[34] Yudintsev, V. and Aslanov, V., "Detumbling Space Debris Using Modified Yo-Yo Mechanism," Journal of Guidance, Control, and Dynamics, published online 11 Jan. 2017.

[35] Zhang, F., Sharf, I., Misra, A., and Huang, P., "On-line estimation of inertia parameters of space debris for its tether-assisted removal," Acta Astronautica, Vol. 107, 2015, pp. 150-162.

[36] Huang, P., Wang, D., Meng, Z., Zhang, F., and Guo, J., "Adaptive Postcapture Backstepping Control for Tumbling Tethered Space RobotTarget Combination," Journal of Guidance, Control, and Dynamics, Vol. 39, No. 1, 2016, pp. 150-156.

[37] Mori, O. and Matunaga, S., "Formation and Attitude Control for Rotational Tethered Satellite Clusters," Journal of Spacecraft and Rockets, Vol. 44, No. 1, 2007, pp. 211-220.

[38] Cleary, S. and O'Connor, W. J., "Control of Space Debris Using an Elastic Tether and Wave-Based Control," Journal of Guidance, Control, and Dynamics, Vol. 39, No. 6, 2016, pp. 1392-1406.

[39] Mantellato, R., Lorenzini, E., Sternberg, D., Roascio, D., Saenz-Otero, A., and Zachrau, H., "Simulation of a tethered microgravity robot pair and validation on a planar air bearing," Acta Astronautica, 2016.

[40] Jasper, L. E. Z., Seubert, C. R., Schaub, H., Trushkyakov, V., and Yutkin, E., "Tethered tug for large low Earth orbit debris removal," Advances in the Astronautical Sciences, Vol. 143, 2012, pp. 2223-2242.

[41] Linskens, H. T. K. and Mooij, E., "Tether Dynamics Analysis and Guidance and Control Design for Active Space-Debris Removal," Journal of Guidance, Control, and Dynamics, Vol. 39, No. 6, 2016, pp. 1232-1243.

[42] Jasper, L. and Schaub, H., "Input Shaped Large Thrust Maneuver with a Tethered Debris Object," Acta Astronautica, Vol. 96, 2014, pp. 128-137.

[43] Jasper, L. and Schaub, H., "Tethered towing using open-loop input-shaping and discrete thrust levels," Acta Astronautica, Vol. 105, No. 1, 2014, pp. 373-384.

[44] Sabatini, M., Gasbarri, P., and Palmerini, G. B., "Elastic issues and vibration reduction in a tethered deorbiting mission," Advances in Space Research, Vol. 57, No. 9, 2016, pp. 1951-1964. 
[45] de Ruiter, A. H., Damaren, C., and Forbes, J. R., Spacecraft Dynamics and Control: An Introduction, Wiley, 2012.

[46] Hovell, K. and Ulrich, S., "Attitude Stabilization of an Uncooperative Spacecraft in an Orbital Environment using Visco-Elastic Tethers," AIAA Guidance, Navigation, and Control Conference, San Diego, CA, 2016, AIAA paper 2016-0641.

[47] Hovell, K. and Ulrich, S., "Experimental Validation for Tethered Capture of Spinning Space Debris," AIAA Guidance, Navigation, and Control Conference, Grapevine, TX, 2017, AIAA paper 2017-1049.

[48] Hovell, K. and Ulrich, S., "Attitude Stabilization of an Unknown and Spinning Target Spacecraft Using a Visco-Elastic Tether," 13th Symposium on Advanced Space Technologies in Robotics and Automation, Noordwijk, The Netherlands, 2015. 


\section{Appendix A}

\section{Laboratory Contributions}

This appendix provides, in fine detail, how all of the components developed by the author were designed, built, and implemented in the Spacecraft Proximity Operations Testbed. It is meant as a detailed reference for future students.

\section{A.1 Floatation System}

The floatation system used the components, per platform, shown in Table A.1. The plumbing used to route the air to the air bearings is presented in Section A.3.

The Mounting Screw is attached to the air bearing with the Mounting Clip. The mounting screw mates with the air bearing with a spherical joint, which allows the air bearing to align itself such that it is perfectly parallel with the granite table. The mounting screw is then attached to the base of the spacecraft platform using nuts and washers.

Clean and dry air is delivered to the air bearings through the Elbow Air Fittings. The air is regulated to 60 psi through the low pressure regulator. This pressure appears to be sufficient to float the platforms when they are roughly $20 \mathrm{~kg}$. It is difficult to measure the actual floating height, so these the pressure used was obtained by trial and error. The top pressure gauge on the side of the platform reads the pressure delivered to the air bearings. If more pressure is needed in the future, simply

Table A.1: Part list for the floatation system.

\begin{tabular}{cccc}
\hline Name & Manufacturer (Supplier if different) & Part Number & Quantity \\
\hline Air Bearing & New Way Air Bearings & S104001 & 3 \\
Elbow Air Fitting & New Way Air Bearings & S90F049 & 3 \\
Mounting Screw & New Way Air Bearings & S8013B17 & 3 \\
Mounting Clip & New Way Air Bearings & S8013S01 & 3 \\
Solenoid Valve & Peter Paul (Sempress Pneumatics) & 582W19DGM 12VDC & 1 \\
\hline
\end{tabular}


increase the pressure to the air bearings by adjusting the low pressure regulator. Note: DO NOT EXCEED 80 PSI. Figure A.1 shows the air bearing, mounting screw, mounting clip, air supply line, and elbow air fitting. Figure A.2 shows the top and bottom of the air bearings on their own.

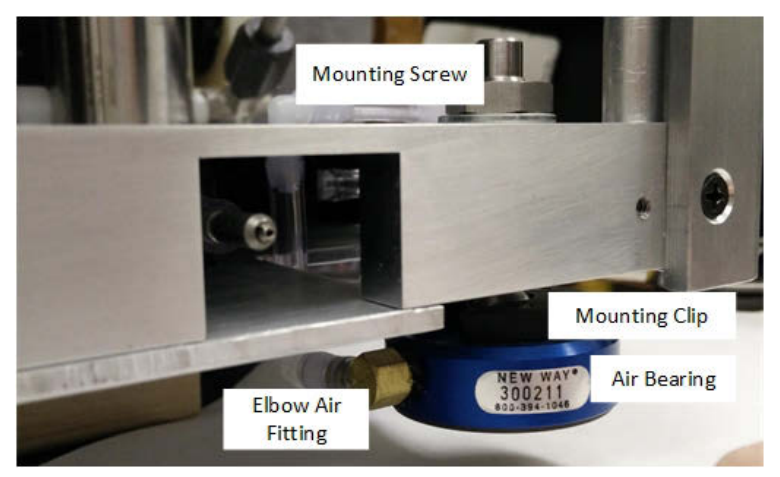

Figure A.1: Air bearing mounted to the spacecraft platform. The blue air bearing is fed air through the flexible clear hose, which connects to the bearing through the Elbow Air Fitting. A mounting screw attaches the bearing to the base of the platform. The mounting clip secures the mounting screw to the bearing.

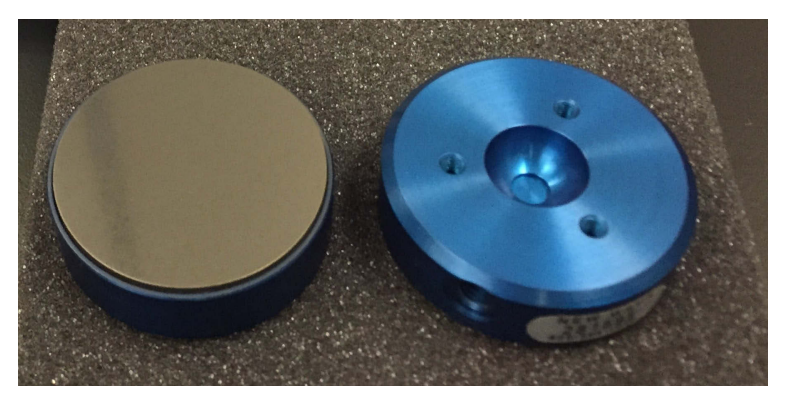

Figure A.2: Bottom of air bearing is shown on the left. The top and mounting clip holes are shown on the right. The spherical groove is where the mounting screw sits.

\section{A.2 Propulsion System}

The propulsion system passes compressed air through nozzles to generate thrust to allow the spacecraft platform to translate and rotate on the table. Air at 80 psi, delivered from the plumbing system discussed in Sec. A.3, is expelled through air nozzles. At this pressure, the air nozzles are rated to produce $1.0 \mathrm{~N}$ of thrust. However, in 
Table A.2: Part list for the propulsion system.

\begin{tabular}{cccc}
\hline Name & Manufacturer (Supplier if different) & Part Number & Quantity \\
\hline Air Nozzle & Silvent & MJ4 & 8 \\
Solenoid Valve & Peter Paul (Sempress Pneumatics) & 582W19DGM 12VDC & 8 \\
\hline
\end{tabular}

practice it appears that this value is near $0.25 \mathrm{~N}$. An experimental setup to accurately determine the thrust produced by each thruster is being developed by another student. The components specific to the propulsion system are listed in Table A.2

The nozzle is attached to the output of the solenoid valve, using some spare tubing. Figure A.3 shows the solenoid valve and nozzle assembly.



Figure A.3: Solenoid valve attached to air nozzle. Note: This is not the typical solenoid valve, but the connection is identical.

It is desirable to have the solenoid valve very close to the nozzle, such that when the valve is closed, only a small amount of air remains in the lines that is expelled as residual thrust. The thrusters are mounted with hot glue to the underside of the bus deck. Small cutouts have been machined into the bus deck where the nozzles expel their exhaust, as shown in Figure A.4.

The plumbing used to deliver the 80 psi air to the nozzles is discussed in Section A.3. The electronics used to actuate the solenoid valves is discussed in Section A.4. 


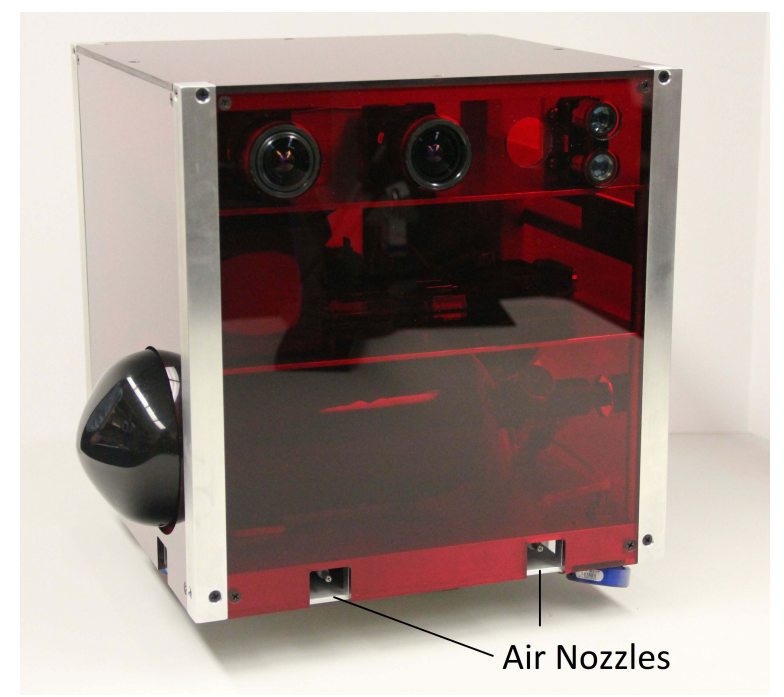

Figure A.4: Air nozzle holes in the spacecraft structure.

\section{A.3 Plumbing}

Compressed air at 4500 psi is stored in small air tanks that are typically used for paintball/air gun applications. The method for filling the tanks is discussed in Sec. A.5. This section discusses how the air from the tank is regulated and delivered to the air bearings and thrusters. The components used are listed in Table A.3.

Air is released from the air tank through the tank adapter, and flows through stainless steel tubing at 800 psi to the high pressure regulator, which regulates it to $80 \mathrm{psi}$, and is shown on the bottom pressure gauge. After the high pressure regulator, the air flows past a pressure relief valve that vents the lines if the pressure exceeds 100 psi (in the event that the high pressure regulator fails). Following this, the line is split. One line goes to the air bearings, and the other goes to the thrusters. The air bearing branch passes through a low pressure regulator which brings the pressure to 60 psi for the air bearings. The air then passes through an air filter to remove any "last minute" contaminants. Following this, the air passes through the air bearing solenoid valve and then branches off to the bearings themselves. The thruster branch immediately goes to the underside of the bus deck, where it branches off to all 8 solenoid valves.

Many fittings are used. Figure A.5 shows the air flow from the top of the bus deck, and Fig. A.6 shows the air flow from the underside of the bus deck. 
Table A.3: Part list for the plumbing system.

\begin{tabular}{|c|c|c|c|}
\hline Name & $\begin{array}{c}\text { Manufacturer } \\
\text { (Supplier if different) }\end{array}$ & Part Number & Quantity \\
\hline Air Tank & Guerrilla & B00B1LOS3C & 1 \\
\hline Air Tank Adapter & Ansgear.com & $\begin{array}{l}\text { EMPIREUFA- } \\
\text { BLACK }\end{array}$ & 1 \\
\hline $\begin{array}{l}\text { Steel Tubing to M } \\
1 / 8 " \text { NPT }\end{array}$ & $\mathrm{MMC}$ & $52215 \mathrm{~K} 439$ & 1 \\
\hline 1/4" Steel Tubing & MMC & 89895K411 & 2" \\
\hline Steel Tubing Cutter & MMC & 2751A12 & 1 \\
\hline $\begin{array}{l}\text { Steel Tubing to M } \\
1 / 8 \text { " NPT Elbow }\end{array}$ & MMC & $52245 \mathrm{~K} 545$ & 1 \\
\hline Teflon Tape & MMC & $6802 \mathrm{~K} 11$ & 1 \\
\hline $\begin{array}{l}\text { High Pressure } \\
\text { Regulator }\end{array}$ & Swagelok & KCP1FLC2A2P10000 & 1 \\
\hline $\begin{array}{l}\text { Pressure Relief Valve } \\
\qquad(100 \mathrm{psi})\end{array}$ & $\mathrm{MMC}$ & $5784 \mathrm{~T} 11$ & 1 \\
\hline $\begin{array}{c}\text { Pressure Relief Valve } \\
\text { Adapter }\end{array}$ & $\mathrm{MMC}$ & $4464 \mathrm{~K} 351$ & 1 \\
\hline $\begin{array}{l}\text { Low Pressure } \\
\text { Regulator }\end{array}$ & MMC & $3834 \mathrm{~T} 51$ & 1 \\
\hline $\begin{array}{l}\text { Secondary Air Filter } \\
\text { Pressure Gauge }\end{array}$ & $\mathrm{MMC}$ & $41575 \mathrm{~K} 61$ & 1 \\
\hline $\begin{array}{l}\text { 0-160 psi (Nozzle } \\
\text { line) }\end{array}$ & $\mathrm{MMC}$ & $4000 \mathrm{~K} 718$ & 1 \\
\hline $\begin{array}{c}\text { Pressure Gauge } \\
0-100 \text { psi (Air } \\
\text { Bearing line) }\end{array}$ & $\mathrm{MMC}$ & $4000 \mathrm{~K} 718$ & 1 \\
\hline $\begin{array}{l}\text { Clear PVC Tubing } \\
1 / 8 " \text { PVC Tubing }\end{array}$ & MMC & $1487 \mathrm{~T} 1$ & $50 \mathrm{ft}$ \\
\hline $\begin{array}{c}\text { Tee with } 1 / 8 " \mathrm{M} \\
\text { NPT }\end{array}$ & MMC & $5121 \mathrm{~K} 941$ & 1 \\
\hline $\begin{array}{l}\text { 1/8" PVC Tubing to } \\
1 / 8 " \mathrm{M} \mathrm{NPT}\end{array}$ & MMC & $2808 \mathrm{~K} 22$ & 2 \\
\hline $\begin{array}{l}\text { 1/8" PVC Tubing } \\
\text { Elbow }\end{array}$ & MMC & $2808 \mathrm{~K} 115$ & 13 \\
\hline $\begin{array}{c}\text { 1/8" PVC Tubing } \\
\text { Tee }\end{array}$ & $\mathrm{MMC}$ & $5117 \mathrm{~K} 13$ & 9 \\
\hline $\begin{array}{l}\text { 1/8" PVC Tubing to } \\
1 / 8 " \text { F NPT }\end{array}$ & MMC & $5121 \mathrm{~K} 511$ & 2 \\
\hline $\begin{array}{c}\text { 1/8" PVC Tubing to } \\
\text { M 10-32 }\end{array}$ & MMC & $5117 \mathrm{~K} 83$ & 20 \\
\hline $\begin{array}{c}\text { 1/8" PVC Tubing } \\
\text { Cross }\end{array}$ & $\mathrm{MMC}$ & $5463 \mathrm{~K} 93$ & 1 \\
\hline
\end{tabular}




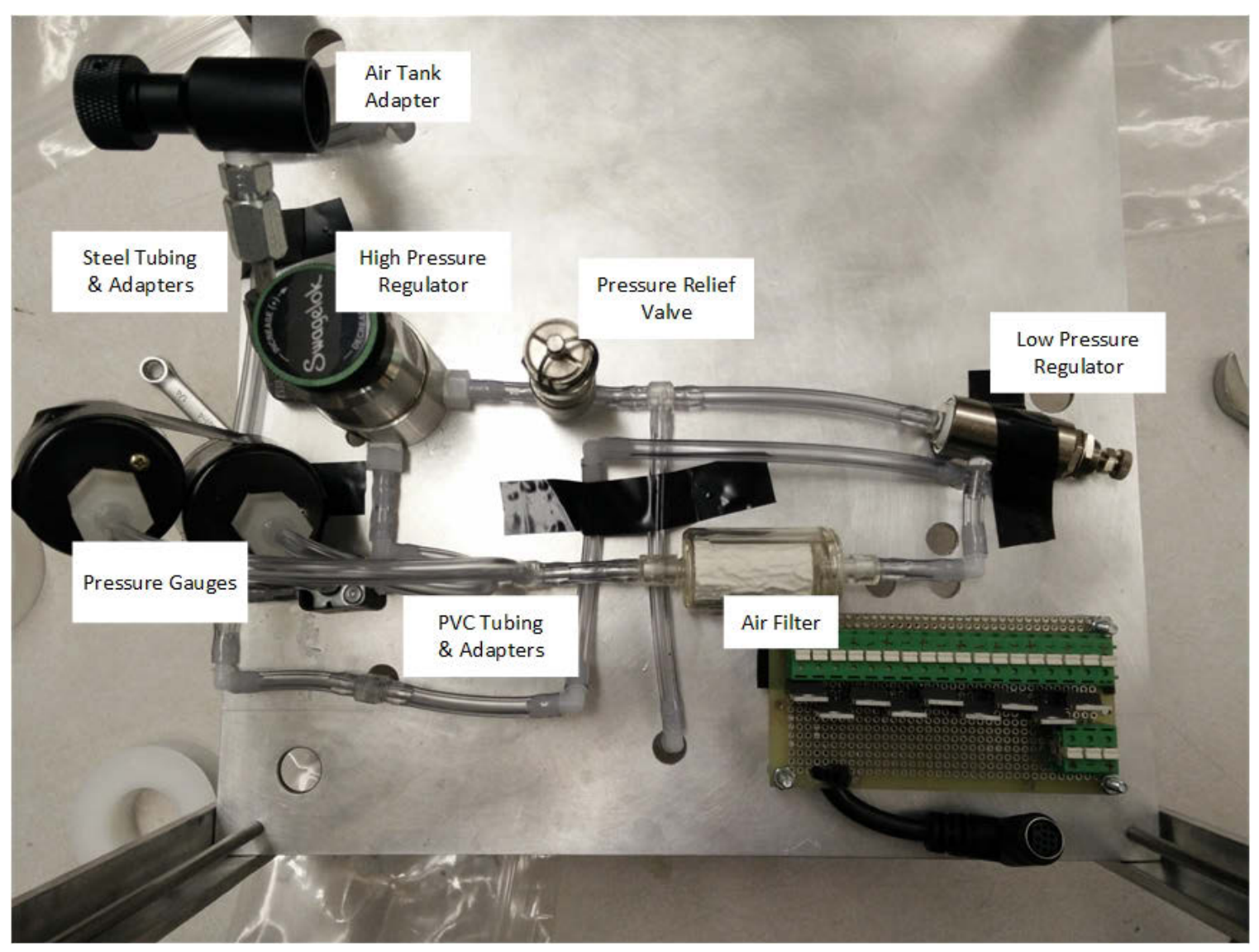

Figure A.5: Air flow on the top side of the bus deck.

\section{A.3.1 Using PVC Fittings}

The flexible PVC tubing is attached to the various components using barbed fittings. Barbed fittings have a small raised edge that prevents the hose from coming detached from the fitting, shown in figure A.7.

The procedure for attaching the PVC tubing to a fitting is as follows:

1. Select the fitting you wish to use.

2. Cut a segment of tubing to the length required, using the wire cutters.

3. Apply some "Amazing Goop" to the fitting, making sure to apply this to only the exterior of the fitting, as shown in Figure A.8(a).

4. Manually push the segment of tubing onto the fitting. Use pliers if necessary, as shown in Figs. A.8(b) and A.8(c). 


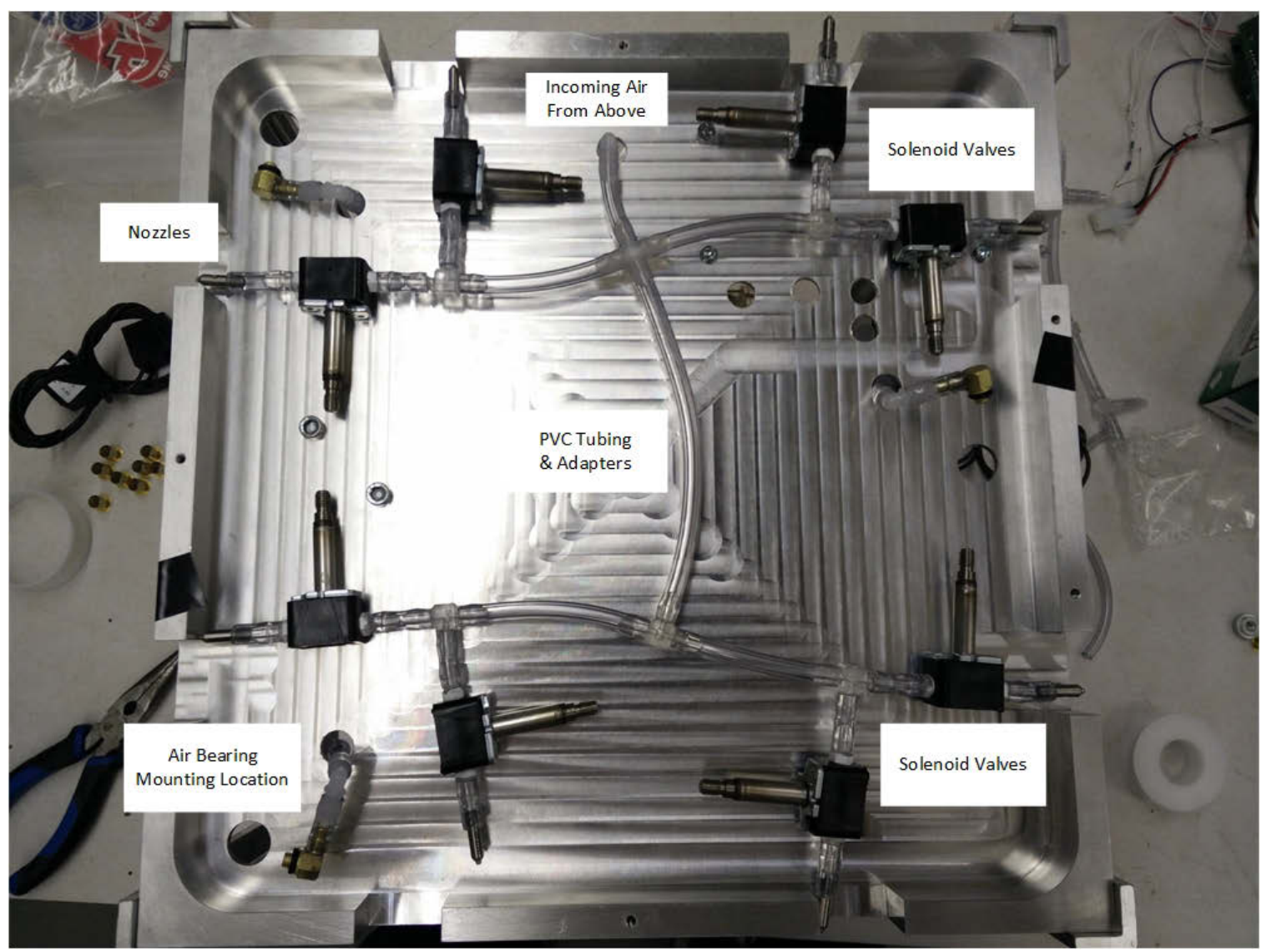

Figure A.6: Air flow on the underside of the bus deck.

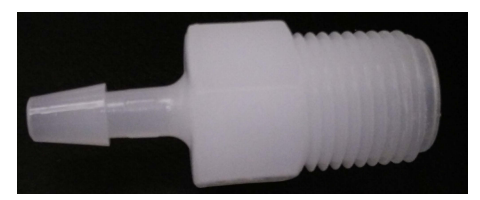

Figure A.7: A barbed fitting for use with the PVC tubing.

5. Allow the "Goop" to cure for 24 hours before supplying air.

6. To remove a tube from a fitting, the hose will be destroyed. Use the wire cutters to cut away at the hose, without damaging the fitting.

Note: Use teflon tape for all threaded fittings.

A schematic of the air flow is shown in Figure A.9. 


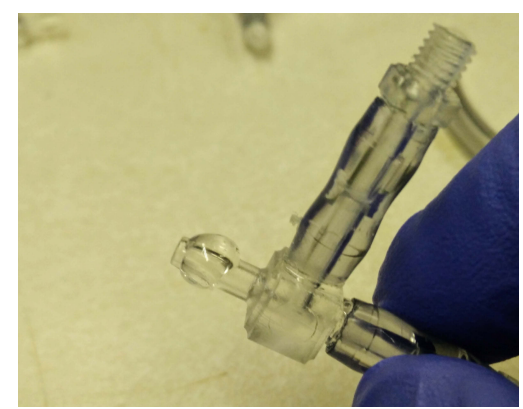

(a) "Amazing Goop" applied to the exterior of the fitting

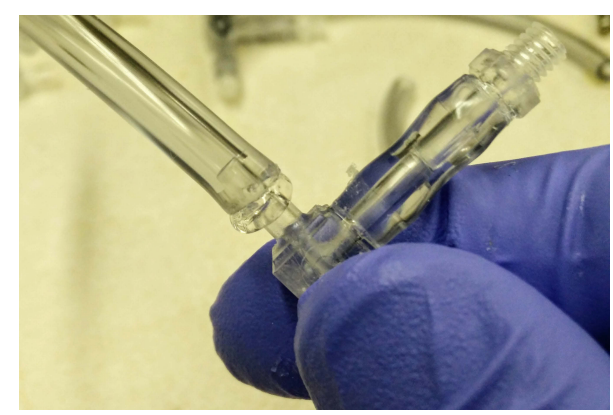

(b) PVC tubing pushed on the fitting.

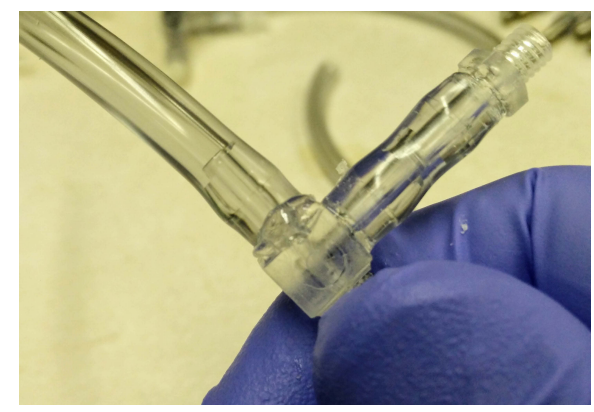

(c) PVC tubing fully secured on the fitting.

Figure A.8: The process of attaching PVC tubing to a fitting.

\section{A.4 Solenoid Valve Actuation}

To actuate the solendoid valves, MOSFET power transistors are used. MOSFETs have an extremely fast response time, and are very inexpensive. One MOSFET is used for each solenoid valve. Table A.4 lists the components used in the design of the solenoid control board. Figure A.10 shows the solenoid control board.

The digital signals from the on-board computer come to the transistor board through the signal cable on the right. The multicoloured wires run to the gate of each transistor. When the on-board computer applies a $+3.3 \mathrm{~V}$ signal to the gate (the ground of the computer is connected to the source of the MOSFET), the GateSource Threshold voltage $(1.7 \mathrm{~V})$ is passed, and the MOSFET allows current to flow with nearly zero resistance. Through this mechanism, the MOSFET acts as a switch that is activated by the on-board computer.

In case of a MOSFET failure, it is desirable to protect the on-board computer 


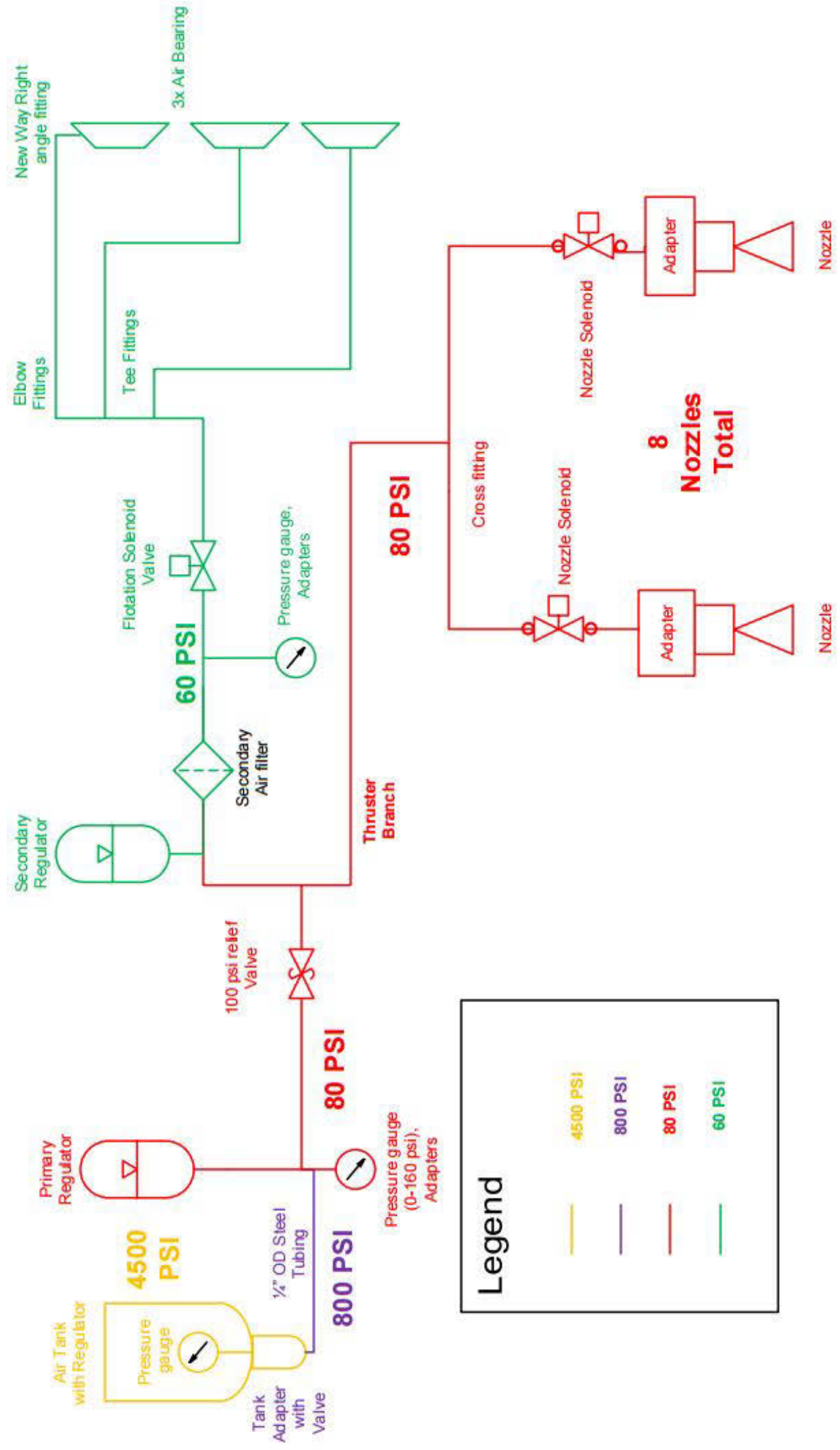

Figure A.9: Air flow diagram responsible for the on board propulsion and floatation systems. 
Table A.4: Part list for the transistor board.

\begin{tabular}{cccc}
\hline Name & Manufacturer (Supplier if different) & Part Number & Quantity \\
\hline Transistors & NXP Semiconductors (Digikey) & 568-7512-5-ND & 9 \\
Push Terminals & TE Connectivity (Digikey) & A104596-ND & 21 \\
Signal Cable F & Tensility (Digikey) & 839-1103-ND & 1 \\
Signal Cable M & Tensility (Digikey) & 839-1054-ND & 1 \\
$10 \mathrm{k} \Omega$ resistors & & & 9 \\
$22 \mathrm{k} \Omega$ resistors & & & 9 \\
Breadboard & & & 1 \\
\hline
\end{tabular}

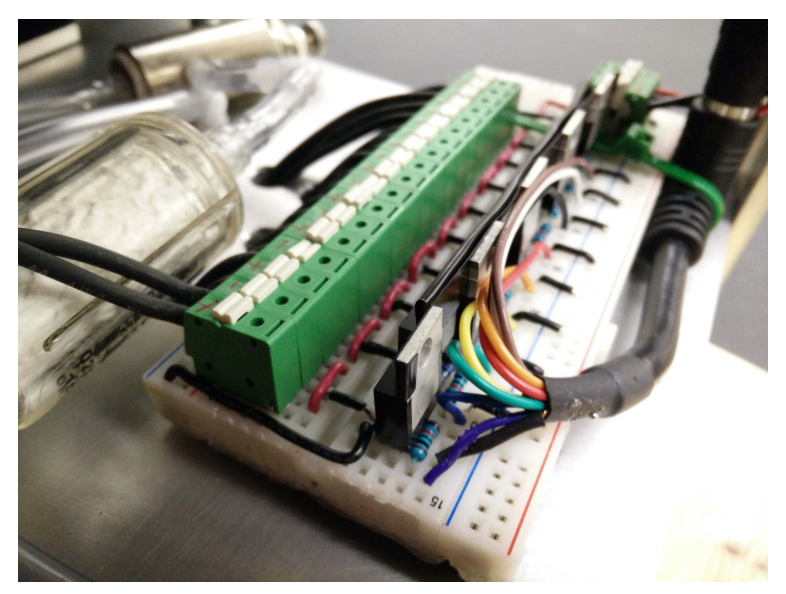

Figure A.10: The board of MOSFETs used to control the solenoid valves.

from the $12 \mathrm{~V}$ line. Therefore, a $10 \mathrm{k} \Omega$ resistor is placed in series with the signal cable. Pulldown resistors of $22 \mathrm{k} \Omega$ are placed between the gate and the source pins, in order to allow the electrons to flow to ground when the on-board computer sends a Low signal.

Power flows through the solenoid and transistor circuitry as follows:

1. $+5 \mathrm{~V}$ and GND leave the $5 \mathrm{~V}$ regulator and power the on-board computer.

2. $+12 \mathrm{~V}$ leaves the $12 \mathrm{~V}$ regulator and immediately goes to all solenoids.

3. The MOSFETs are downstream of the solenoids. Therefore, when current is not allowed to flow, there is a $12 \mathrm{~V}$ drop across the MOSFET. However, when current is allowed to flow, the $12 \mathrm{~V}$ drop is across the solenoid valve which causes it to open.

4. The source pin of the MOSFETs is connect to GND. 
5. The on-board computer supplies the Gate with 0 or $+3.3 \mathrm{~V}$ with respect to GND. When the High signal is supplied, the MOSFET acts as a short, and when the Low signal is applied, the MOSFET acts as a break in the circuit.

It should be noted that the source pins of all the MOSFETs used for switching the solenoids pass through another MOSFET before reaching the GND terminal on the $12 \mathrm{~V}$ regulator. This additional MOSFET is used for emergency stop purposes, discussed in Sec. A.8. The air bearing MOSFET is exempt from the emergency stop circuit.

\section{A.5 Air Refill System}

A self contained system to pressurize the air tanks with 4500 psi air was developed. This system was preferred to purchasing high pressure cylinders, because a constant repurchase cycle of high pressure air was deemed undesirable.

The air tanks used are typically used for paintball or air guns. Therefore, an air compressor typically purchased by enthusiasts was acquired for the lab. However, the air used for air guns does not have as strict requirements on cleanliness compared to the air bearings, so some conditioning of the air was required.

The air is passed through a dessicant dryer. This removes any moisture from the air. The dryer used is made for air guns, so all fittings were appropriate. The air is then passed through a filter that removes particles and oil from the air. Since the filter is not typically used with air guns, certain fittings had to be purchased to allow for all the tubing to connect together. Finally, the air enters the storage tank through a quick-connect valve.

Typically, users fill the air tank and then vent the lines through the pressure relief valve on the air compressor. However, in our application this is not acceptable. The filter in the system has a one-way valve that can break if there is greater than a 100 psi drop across the valve. Therefore, it is necessary to vent the lines downstream of the filter. For this reason, a needle valve was purchased and attached downstream of the filter such that the system can be safely vented.

A list of the required and achieved air quality specifications is shown in Table A.5. A list of the components is shown in Table A.6. 
Table A.5: Desired and achieved air quality.

\begin{tabular}{ccc}
\hline Parameter & Minimum Quality & Achieved Quality \\
\hline Max Particle Size $(\mu \mathrm{m})$ & 5.0 & 1.0 \\
Dewpoint $\left({ }^{\circ} \mathrm{F} @ 100 \mathrm{psi}\right)$ & -4 & Unknown \\
Oil Content $\left(\mathrm{mg} / \mathrm{m}^{3}\right)$ & 1.0 & 0.03 \\
\hline
\end{tabular}

Table A.6: Part list for the air refill system.

\begin{tabular}{|c|c|c|c|}
\hline Name & $\begin{array}{c}\text { Manufacturer } \\
\text { (Supplier if different) }\end{array}$ & Part Number & Quantity \\
\hline Air Compressor & $\begin{array}{l}\text { Airguns of Arizona } \\
\text { (Airgunsource.com) }\end{array}$ & $\begin{array}{l}\text { Omega Super } \\
\text { Charger }\end{array}$ & 1 \\
\hline Air Dryer & $\begin{array}{c}\text { Airguns of Arizona } \\
\text { (Airgunsource.com) } \\
\text { Parker Finite }\end{array}$ & $\begin{array}{l}\text { Diablo Dry Air } \\
\text { System }\end{array}$ & 1 \\
\hline High Pressure Filter & $\begin{array}{c}\text { (Ottawa Compressor } \\
\text { Service) }\end{array}$ & JN1A-4CN & 1 \\
\hline $\begin{array}{l}\text { M } 1 / 4 " \text { to } F 1 / 8 " \\
\text { NPT adapter }\end{array}$ & $\mathrm{MMC}$ & $48805 \mathrm{~K} 523$ & 1 \\
\hline $\begin{array}{c}\text { M } 1 / 4 " \text { to } \mathrm{M} 1 / 8 " \\
\text { NPT adapter }\end{array}$ & $\mathrm{MMC}$ & $48805 \mathrm{~K} 86$ & 1 \\
\hline Fill Hose & Airguns of Arizona & Fill Hose & 2 \\
\hline Needle Bleed Valve & MMC & $7833 \mathrm{~K} 85$ & 1 \\
\hline $\begin{array}{l}\text { High Pressure Tee } \\
\text { (to bleed valve) }\end{array}$ & $\mathrm{MMC}$ & $48805 \mathrm{~K} 124$ & 1 \\
\hline $\begin{array}{l}\text { High Pressure Tee } \\
\text { (to pressure gauge) }\end{array}$ & MMC & 48805K 98 & 1 \\
\hline Pressure Gauge & $\mathrm{MMC}$ & $4089 \mathrm{~K} 61$ & 1 \\
\hline
\end{tabular}


Table A.7: Part list for the reaction wheel.

\begin{tabular}{|c|c|c|c|}
\hline Name & $\begin{array}{l}\text { Manufacturer } \\
\text { (Supplier) }\end{array}$ & Part Number & Quantity \\
\hline Wheel & $\begin{array}{l}\text { Author \& Machine } \\
\text { Shop Staff }\end{array}$ & rwa_wheel.sldprt & 1 \\
\hline Support Cylinder & $\begin{array}{l}\text { Author \& Machine } \\
\text { Shop Staff }\end{array}$ & rwia_top_tube.sldprt & 1 \\
\hline Base Plate & $\begin{array}{l}\text { Author \& Machine } \\
\text { Shop Staff }\end{array}$ & rwia_bottom_plate.sldprt & 1 \\
\hline Bearing Holder & $\begin{array}{c}\text { Author \& Machine } \\
\text { Shop Staff }\end{array}$ & rwia_top_plate.sldprt & 1 \\
\hline $\begin{array}{l}\text { Motor to Wheel } \\
\text { Adapter }\end{array}$ & $\begin{array}{l}\text { 3D printed in MAE } \\
\text { Department }\end{array}$ & rwia_adapter.sldprt & 5 \\
\hline Thrust Bearing & $\begin{array}{l}\text { NTN (General } \\
\text { Bearing Service) }\end{array}$ & 51104 & 1 \\
\hline Motor & $\begin{array}{l}\text { Maxon Motors } \\
\text { (Electromate) }\end{array}$ & 339260 & 1 \\
\hline Gearbox & $\begin{array}{l}\text { Maxon Motors } \\
\text { (Electromate) }\end{array}$ & 143980 & 1 \\
\hline Motor Controller & $\begin{array}{l}\text { Maxon Motors } \\
\text { (Electromate) }\end{array}$ & 466023 & 1 \\
\hline Flexprint Adapter & Molex & $52207-1133$ & 1 (10 free samples) \\
\hline Breakout Board & Adafruit & 1325 & 1 \\
\hline
\end{tabular}

The extreme high pressure system has had three failures thus far. It is important to learn from the failures and continually improve the system. The air compressor has failed twice. A contact at Airguns of Airzona (Todd DeDrio) is extremely knowledgeable about the air compressor and is the main point of contact when troubles are encountered. Contact the author for his contact information. He will walk you through the steps necessary to repair the compressor.

\section{A.6 Reaction Wheel}

A reaction wheel was built for attitude control purposes. Due to the limited budget of the lab, a reaction wheel was designed and built in-house. Table A.7 lists the parts that were machined or purchased to construct the reaction wheel.

Drawings of the machined parts are available in "SPOT/Subsystems/Reaction 
Wheel/Drawings". The design details are in the "Design.xlsx" document the "Reaction Wheel" folder, and are summarized as follows:

- A resultant angular rate of $1 \mathrm{rpm}$ on the platform due to the reaction wheel was desired.

- The platform inertia was estimated to be $0.96 \mathrm{~kg} \cdot \mathrm{m}^{2}$ at the time the reaction wheel design was begun.

- A large reaction wheel spinning at a lower speed was more desirable than a small reaction wheel spinning at a high speed. This is because the force associated with an unbalanced wheel increases with the square of the wheel's angular rate. Therefore, a slow wheel has a less strict balancing requirement than a fast one. Since access to a wheel balancing facility was not readily available, spinning a large wheel slowly was the approach herein taken.

- The maximum allowable dimensions of the wheel were determined to be 4.4 inches in diameter and 2.4 inches tall due to space constraints on the platform. This yields a wheel inertia of $0.0074 \mathrm{~kg} \cdot \mathrm{m}^{2}$.

- In order for the platform to spin at $1 \mathrm{rpm}$, the wheel must spin at $129 \mathrm{rpm}$. This drives the selection of an appropriate motor and gearbox.

- A Maxon brushless motor, with a no load speed of $7950 \mathrm{rpm}$ was selected. It was to be operated at a maximum speed of $7000 \mathrm{rpm}$. With a gearbox with a reduction of 53:1, this would yield a maximum wheel speed of $128 \mathrm{rpm}$.

- A controller was chosen to perform closed loop feedback control on the motor. It was setup in rate control mode, and it uses feedback from Hall sensors embedded in the motor. A pulse width modulated signal from the on-board computer tells the control board the desired wheel speed. Escon Studio software on a laptop can be used to modify settings on the control board.

The items were then purchased, machined, and tested. The following paragraph shows performance parameters and then discusses suggested changes for future designs. 
The reaction wheel was connected to the Raspberry Pi 3 as well as the author's laptop. The control board performance can be monitored in real time using the Maxon motor "ESCON Studio" software. Using this, the Pi 3 sent speed commands to the controller, and the Hall sensor feedback signal was recorded to determine what speed was achieved. It was found that the motor reached the desired speed to within $10 \mathrm{rpm}$ over the range [0 7000] rpm. By varying the desired speed of the motor over time, any torque within the capabilities of the motor can be achieved. This was tested, and any torque could accurately be obtained to within $0.1 \%$ of the desired torque, up to a maximum torque of $0.1 \mathrm{Nm}$. Therefore, the reaction wheel's performance was demonstrated to be as expected when commanded via the Pi 3 computer.

The limiting factor in the motor speed and torque is driven by the supplied voltage. It was learned, after the components were purchased, that a $12 \mathrm{~V}$ motor actually requires a larger than $12 \mathrm{~V}$ power supply. An equation in the motor controller manual dictates the required supply voltage to achieve a desired speed and torque. With the limited $12 \mathrm{~V}$ supply already incorporated in the design, the speed-load curve shown in Figure A.11 was generated. The figure shows that above $7000 \mathrm{rpm}$, the maximum torque is negative, indicating that the maximum speed possible with the available power supply is roughly $7000 \mathrm{rpm}$. Therefore, the motor speed is capped at 7000 rpm, to ensure the accuracy of the commanded values.

In practice, the RED platform achieved $3 \mathrm{rpm}$ due to the reaction wheel. This additional performance was attributed to the actual platform inertia being roughly $1 / 3$ of the predicted inertia.

In the future, it is recommended to purchase a $9 \mathrm{~V}$ motor, so that the $12 \mathrm{~V}$ power supply available will be able to drive the motor to its full range of speeds and torques. It is also suggested to increase the speed of the wheel by up to a factor of 5 , due to the success in machining a pre-balanced reaction wheel. This would give 5 times the angular momentum capacity and possibly allow for $15 \mathrm{rpm}$ angular rates on the platform. 


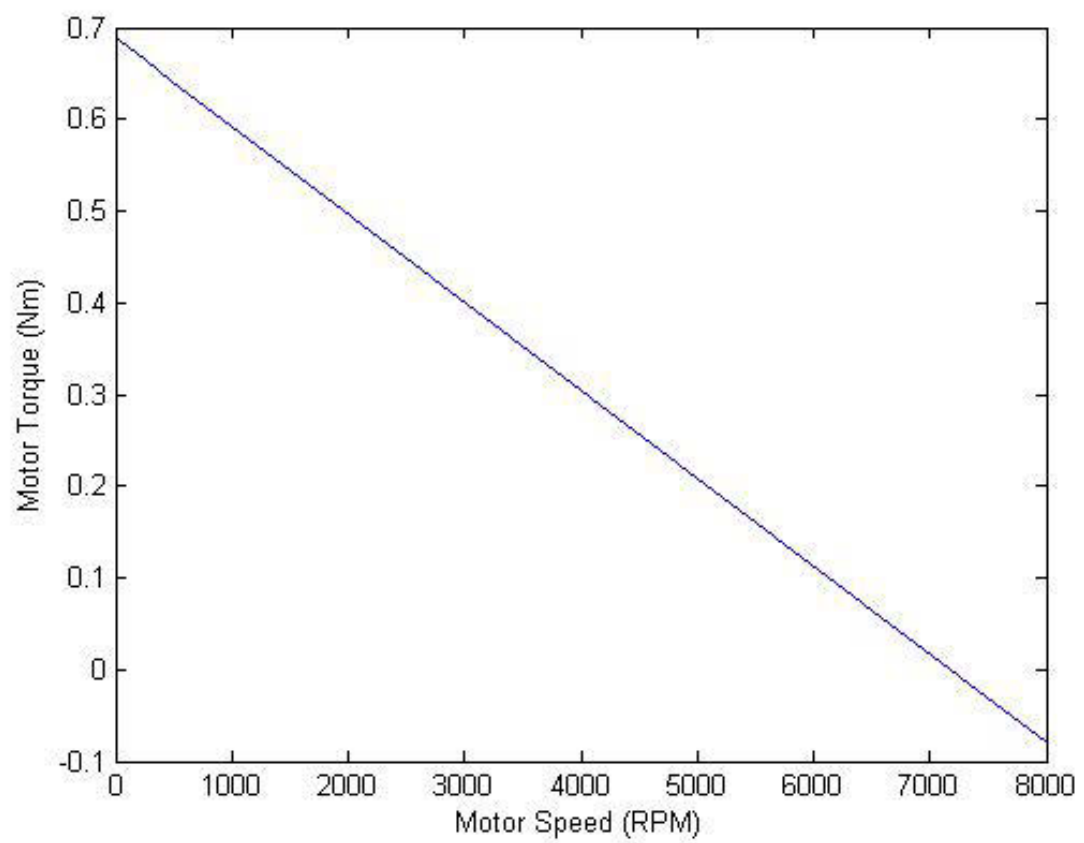

Figure A.11: Motor speed vs available torque for the reaction wheel motor when the supply voltage is capped at $12 \mathrm{~V}$.

\section{A.7 Power System}

The power system is responsible for delivering $5 \mathrm{~V}$ and $12 \mathrm{~V}$ to the various on-board components. A $24 \mathrm{~V}, 10$ Ah battery supplies power, in parallel, to a $5 \mathrm{~V}$ and a 12 $\mathrm{V}$ voltage regulator. The $5 \mathrm{~V}$ regulator has a throughput of $30 \mathrm{~W}$, which is used to power the on-board computers, cameras, and other sensors. The $12 \mathrm{~V}$ regulator can output $350 \mathrm{~W}$ of power, which is used to drive the solenoid valves, reaction wheel, robotic arm, and any future $12 \mathrm{~V}$ components. Push terminals are installed for each voltage level, such that adding a new component requiring power is a trivial task. Cables are run to the upper and lower decks that provide $12 \mathrm{~V}$ and $5 \mathrm{~V}$ of power, so that any component on any deck can access power. The components used in the power subsystem are listed in Table A.8.

Figure A.12 shows the main components how they are installed on the avionics deck of the spacecraft platforms.

A control panel was designed that has a main power switch, as well as status LEDs. An Arduino Mini microcontroller is installed on the control panel and provides some 


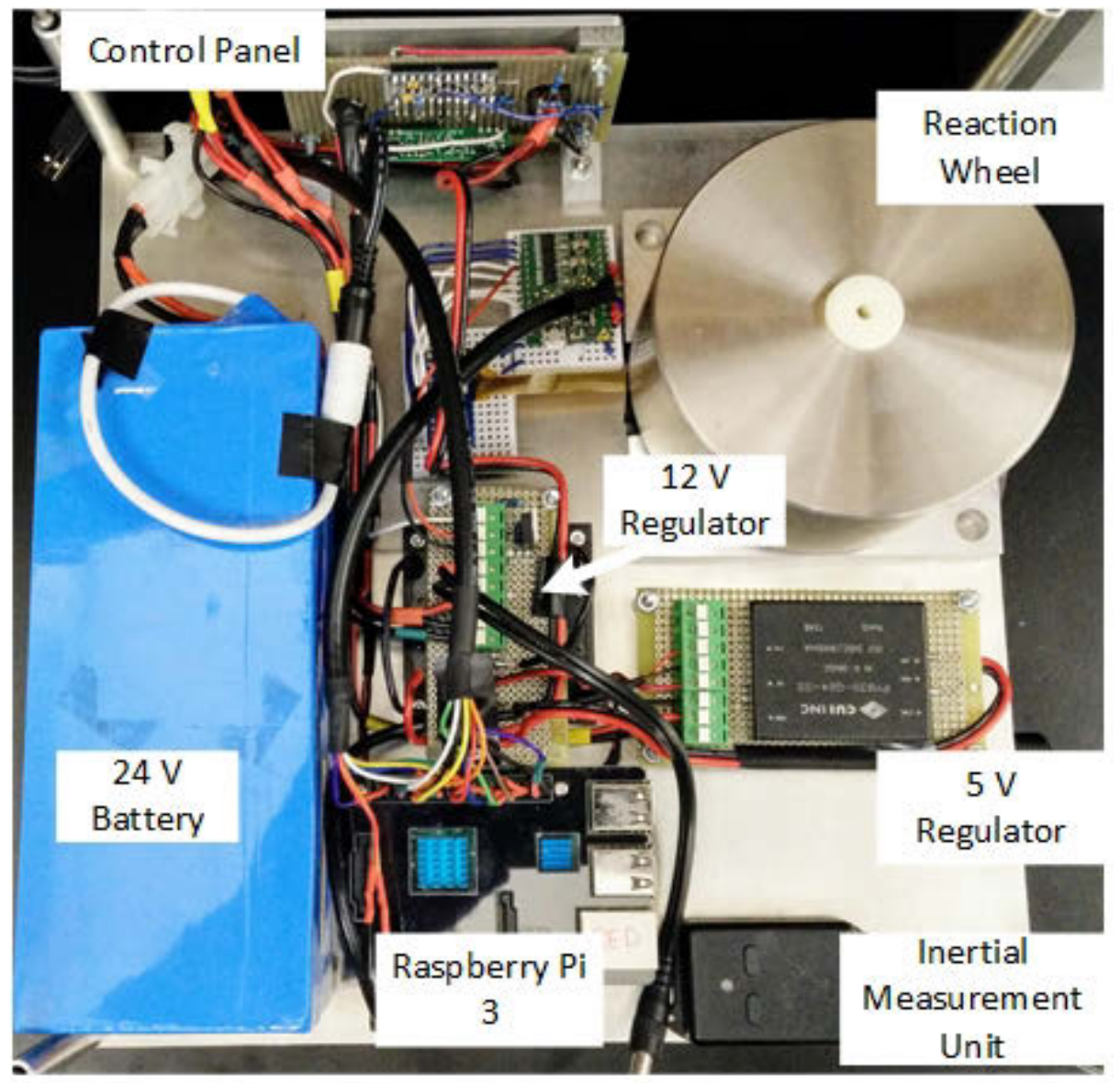

Figure A.12: Avionics deck on board the spacecraft platforms. This deck contains the power subsystem, the on board computer, the reaction wheel, and the control panel. 
Table A.8: Part list for the power system.

\begin{tabular}{|c|c|c|c|}
\hline Name & $\begin{array}{c}\text { Manufacturer } \\
\text { (Supplier) }\end{array}$ & Part Number & Quantity \\
\hline Battery + Charger & $\begin{array}{l}\text { Hui Electronics } \\
\text { (aliexpress.com }\end{array}$ & $\begin{array}{c}\text { BCC-Shenzen-24V- } \\
\text { 10Ah }\end{array}$ & 1 \\
\hline 12 VDC Regulator & CUI Inc (Digikey) & VHB350W-Q24-S12 & 1 \\
\hline 5 VDC Regulator & CUI Inc (Digikey) & PYB30-Q24-S5 & 1 \\
\hline Power Switch & E-Switch (Digikey) & EG1515-ND & 1 \\
\hline Push Terminals & $\begin{array}{l}\text { TE Connectivity } \\
\text { (Digikey) }\end{array}$ & A104596-ND & 20 \\
\hline Arduino Mini & Digikey & 1568-1055-ND & 1 \\
\hline Battery Plug & Digikey & 172-4204-ND & 1 \\
\hline Charging Jack & Digikey & SC1390-ND & 1 \\
\hline $\begin{array}{c}\text { Battery Charging } \\
\text { Plug }\end{array}$ & Digikey & SC1381-ND & 1 \\
\hline
\end{tabular}

basic functionality to the platform. It acts as a battery monitoring computer. It does this by measuring the voltage across a voltage divider on the $24 \mathrm{~V}$ battery line, such that the battery voltage is scaled to a range readable by the Arduino Mini. When the battery voltage drops below $23.8 \mathrm{~V}$, a yellow light on the control panel is illuminated, signaling to the operator that the battery needs to be charged shortly. A charging port is incorporated into the control panel, such that no disassembly is required to charge the battery. If the operator does not charge the battery as it continues to drain, the Arduino Mini will trigger the emergency stop and repeatedly flash the low battery LED when the battery voltage drops below $23.3 \mathrm{~V}$. This is for safety, as when the battery voltage drops too far, the battery circuitry cuts power suddenly. By triggering the emergency stop, all non-critical components are safely stopped. A schematic of the power system is shown in Figure A.13.

The power subsystem is significantly overdesigned, due to the overall platform design changing so rapidly after the power subsystem was purchased. This is convenient for the operator, as the batteries last a very long time between charges. Also, there is a lot of additional capacity for future components to be added. 


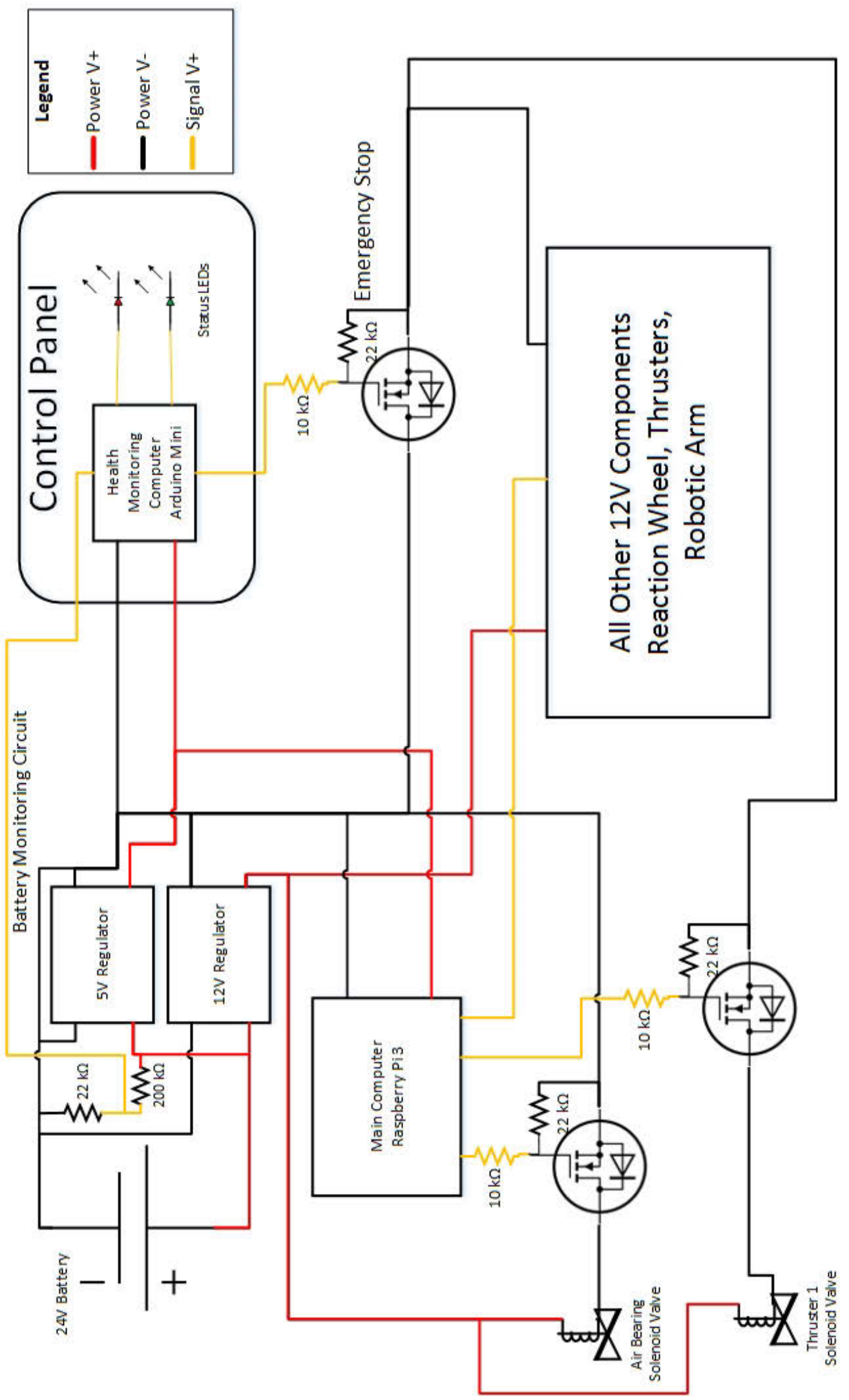

Figure A.13: Electrical schematic present on the spacecraft platforms. 
Table A.9: Part list for the emergency stop system.

\begin{tabular}{cccc}
\hline Name & $\begin{array}{c}\text { Manufacturer } \\
\text { (Supplier) }\end{array}$ & Part Number & Quantity \\
\hline RF Transmitter & Robotshop & RB-Spa-664 & 1 \\
RF Receiver & Robotshop & RB-Spa-662 & 1 \\
E-Stop Button & Amazon & & 1 \\
Arduino Mini & Digikey & $1568-1055-\mathrm{ND}$ & 2 \\
\hline
\end{tabular}

\section{A.8 Emergency Stop System}

In an emergency, it is important to have a system that is completely separate from the system that is failing protect the failing system from itself. In this case, a separate wireless link is made from an emergency stop transmitter circuit on the ground station desk to the on board Arduino Mini. The Arduino Mini is responsible for controlling the emergency stop circuitry. All the components on the $12 \mathrm{~V}$ power line pass through a power MOSFET before returning to Ground, except the air bearings solenoid. The Arduino Mini is powered by the $5 \mathrm{~V}$ voltage regulator, and uses one of its digital output pins to send a High or Low signal to the gate of the MOSFET depending on the desired status of the emergency stop. Since the Arduino Mini is powered by the $5 \mathrm{~V}$ regulator, its Ground is the same as the $5 \mathrm{~V}$ and $12 \mathrm{~V}$ Ground. Therefore, since the source pin of the MOSFET is connected to $12 \mathrm{~V}$ Ground, applying a $+5 \mathrm{~V}$ signal to the gate of the MOSFET will exceed the gate-source threshold voltage and allow current to flow.

Communication between the emergency stop button on the ground station table and the Arduino Mini is accomplished through $315 \mathrm{MHz}$ RF transmitters and receivers. The main wireless link between the ground station computer and the Raspberry Pi 3 is through Wifi. Table A.9 shows the emergency stop components used.

The RF transmitter is connected to an Arduino Mini and is embedded in the Emergency Stop Button located on the ground station table. The RF transmitter is wired to the Arduino Mini exactly as outlined in the documentation. A $12 \mathrm{~V}$ supply line is used, to increase the outgoing signal of the transmitter. An antenna is also used, to increase the range of the signal. The transmitter electronics are plugged 


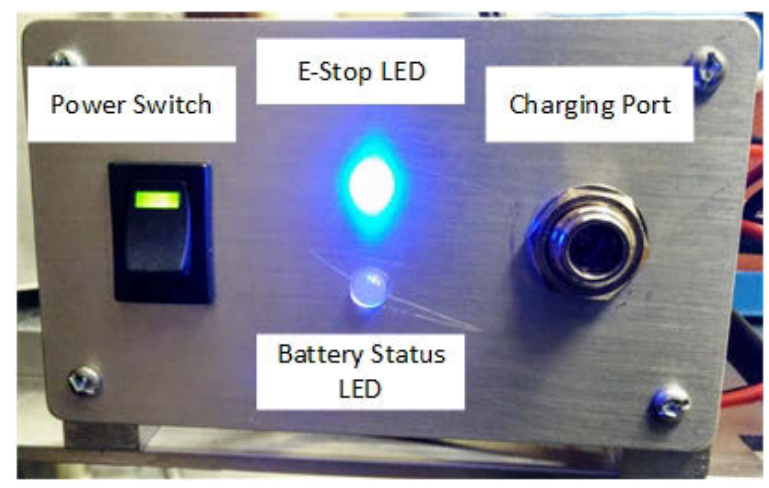

Figure A.14: Control panel on the avionics deck of the spacecraft platform. It includes the main power switch, a charging port, the Emergency Stop LED, and the battery monitoring LED.

into a power source and is continuously operating. The RF receiver electronics are attached to the Arduino Mini on the control panel of the spacecraft platforms exactly as outlined in the RF receiver documentation. An antenna is also used on the receiver to increase reception. When the Emergency Stop button is raised, the RF transmitter repeatedly transmits a "good" signal (the number 20). When the receiver electronics receive the "good" signal, a High signal is applied to the gate of the emergency stop MOSFET. When the Emergency Stop button is pressed, the RF transmitter repeatedly sends a "bad" signal (the number 13). When the "bad" signal is received, a Low signal is sent to the gate of the MOSFET by the Arduino Mini. If no signal is received for 5 seconds, the receiving Arduino Mini automatically triggers the Emergency stop, as there is a fault in the system rendering the system unsafe.

LEDs are used to alert the operator to the current status of the Emergency Stop circuitry. The E-Stop LED is solid blue when the "good" E-Stop signal is being received. When the LED is solid red, this indicates that the "bad" signal was received. When the LED flashes red, this indicates that no E-Stop signal is received, that the E-Stop has been triggered, and that there is a fault in the Emergency Stop circuitry.

The same "good" and "bad" signals are transmitted blindly to all receivers. Therefore, when the E-Stop is triggered, all active spacecraft platforms will simultaneously trigger their Emergency Stop circuitry. The Emergency Stop circuit is shown in Figure A.13, and the exterior of the control panel is shown in Figure A.14. 


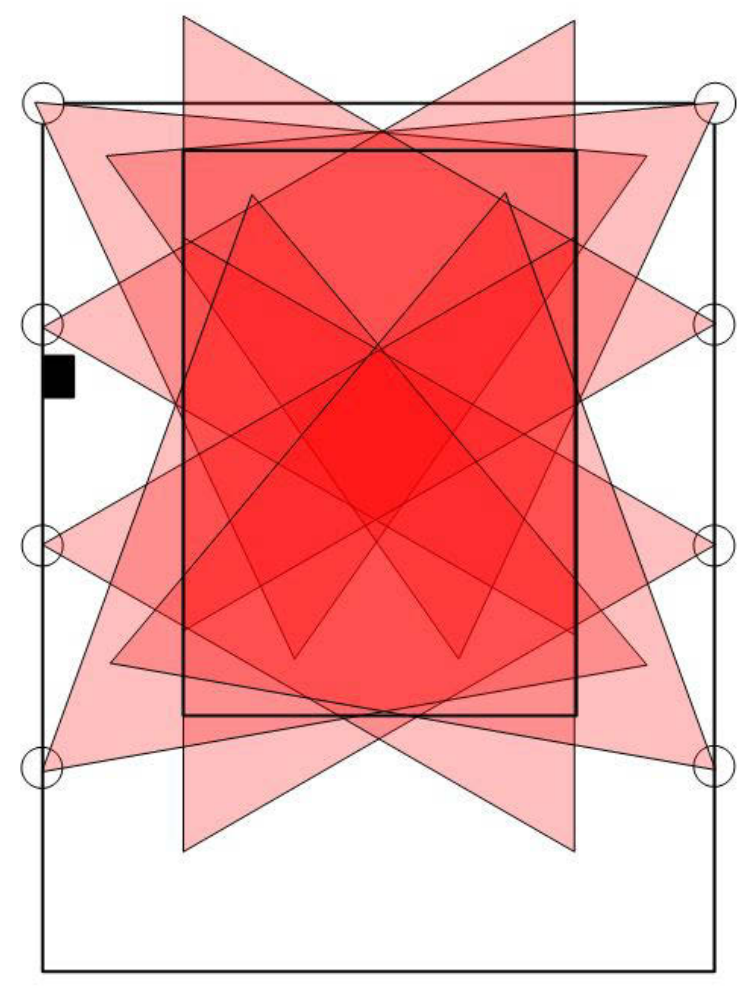

Figure A.15: Camera positioning to ensure even coverage across the table. The black circles represent the cameras, and the red cones represent the field of view of each camera.

\section{A.9 Ground Truth System}

During experiments, ground truth data is required to compare the perceived performance of the spacecraft to its actual performance. For this reason, a PhaseSpace X2E camera system was purchased. It consists of 8 cameras that are positioned around the lab, facing the granite table. All 8 cameras are connected to a provided server that processes the output from the 8 cameras, and returns the resolved position and attitude of the spacecraft platforms, in real time, at $240 \mathrm{~Hz}$. It is important to have multiple cameras covering each section of the table. Therefore, a symmetric camera arrangement was used, shown in Figure A.15. This technique ensures at least three cameras can see all locations on the table.

A picture of the lab is shown in Figure A.16, where the black PhaseSpace cameras are clearly visible.

The camera with the antenna communicates with the Microdrivers (battery packs) 


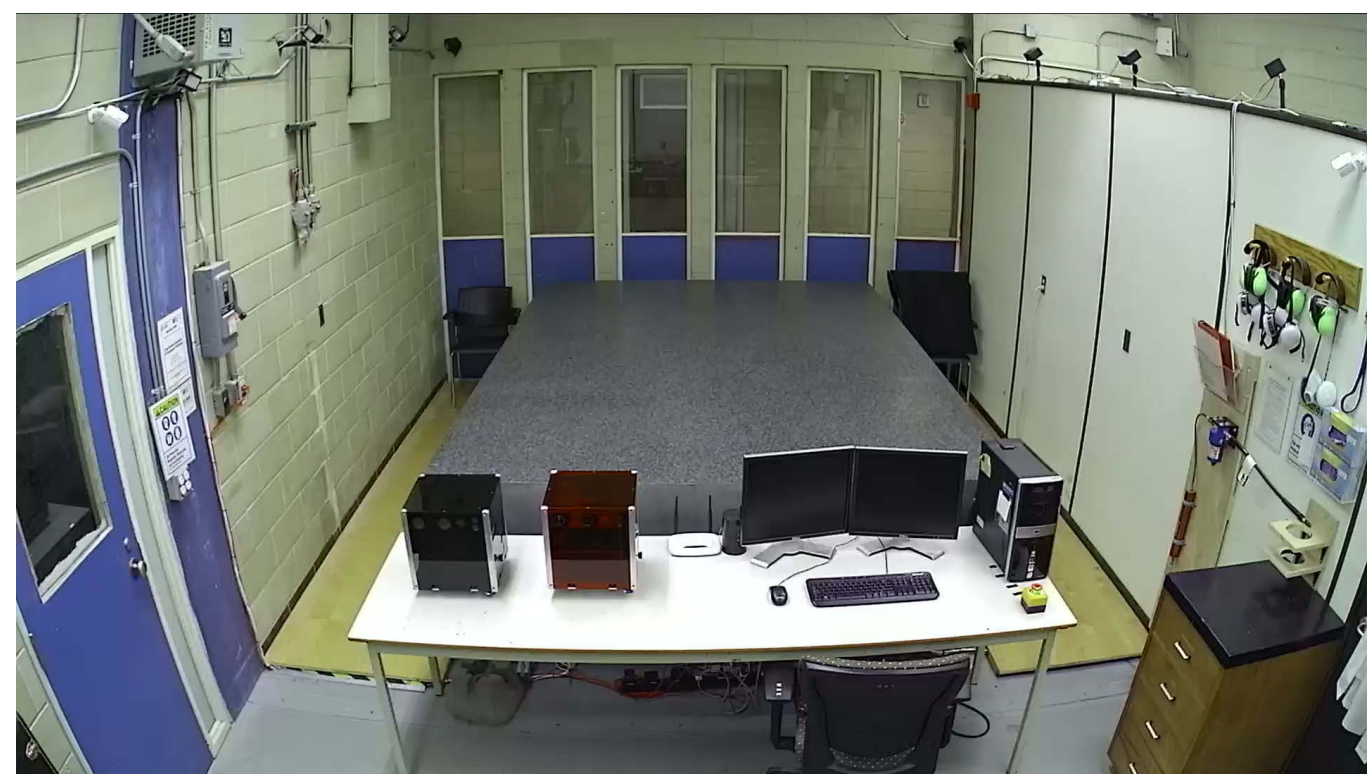

Figure A.16: Overview of the Spacecraft Robotics and Control Laboratory. The black PhaseSpace cameras can be seen surrounding the lab.

that turn on and off the LEDs mounted on the platforms. The Microdrivers, shown in Figure A.17, are turned on by pressing the white button. To turn off a Microdriver, press and hold the white button until the light no longer blinks (about 10 seconds).



Figure A.17: PhaseSpace Microdriver controlling the tracking LEDs.

The microdrivers are assigned to each platform through software. The ID numbers associated with each platform are shown in Table A.10

Any Microdriver can be used for any platform, as long as its ID is set to the correct one for that platform. To set the ID of any Microdriver, follow the steps listed below:

1. Open the PhaseSpace Configuration Manager at 192.168.0.109.

2. Login - user: admin, password: phasespace.

3. Click the "LED Devices" tab. 
Table A.10: Phasespace platform software ID numbers.

\begin{tabular}{cc}
\hline Platform & PhaseSpace ID \\
\hline RED & 1 \\
BLACK & 2 \\
Drone & 3 \\
\hline
\end{tabular}

4. Click "Scan \& Monitor Devices".

5. Click "Scan". Ensure the Microdrivers are turned on. They should be recognized by the software under the Microdrivers heading.

6. The current Microdriver software ID numbers are listed on the right of the screen. The Microdriver with software ID\#1 must be used on the RED platform. Identify the Microdrivers by matching the Hardware ID with the Hardware ID number written on each Microdriver.

7. To change the software ID number of a Microdriver, click "edit IDs" when the desired Microdrivers are connected to the system.

8. Edit the software ID numbers to your preference.

9. Select the check boxes beside the Microdriver Hardware ID and then click "Force Encode Selected" to upload the new software IDs to the Microdrivers.

This way, if a Microdriver fails, another one can easily take its place by simply changing the software ID.

To pair a new Microdriver to the system, follow the above steps to enter "Scan" mode. Then, click and hold the white button on the Microdriver until the LED flashes 3 times. This puts the Microdriver into pairing mode and will be detected by the PhaseSpace system.

Once the cameras are installed and the microdrivers are paired, the system needs to be calibrated. This is done by moving an object with a known size through the field of view of all the cameras. A wand is provided by PhaseSpace and is used to calibrate and align the camera system. 


\section{A.9.1 Calibration}

Calibrating the PhaseSpace system allows the cameras to accurately know their relative positions. When all the cameras agree on the location of the spacecraft, the server can resolve the spacecraft position very precisely and quickly. To calibrate the PhaseSpace system, follow the steps listed below.

1. Locate the calibration wand and the large battery pack (driver).

2. Plug the wand into the battery pack, as shown in Figure A.18. Turn on the battery pack by pressing the white button. If it does not turn on, charge the battery pack using a microUSB cable.

3. Turn on the PhaseSpace server by pressing the black button on the left of the unit.

4. Open Chrome on the ground station computer and navigate to 192.168.0.109. Login with user: admin, pass: phasespace.

5. Click the "OWL Server" tab. Click "Calibration"

6. A popup should appear. Click "Connect" and the cameras and wand should turn on. Set the wand LED brightness to $55 \%$ by dragging the slider. When ready, click "Capture".

7. Move the wand through the volume you would like to be calibrated. Make sure to access all the areas around the table as well. Continue until the boxes are nearly completely full with green. It is okay to leave a few empty boxes.

8. When done, deselect the "Capture" checkbox. Then choose "Exhaustive" as the calibration quality and then click "Calibrate".

9. The system will calibrate and return a success message upon completion. Click SAVE.

The system is now properly calibrated. To test whether the calibration was successful, simply place a platform on the table and log position data. If the standard 


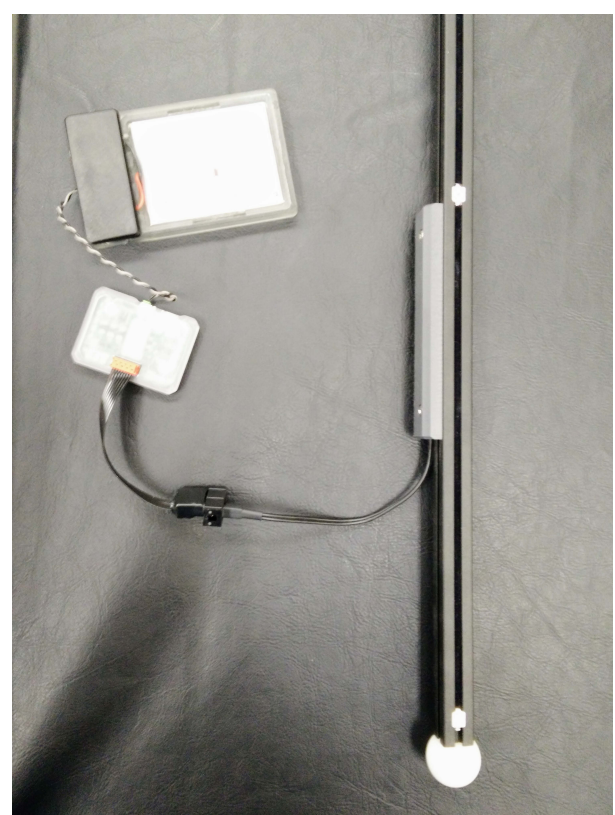

(a) Calibration wand plugged into battery pack

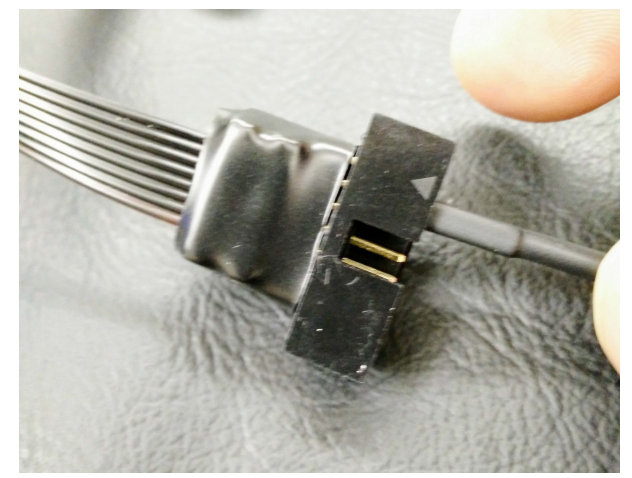

(b) Close up of connection between wand and battery pack

Figure A.18: Wand used for calibrating and aligning the PhaseSpace system.

deviation of the data is low, (i.e., less than $0.1 \mathrm{~mm}$, the calibration was successful). Now, the reference frame of the PhaseSpace system needs to be aligned with the desired reference frame of the table. This is called Alignment, and is presented in the following subsection. The system must be realigned after each calibration.

\section{A.9.2 Alignment}

Aligning the PhaseSpace system is to ensure the XY axes of the PhaseSpace system are aligned with the XY axes of the inertial reference frame chosen in the laboratory. Sitting at the ground station, the origin of the inertial reference frame is at the far left corner of the table. The $+\mathrm{X}$ direction is towards you, along the long edge of the table. The $+\mathrm{Y}$ direction is to the right, along the far short edge of the table. The $+\mathrm{Z}$ axis points upward. When the spacecraft translate on the table, they should only move in the XY plane. If significant translation in the $\mathrm{Z}$ plane are observed, the system must be realigned. Each time the camera system is calibrated, it must be realigned. The procedure for aligning the coordinate system is as follows: 
1. Locate the calibration wand and the large battery pack (driver).

2. Plug the wand into the battery pack, as shown in Figure A.18. Turn on the battery pack by pressing the white button. If it does not turn on, charge the battery pack using a microUSB cable.

3. Turn on the PhaseSpace server by pressing the black button on the left of the unit.

4. Open Chrome on the ground station computer and navigate to 192.168.0.109. Login with user: admin, pass: phasespace.

5. Click the "OWL Server" tab. Click "Alignment"

6. A popup will appear, the cameras will turn on, and the want should illuminate. Set the wand LED brightness to $55 \%$ by dragging the slider.

7. Position the wand above the origin of the table in the vertical direction. The handle of the wand should be near the top. The LEDS should face towards the inside of the table. Have someone click "Snapshot" on the popup to record the first frame of the alignment. The computer screen should flash green if it was successful.

8. Next, position the wand at the far $+\mathrm{X}$ direction of the table. That is, the close left hand corner as viewed from sitting at the ground station. Position the wand in the same fashion as the previous capture. Take another snapshot.

9. Finally, position the wand over the origin once more. However, this time invert the wand, such that the handle is near the bottom of the wand. Align the LEDs towards the centre of the table and take a snapshot. Ensure the wand is as vertical as possible.

10. The system should now be properly aligned. The cameras will look like they are rotated in space on the computer. This is normal, and is due to the way that PhaseSpace defines their coordinate system in their software. 
It is important to test the alignment. Do this by floating a platform and recording data (or having it display on the screen in real time). Float the platform across the table. The $\mathrm{Z}$ position should hover around $300 \mathrm{~mm}$ and not change by more than 100 $\mathrm{mm}$ at any point on the table. If it does, the inertial reference frame is not adequately aligned and must be redone. Make sure to hold the want perfectly vertical during calibration.

\section{A.10 Software}

The software is currently in a redesign. Its details will be included the lab user manual that will be written over the coming year. 